\title{
Headache, coping and quality of life in children
}

Citation for published version (APA):

Bandell-Hoekstra, I. E. (2003). Headache, coping and quality of life in children. [Doctoral Thesis, Maastricht University]. Universiteit Maastricht. https://doi.org/10.26481/dis.20030410ib

Document status and date:

Published: 01/01/2003

DOI:

10.26481/dis.20030410ib

Document Version:

Publisher's PDF, also known as Version of record

\section{Please check the document version of this publication:}

- A submitted manuscript is the version of the article upon submission and before peer-review. There can be important differences between the submitted version and the official published version of record.

People interested in the research are advised to contact the author for the final version of the publication, or visit the DOI to the publisher's website.

- The final author version and the galley proof are versions of the publication after peer review.

- The final published version features the final layout of the paper including the volume, issue and page numbers.

Link to publication

\footnotetext{
General rights rights.

- You may freely distribute the URL identifying the publication in the public portal. please follow below link for the End User Agreement:

www.umlib.nl/taverne-license

Take down policy

If you believe that this document breaches copyright please contact us at:

repository@maastrichtuniversity.nl

providing details and we will investigate your claim.
}

Copyright and moral rights for the publications made accessible in the public portal are retained by the authors and/or other copyright owners and it is a condition of accessing publications that users recognise and abide by the legal requirements associated with these

- Users may download and print one copy of any publication from the public portal for the purpose of private study or research.

- You may not further distribute the material or use it for any profit-making activity or commercial gain

If the publication is distributed under the terms of Article $25 \mathrm{fa}$ of the Dutch Copyright Act, indicated by the "Taverne" license above, 
Headache, coping and quality of life in children 
Headache, coping and quality of life in children.

Inez Bandell-Hoekstra

Thesis Maastricht University

Colofon

Cover: Rens Hoekstra (painting), Piet Bandell (design)

Layout: Inez Bandell

(3) Inez Bandell-Hoekstra, Rosmalen, the Netherlands

ISBN 90-9016673-4

All rights are reserved. No part of this publication may be reproduced or transmitted in any form or by any means, electronical or mechanical, including photocopy recording, or any information storage and retrieval system without permission in writing from the photocopy owner. 


\title{
Headache, coping and quality of life in children
}

\author{
Proefschrift
}

ter verkrijging van de graad van doctor aan de Universiteit Maastricht,

op gezag van de Rector Magnificus, Prof. dr. A.C. Nieuwenhuijzen Kruseman volgens het besluit van het College van Decanen,

in het openbaar te verdedigen

op donderdag 10 april 2003 om 14.00 uur

door

Egberdina Nieskje Gepka Bandell-Hoekstra

geboren op 14 april 1969 te Joure 
Promotores:

Prof. dr. H. Huijer Abu-Saad

Prof. dr. P. Knipschild

Beoordelingscommissie:

Prof. dr. M.A. van den Hout (voorzitter)

Prof. dr. R.J. de Haan (Universiteit van Amsterdam)

Prof. dr. C.M.H. Hosman

Prof. dr. C.P. van Schayck

Dr. M.J. Sorbi (Universiteit Utrecht)

The studies presented in this dissertation were conducted at the Netherlands school of Experimental Psycho Pathology (EPP), acknowledged in 1995 by the Royal Dutch Academy of Science (KNAW).

The studies presented in this dissertation were financed by the Council for Medical and Health Research of the Netherlands (NWO-MW). 
Voor Piet, Gemma en Julia 



\section{CONTENTS}

1 Introduction 9

2 Recurrent headache, coping and quality of life in children: a review 23

3 Prevalence and characteristics of headache in Dutch schoolchildren 47

4 Coping and Quality of life in relation to headache in Dutch 61 schoolchildren

5 The occurrence of recall bias in pediatric headache: a comparison of 73 questionnaire and diary data

6 Effects of a self-help program on headache in children 91

7 Help Yourself cope with headache and stress: effects on quality of life 109 and pain coping in children

8 Conclusion and discussion 129

Summary 145

$\begin{array}{ll}\text { Samenvatting } & 149\end{array}$

Dankwoord/acknowledgement 153

$\begin{array}{ll}\text { Curriculum Vitae } & 157\end{array}$ 

Chapter 1

\section{INTRODUCTION}


10

Chapter 1 


\section{INTRODUCTION}

This first chapter provides a general background on headache, specifies the research questions and presents an overview of the dissertation.

\section{Headache}

Headache is defined as "pain occurring in the head", with the notion that a person has pain whenever he or she says so. 1 Primary headaches are distinguished from secondary headaches. The latter comprise headaches associated with head trauma, vascular disorders, non-vascular intracranial disorders, substances or their withdrawal, noncephalic infections, metabolic disorders, and disorders of the cranium, neck, eyes, ears, nose, sinuses, teeth, mouth or other facial or cranial structures. ${ }^{2}$ In secondary headaches, pain in the head is due to an underlying disorder or a clearly identifiable cause. Primary headaches, however, are idiopathic; each is a disorder in itself. Four types of primary headache can be distinguished: migraine, tension-type headache (TTH), cluster headache and miscellaneous headaches un-associated with structural lesion. The classification committee of the International Headache Society (IHS) has developed operational diagnostic criteria for all headache types. ${ }^{2}$

In children, migraine and $T T H$ represent the two major categories of primary headache. Subtypes of migraine and TTH are presented in table 1.1. The diagnostic criteria of migraine without aura and of episodic TTH are presented in table 1.2. Tables 1.1 and 1.2 present a succinct overview of the main classifications, however, the diagnostic criteria do not fit very well in children. With regard to migraine, some $e^{3,4}$ have suggested that the minimum headache duration be reduced to one hour and to accept bi-frontal as well as bi-temporal location in addition to unilateral location, and photo- or phonophobia, instead of requiring both. Others suggest to let severe headache associated with nausea fit the migraine diagnosis, whether or not the criteria of lacation, quality, and aggravation by physical activities are fulfilled. ${ }^{5}$ No such adaptations have been proposed for TTH, but TTH has hardly been studied in children so far.

It is also important to understand that the IHS presents a classification system for headaches, not patients. ${ }^{6}$ The aim is to classify discrete attacks. One person can suffer from different, co-existing types of headache. All different headache types within one person should be diagnosed and the number of attacks per year should be recorded. 
Table 1.1 Classification of migraine and tension-type headache

\section{Migroine}

1.1 Migraine without aura

1.2 Migraine with aura

1.2.1 migraine with typical aura

1.2.2 migraine with prolonged aura

1.2.3 familial hemiplegic migraine

1.2.4 basilar migraine

1.2 .5 migraine aura without headache

1.2.6 migraine with acute onset aura

1.3 Ophthalmoplegic migraine.

1.4 Retinal migraine

1.5 Childhood periodic syndromes that may be precursors to or associated with migraine

1.5.1 benign paroxysmal vertigo of childhood

1.5.2 alternating hemiplegia of childhood

1.6 Complicatians of migraine

1.6.1 status migrainosus

1.6.2 migrainous infarction

1.7 Migrainous disorder not fulfilling above criteria
Tension-type heodache

1.8 Episodic tension-type headache

1.8.1 episodic tension-type headache associated with disorder of pericranial muscles

1.8.2 episodic tension-type headache unassociated with disorder of pericranial muscles

1.9 Chronic tension-type headache

1.9.1 chronic tension-type headache associated with disorder of pericranial muscles

1.9.2 chronic tension-type headache. unassociated with disorder of pericranial muscles

1.10 Headache of the tension-type not fulfilling above criteria

Source: Headache Classification Committee for the International Headache Society (1988)? 
Table 1.2 Operational diagnostic criteria of migraine without aura and episodic tension-type headache

Migraine without aura (1.1)

A. at least 5 attacks fulfilling B-O

B. Headache ottacks lasting 4-72 hours (untreated or successfully treated)

c. Headache has at least two of the following characteristics:

1. unillateral location

2. pulsating quality

3. moderate or severe intensity (inhibits or prohibits daily activities

4. aggravation by walking stairs or similar routine physical activity

D. During headache at least one of the following:

1. nousea and/or vomiting

2. photophobia and phonophobia

E. At least one of the following:

1. histary and physical and neurological examinations do not suggest one of the disorders listed in groups 5 11 (organic disorder) $^{2}$

2. history and/or physical and/or neurological examinations do suggest such disorder, but is ruled out by appropriate investigations

3. such disorder is present, but migraine attacks do not occur for the first time in close temporal relation to the disorder
Episodic tension-type headache (2.1)

A. At least 10 previous headache episodes fulfilling criteria B-D listed below. Number of days with such headache * $180 /$ year ( $* 15 /$ month).

B. Headache lasting from 30 minutes to 7 days

c. At least two of the following pain characteristics:

1. pressing/tightening (nonpulsating) quality

2. mild or moderate intensity (may inhibit, but does not prohibit activities)

3. bilateral location

4. no aggravation by walking stairs or similar routine physical activity

D. both of the following:

1. no nausea or vomiting (anorexia may occur)

2. photophobia and phonophobia are absent, or one but not the other is present

E. At least one of the following:

1. history, physical and neurological examinations do not suggest one of the disorders listed in groups 5-11 (organic disorder)

2. history and/or physical and/or neurological examinations do suggest such disorder, but is ruled out by appropriate investigations

3. such disorder is present, but tension-type headache does not occur for the first time in close temporal relation to the disorder

In children below age 15, attacks may last 2-48 hours. If the patient falls asleep and wakes up without migraine, duration of attack is until time of awakening.

${ }^{2}$ Groups 5-11 represent the secondary headaches.

Source: Headache Classification Committee for the International Headache Saciety (1988). ${ }^{*}$ 
Despite the foregoing classification, not all headache episodes can be diagnosed. Atypical episodes exist because of early treatment or lack of accurate recall of symptoms. 6

Another limitation is that the complex algorithm of criteria (see table 1.2) complicates headache assessment and diagnosis. This is especially true in a nonclinical situation using self-report.

Notwithstanding the importance of the classification, we took these issues into consideration and decided to focus our study on primary headache in general, without aiming at diagnosing the headache as migraine or TTH.

\section{Headache as a complex entity}

Despite the simplicity of defining headache as "pain in the head", close examination suggests that headache is a very complex disorder. Any attempt to understand headache requires consideration of a range of factors, including the pain, triggers, impact on the individual, treatment and culture. For example, in regard to the pain, it is important to consider the frequency, intensity, duration, location and quality, accompanying symptoms such as nausea and photophobia, aggravating factors, and warning signals, premonitory symptoms or precipitating factors. Sometimes, causative or predisposing factors, or triggers can be identified. The individual's headache response, at physiological, behavioral and cognitive levels, the natural course of headache over time and the headache impact on daily activities or quality of life are important factors. Headache management, including pharmacological and non-pharmacological strategies and pain and stress coping, and their previous success or failure, add to the complexity of headache as a discrete entity. In addition to the range of factors identified above, the accumulation of all prior features with regard to past headache and pain experiences, that forms the pain beliefs and expectancies with regard to headache experience and management, are factors that shape the individual's pain experience. Finally, an understanding of the complexity of headache requires consideration of internal psychological modulators, such as personality, anxiety and depression, and external influences, such as pain models and reinforcement of pain behavior within the culture, family and peer group. In an attempt to simplify the complex phenomenon of headache, we have focused on the coping and Quality of Life aspects of headache disorder. A literature review and a conceptual model on headache, coping and QoL is presented in chapter two. 


\section{Headache as a public health problem}

The experience of an occasional headache is common to almost all people, including children and adolescents. A lifetime prevalence of over $90 \%$ is reported in the general population aged 12 years and up. ${ }^{7-9}$ Rasmussen ${ }^{10}$ reported that $20 \%$ of the general adult population experiences a headache during a period of 2 weeks. Weekly headaches are reported by $6-29 \%$ of children in the general population (see chapter three). Differences in reparted prevalence rates may be due to differences in headache definition and study design. Population-based studies often rely on retrospective self-report: subjects are asked to think back and report on their headache complaints over the past month or year. Recall errors may be a methodological problem in these studies (see chapter five).

Headache prevalence is poorly reflected in health-care utilization rates. ${ }^{11}$ Approximately $70 \%$ of headache sufferers never seek medical help for their pain; nor do $50 \%$ of subjects with migraine and $80 \%$ of subjects with tensiontype headache. ${ }^{12-15}$ Only $2 \%$ of all headache episodes in children are presented to the general practitioner (GP). ${ }^{16}$ Only $18 \%$ of children with headaches that present to the GP are diagnosed with primary headaches. The incidence of primary headache in 5-9 and 10-14 year old children, within general practice in the Netherlands, is 7 and 13 per 1000 person years, respectively. ${ }^{17}$

Long-term follow-up studies on the incidence of headache in the general population, and on the natural course of headache, are scarce. A prospective study conducted by Bille ${ }^{18}$ showed that $60 \%$ of children with migraine continue to experience migraine after the age of 30 years. A large birth cohort study in the UK showed an increased risk of headache at age 33 for children with frequent headaches (Odds ratio of 2.2). ${ }^{19}$ A small cohort study on 3-10 year old children with headache at an outpatient clinic in the Netherlands, showed that $94 \%$ of the children with migraine continued to experience headaches after 4 years, and $54 \%$ of the children with other headache types (TTH, post-traumatic and atypical headache). ${ }^{20}$

Based on a comparison of two similar studies in 1974 and 1992. Sillanpää and Anttila ${ }^{21}$ have hypothesized an increase in the prevalence of headache in children. Our replication of the 1983 cross-sectional study on headache in Dutch school children ${ }^{22}$, enabled us to test this hypothesis (chapter three).

The prevalence, burden on the individual and society, and large proportion of individuals engaged in self-treatment depict headache as a significant and serious public health problem. The societal costs include directly measurable costs associated with medical care, indirect costs from loss of work 
productivity, and less tangible costs related to QoL. ${ }^{23}$ The definition and assessment of societal costs are subject to discussion, and perhaps impossible to describe for children. ${ }^{24}$ The individual burden of headache can be considered, and described, in terms of how the child has to cope with the pain and the impact of the disorder on the child's QoL. An elaboration on the concepts of coping and QoL will be presented in chapter two. The description of pain coping and QoL within groups of school children with a low, medium and high headache severity can be found in chapter four.

\section{Headache management in the general population}

Most people deal with their headaches themselves, rather than seeking medical attention. In the literature, self-management is often restricted to "using overthe-counter medication". This approach limits headache management to pain management, whereas, the recognition and management of triggers is important. Moreover, non-pharmacological treatments should be considered, especially in children, because of their reluctance to use (prophylactic) medication. 25,26

Controlled studies on the efficacy of non-pharmacological treatments in children all involve relaxation (table 1.3). The aim of the relaxation exercises, with or without biofeedback, additional medication or cognitive training, is not always clearly stated, but in most studies, the focus is on the management of stress, not pain. Psychological or mental stress is most frequently mentioned as a headache trigger, by adults ${ }^{27-29}$ and children. $24,28,30,31$ A plausible physiological explanation of the role of stress in migraine has recently been given. ${ }^{32}$ In TTH the physiological role of stress has often been explained by muscle contraction or ischemia, but scientific support is minimal due to a lack of studies. ${ }^{27}$

The role of stress in primary headaches starts early on when children experience their first headache attacks, and based on the results of descriptive and intervention studies, is hypothesized to be a causative factor. Some children are unable to comfortably control a common, yet stressful situation, within their family, peer-group, school or sports. They become increasingly anxious about it and this may provoke a headache attack. The headache may, in this context, provide an avenue for escape. That is, because of the pain, the child is removed from the stressful situation. Attention is paid to the headache, but often nat to the source of stress, so anxiety in similar situations will trigger additional attacks. Persisting headaches will evoke other factors, and a complex disorder can take shape. ${ }^{33}$ In addition to stress as a headache trigger, chronic or 
recurrent headache can be viewed as a source of stress in itself, that serves to exacerbate or maintain the pain. ${ }^{34}$

The importance of stress in children's headaches warrants a treatment that is focused on education about stress and stress-management techniques and incorporation of these techniques into pain management. Children must learn to recognize signs of body tension, to identify stressors, and develop ways of reducing stress, such as relaxation strategies.

Stress is defined as external or internal demands (and conflicts between them) that are taxing or exceeding the resources of the person. ${ }^{35}$ Coping can be defined as intentional cognitive and behavioral efforts to manage stress. ${ }^{36}$ Cognitive appraisal is the link between stress and coping; it is the conscious or unconscious process of categorizing an encounter "and its various facets, with respect to its significance for well-being. Primary appraisal concerns the question "Am I in trouble or not?", and secondary appraisal "What, if anything. can I do and what are the consequences?". In addition to relaxation strategies, the treatment must incorporate education on the role of cognitions (i.e., negative thoughts) and behavior, in order to support problem identification and to broaden the range of coping strategies.

Only two studies on headache in children so far (see table 1.3), have been published that evaluate a treatment program that is comprised of both relaxation and cognitive training. Osterhaus et al. ${ }^{37}$ conducted a study on the effect of a combination of relaxation, biofeedback and cognitive training, presented in the school setting. They found a positive treatment effect on headache frequency and duration, but not on pain intensity. McGrath et al. ${ }^{26}$ studied the effect of a program that combined relaxation and cognitive training, comparing a therapist-administered version presented in a clinical setting and a self-administered version at home. Both treatment versions were equally effective and showed more headache improvement than a placebo control group. Because of the reduced amount of therapist involvement, the "Help Yourself" training program was more cost-efficient. Since self-help programs are less threatening and more easily accessible, they are suitable for a large number of potential consumers. ${ }^{46}$

The prevalence of frequent primary headaches in the general population of children and adolescents emphasizes the need for preventive measures. Enhancing self-management skills and the prevention of headache chronicity have become major goals. We therefore designed a randomized trial to test the effectiveness of the "Help Yourself" training in Dutch school children who experienced frequent headaches (see chapters six and seven). 
Table 1.3 Randomized trials on non-pharmacological headache treatments (other than biafeedback-ionly) in children

\begin{tabular}{|c|c|c|c|c|}
\hline Article & Age & $m$ & Headache type & Groups \\
\hline $\begin{array}{l}\text { Engel and Rapoff, } \\
1990^{38}\end{array}$ & $11-21$ & $?$ & $\begin{array}{l}\text { non-malignant } \\
\text { frequent headoche } \\
(\mathrm{z} 3 / \text { month })\end{array}$ & $\begin{array}{l}\text { 1. autogenic relaxation } \\
\text { 2. progressive relaxation } \\
\text { 3. autogenic plus progressive relaxation } \\
\text { 4. waiting-list control group }\end{array}$ \\
\hline $\begin{array}{l}\text { Engel ett al. } \\
1992^{39}\end{array}$ & $11-21$ & 17 & $\begin{array}{l}\text { non-malignant } \\
\text { frequent headache } \\
(\mathrm{z} 3 / \text { month) }\end{array}$ & $\begin{array}{l}\text { 1. outogenic relaxation } \\
\text { 2. progressive relaxation } \\
\text { 3. autogenic plus progressive relaxation } \\
\text { 4. waiting-list controll group }\end{array}$ \\
\hline $\begin{array}{l}\text { Fentress et al. } \\
1986^{40}\end{array}$ & $8-12$ & 18 & migraine & $\begin{array}{l}\text { 1. biofeedback + relaxation-response }(n=6) \\
\text { 2. relaxation-respanse }(n=6) \\
\text { 3. waiting-list control group }(n=6)\end{array}$ \\
\hline $\begin{array}{l}\text { Fichtel \& } \\
\text { Larsson, } 2001^{41}\end{array}$ & $13-18$ & 36 & $\begin{array}{l}\text { migraine anly or with } \\
\text { tension-type }\end{array}$ & $\begin{array}{l}\text { 1. relaxation }(n=20) \\
\text { 2. waiting-list control group }(n=16)\end{array}$ \\
\hline Labbé, $1995^{42}$ & $7-18$ & 30 & migraine & $\begin{array}{l}\text { 1. biafeedback + autogenic relaxation }(n=) \\
\text { 2. autogenic relaxation only }(n=) \\
\text { 3. waiting-list control group }(n=)\end{array}$ \\
\hline $\begin{array}{l}\text { Larsson \& Melin, } \\
1986^{43}\end{array}$ & $16-18$ & 31 & $\begin{array}{l}\text { frequent headache, } \\
\text { tension or mixed }\end{array}$ & $\begin{array}{l}\text { 1. relaxation training }(n=11) \\
\text { 2. information-contact }(n=13) \\
\text { 3. self-registration only }(n=7)\end{array}$ \\
\hline $\begin{array}{l}\text { Larsson et al } \\
1987^{44}\end{array}$ & $16-18$ & 41 & $\begin{array}{l}\text { chronic headache, } \\
\text { migraine, tension or } \\
\text { mixed }\end{array}$ & $\begin{array}{l}\text { 1. relaxation, therapist/groups/school }(n=14) \\
\text { 2. self-help relaxation at home }(n=16) \\
\text { 3. untreated self-monitoring group }(n=11)\end{array}$ \\
\hline $\begin{array}{l}\text { Larsson et al } \\
1987^{45}\end{array}$ & $16-18$ & 34 & $\begin{array}{l}\text { Tension only or mixed } \\
\text { with migraine }\end{array}$ & $\begin{array}{l}\text { 1. self-help relaxation }(n=?) \\
\text { 2. problem discussion }(n=?) \\
\text { 3. self-registration }(n=?)\end{array}$ \\
\hline $\begin{array}{l}\text { Larsson et al. } \\
1990^{46}\end{array}$ & $16-18$ & 43 & tension headeche & $\begin{array}{l}\text { 1. self-help relaxation ( } n=31) \\
\text { 2. waiting-list control group }(n=17) \\
\text { Then: cross-over of } \\
\text { 1. self-help relaxation + a muscle relaxant } \\
\text { 2. self-help relaxation + placebo medication }\end{array}$ \\
\hline $\begin{array}{l}\text { McGrath et al., } \\
1988^{47}\end{array}$ & $9-17$ & 99 & migraine & $\begin{array}{l}\text { 1. relaxation }(n=32) \\
\text { 2. psychalogical placebo }(n=30) \\
\text { 3. own best effort }(n=37)\end{array}$ \\
\hline $\begin{array}{l}\text { McGrath et al. } \\
1992^{26}\end{array}$ & $11-18$ & 73 & migraine & $\begin{array}{l}\text { 1. Help Yourself, therapist, climic }(n=23) \\
\text { 2. Help Yourself, self, at home }(n=24) \\
\text { 3. plecebo-control group }(n=26)\end{array}$ \\
\hline $\begin{array}{l}\text { Olness et all. } \\
1987^{4}\end{array}$ & $6-12$ & 28 & migraine & $\begin{array}{l}\text { 1. placebo - propranolol - self-hypnosis }(n=) \\
\text { 2. propranolol - placebo - self-hypnosis }(n=)\end{array}$ \\
\hline $\begin{array}{l}\text { Oliness et al. } \\
1999^{32}\end{array}$ & $5-12$ & 28 & migraine & $\begin{array}{l}\text { 1. no intervention }(n=14) \\
\text { 2. self-regulation }(n=14)\end{array}$ \\
\hline $\begin{array}{l}\text { Osterthous et al. } \\
1993^{37}\end{array}$ & $12-19$ & 41 & migraine & $\begin{array}{l}\text { 1. experimental group ( } n=32) \\
\text { 2. waiting-list control group }(n=9)\end{array}$ \\
\hline $\begin{array}{l}\text { Passchier et al. } \\
1990^{4 \$}\end{array}$ & $12-18 ?$ & 271 & weekly headaches & $\begin{array}{l}\text { 1. progressive relaxation training } \\
\text { 2. placebo control group }\end{array}$ \\
\hline $\begin{array}{l}\text { Richter et al., } \\
1986^{50}\end{array}$ & $9-18$ & 42 & migraine & $\begin{array}{l}\text { 1. relaxation training }(n=15) \\
\text { 2. cognitive coping }(n=15) \\
\text { 3. placebo control group }(n=1.2)\end{array}$ \\
\hline
\end{tabular}




\section{Study design}

The purpose of our own research was 1) to gain insight in headache prevalence and in headache-related pain coping and Quality of Life in the general population of school-aged children, and 2) to test the short- and long-term effect of a cognitive-behavioral training program focused on teaching self-management skills to children with frequent headaches.

The research consisted of two studies: a cross-sectional study and a randomized trial. The study sample in the cross-sectional study served as the study population for the trial.

The following research questions were addressed.

1) What is the prevalence of headache in school children in the Netherlands?

a. Are these prevalence figures higher than previous findings in 1983?

b. Do children accurately recall prior headache complaints?

2) Do children with a low, medium or high headache severity differ with regard to headache characteristics, use of pain coping strategies and Quality of Life?

3) Is the "Help Yourself" training program more effective on headache, pain coping and QoL than a control training, in children of the general population with frequent primary headaches?

Focus of the study is on children in grades 6,7 and 8 of elementary school (aged 9 to 12 years) and grades 1 to 4 of high school (aged 12 to 17 years).

\section{Overview of the dissertation}

This dissertation is organized as follows.

Chapter 2 presents a literature review and a conceptual model on headache, pain coping, and QoL in children. Together, chapters 1 and 2 constitute the framework of the study.

Chapters 3, 4 and 5 present the results of the cross-sectional study. Chapter 3 is limited to the description of headache characteristics. Chapter 4 completes the picture with results on pain coping and QoL. Chapter 5 is devoted to a methodological problem, namely 'recall errors' encountered in retrospective cross-sectional studies with self-report questionnaires on the presence of symptoms or diseases. 
Chapters 6 and 7 present the results of the randomized trial on the effectiveness of Help Yourself, again distinguishing the results on headache (chapter 6) and on pain coping and QoL (chapter 7).

Chapter 8 concludes on the major findings of the study, reflects on the study methodology and gives recommendations with regard to clinical practice and future research.

\section{REFERENCES}

1. McCaffery M. Nursing management of the patient with pain. Philadelphia: Lippincott, 1979.

2. IHS: Headache classification committee of the International Headache Society. Classification and diagnostic criteria for headache disorders, cranial neuralgias and facial pain. Cephalalgia 1988;8 (suppl. 7);1-96.

3. Abu-Arafeh I. Russell $G$. Prevalence of headache and migraine in schoolchildren. $B M J$ 1994:309:765-768.

4. Winner $P$. Wasiewskil W, Gladstein J, Linder 5 . Multicenter prospective evaluation of proposed pediatric migraine revisions to the IHS criteria. Pediatric Headache Committee of the Americon Association for the Study of Headache. Headache 1997:37:545-548.

5. Wober-Bingol $C$, Wober $C$, Wagner-Ennsgraber $C$, Karwautz $A$, Vesely $C$, Zebenhoizer $K_{*}$ Geldner J. IHS criteria for migraine and tension-type headache in children and adollescents. Headache 1996:36:231-238.

6. Olesen J. Classification. In: Olesen J. Tfelt-Hansen. Welch KMA. The Headaches. New York: Raven Press, 1993, pp 9-14.

7. Linet MS, Stewart WF, Celentano DD, Ziegler D, Sprecher M. An epidemiologic study of headache among adolescents and young adults. J,AM,A 1989:261:2211-2216.

8. Nikiforow R. Hokkanen E. An epidemiological study of headache in an urban and a rural population in northern Finland. Headache 1978:18:137-145.

9. Rasmussen BK, Jensen R, Olesen J. Questionnaire versus clinical interview in the diagnosis of headache. Headache 1991:31:290-295.

10. Rasmussen BK. Epidemiology. In: Olesen J. Tfelt-Hansen P. Welch KMA. The Headaches. New York: Raven Press. 1993, pp. 15-20.

11. Lipton RB, Stewart WF. Elentano DD, Reed ML. Undiagnosed migraine: a comparison of symptom-based and physician diagnosis. Archives of Internal Medicine 1992;152:1273-1278.

12. MacGregor, EA (1998). Managing migraine in primary care. Blackwell Science, 1998, 272 pp.

13. Mortimer MJ, Kay J, Jaron A. Epidemiollogy of theadache and childhood migraine in an urban general practice using Ad Hoc, Vahlquist and IHS criteria. Dev Med Child Neurol. 1992;34:1095-1101.

14. Rasmussen BK, Jensen $R$, Olesen $J$. Impact of headache on sickness absence and utilisation of medical services. Danish population study. Journal of Epidemialogy and Community Health 1992:46:443-446.

15. Waters WE, O'Connor PJ. Epidemiology of headache and migraine in women. Journal of Neurology, Neurosurgery and Psychiatry 1971;34:148-153. 
16. Bruijnzeels MA, Foets $M$, van der Wouden JC, wan den Heuvel WJA, Prins A. Everyday symptoms in childhood: accurrence and GP consultation rates. British Journal of General Practice 1998:48:880-885.

17. Van der Wouden JC, van der Pas P, Bruijnzeels MA, Brienen JA, van Suijlekom-Smit LWA. Headache in children in Dutch general practice. Cephalalgia 1999:19:147-150.

18. Bille B. Migraine in childhood and its prognosis. Cephalalgia 1981:1:71-75.

19. Fearon $P$. Hotopf $M$. Relation between headache in childhood and physical and psychiatric symptoms in adulthood: national birth cohort study, BMJ 2001;322:1-6.

20. Versluis R, Brouwer OF. Hoofdpijn bij kinderen: een follow-up onderzoek. Tijdschrifti voor Kindergeneeskunde 1995;63:141-143.

21. Sillanpäa $M$. Anttila P. Increasing prevalence of headache in 7-year-old schoolchildrem. Headache 1996:36:466-470.

22. Passchier J, Orlebeke JF. Headaches and stress in schoolchildren: an epidemiological study. Cephalalgia 1985:5:167-176.

23. Stewart WF, Lipton RB. Background to the headaches. Sacietal impact of headache. In: Olesen J, Tfelt-Hansen, Welch KMA. The Headaches. New York: Raven Press, 1993, pp 2934.

24. McGrath PA. Headache in children: the nature of the problem. In: McGrath PA, Hillier LM. The child with headache: diagnosis and treatment. Progress in pain research and management. Seattle: IASP Press, 2001 (19), pp. 1-27.

25. Lascelles MA, McGrath PJ, Sullivan MJL, Werk A. Self-administered treatments for adolescents with headache: description, applications, and limitations. Headache Quarterly 1991:2:196-200.

26. McGrath PJ, Humpgreys $P$, Keene $D$, Goodman JT, Lascelles MA, Cunningham SJ, Firestone $P$. The efficacy and efficiency of a self-administered treatment for adolescent migraine. Pain 1992:49:321-324.

27. Andrasik F. Passchier J. Tension-type headache, cluster headache, and miscellaneous headaches. Psychological aspects. In: Olesen J, Tfelt-Hansen, Welch KMA. The Headaches. New York: Raven Press, 1993، pp 489-492.

28. Passchier J, Andrasik F. Migraine. Psychological factors. In: Olesen J, Tfelt-Hansen, Welch KMA. The Headaches. New York: Raven Press, 1993, pp 233-240.

29. Peatfield RC, Olesen J. Migraine. Precipitating factors. In: Olesen J, Tfelt-Hansem, Welch KMA. The Headaches. New York: Raven Press, 1993, pp 241-245.

30. Egermark-Erilksson I. Prevalence of headache in Swedish schoolchildren, Acta Paediatr Scand 1982;71:135-140.

31. Maratos J. Wilkinson M. Migraine in childreni a medical and psychiatric studly. Cephalalgia. 1982:2:179-187.

32. Olness K, Hall H, Rozniecki JJ, Schmidt W. Theoharides TC. Mast cell activation in children with migraine before and after training in self-regulation. Headache 1999:39:101-107.

33. McGrath PA, Hillier LM. The child with headache: diagnosis and treatment. Seattle: IASP Press, 2001.

34. Andrasik F, Gerber W-D. Relaxation, biofeedback, and stress-coping therapies. In: Olesen J, Tfelt-Hansen, Welch KMA. The Headaches. New York: Raven Press, 1993, pp 833-841.

35. Lazarus RS. Emotion and Adaptation. New York: Oxford University Press: 1991.

36. Lazarus RS. Folkman S. Stress, Appraisal and Caping. New York: Springer: 1984.

37. Osterhaus SO, Passchier $\mathrm{J}$, wan der Helm-Hylkema $H$, de Jong KT, Orlebeke JF, de Grauw A.J. Dekker PH. Effects of behavioral psychophysiological treatment on schaalchildren with 
migraine in a nonclinical setting: predictors and process variables. I Pediatr Psychal 1993:18:697-715.

38. Engel JM, Rapoff MA. Biofeedback-assisted relaxation training for adult and pediatric headache disorders. Occupational Therapy Journal of Research 1990:10:283-299.

39. Engel JM, Rapoff MA, Pressman AR. Lang-term follow-up of relaxation training for pediatric headache disorders. Headache 1992:32:152-156.

40. Fentress DW, Masek BJ, Mehegan JE, Benson H. Biafeedback and relaxation-response training in the treatment of pediatric migraine. Developmental Medicine and Child Neuriology 1986:28:139-146.

41. Fichtel $\AA$, Larsson $B$. Does relaxation treatment have differential effects on migraine and tension-type headache in adolescents? Headache 2001:41:290-296.

42. Labbé EE. Treatment of childhood migraine with autogenic training and skin temperature biofeedback: a component analysis. Headache 1995;35:10-13.

43. Larsson B. Melin L. Chronic headaches in adalescents: treatment in a school setting with relaxation training as compared with information-contact and self-registration. Pain $1986: 25: 325-336$.

44. Larsson B, Daleflod B, Hakansson L. Melin L. Therapist assisted versus self-help relaxation treatment of chronic headaches in adolescents: a school-based intervention. Journal of Child Psychology, Psychiatry and Allied Disciplines 1987:28:127-136.

45. Larsson B. Melin L. Lamminen M. Ullisted F. A school-based treatment of chronic headaches in adolescents. Journal of Pediatric Psychalogy 1987;12:553-566.

46. Larsson B, Melin L. Doberl A. Recurment tension headache in adolescents treated with selfhelp relaxation training and a muscle relaxant drug. Headache 1990;30:665-671.

47. MaGrath PJ, Humphreys P, Goodman JT, Keene D, Firestone P, Jacob P, Cunningham SJ. Relaxation prophylaxis for childhood migraine: a randomized placebo-controlled trial. Developmental Medicine and Child Neurology 1988;30:626-631.

48. Olness K, MacDonald JT, Uden DL. Comparison of self-hypnosis and propranolol in the treatment of juvenile classic migraine. Pediatrics 1987:79:593-597.

49. Passchier $J$, van den Bree MB, Emmen HH, Osterhaus SO, Orlebeke JF, Verhage $F$. Relaxation training in school classes does not reduce headache complaints. Headache 1990; 30:660-664.

50. Richter IL, McGRath PJ, Humphreys PJ, Goodman JT, Firestone P, Keene D. Cognitive and relaxation treatment of paediatric migraine. Pain 1986:25:195-203. 


\section{Chapter 2}

\section{Recurrent headache, coping and quality of life in children: a review}

Bandell-Hoekstra I, Huijer Abu-Saad H, Passchier J, Knipschild P. Recurrent headache, coping and Quality of Life in children: a review. Headache 2000:40:357-370. 


\section{ABSTRACT}

Purpose of this article is to clarify the concepts of pain coping and Quality of Life (QoL) and to present a literature review on the strategies that children with recurrent headaches use to cope with their pain, the impact of recurrent headaches on children's QoL, and the influence of personal and situational variables on headache, coping and QoL in children. The literature search encompassed published articles that were found by means of a CD-ROM search of Medline (1966-December 1998) and Psychlit (1974- December 1998) and the snowball method.

In pediatric headache research, only 3 studies have been found in which children report the use of variaus coping strategies, and only 2 studies involving QoL. Demographic factors and psychological variables such as depression, anger and anxiety influence headache prevalence. The impact of headache-related variables such as headache type, severity, perceived cause and prior experience on QoL has only been studied in adults. More research on coping and QoL is needed in pediatric headache. The conceptual model that is presented in this article may serve as a guide. 


\section{INTRODUCTION}

Primary headaches occur frequently in children. Without distinguishing between different headache types, recurrent headaches of once a week or more often are reported by more than $15 \%$ of schoolchildren. ${ }^{1-4}$

Headache affects the individual, the family and society. ${ }^{5}$ The impact of headache in children has been studied in terms of school absenteeism and medical consumption. ${ }^{6-9}$ Recently, Quality of Life (QoL) has been recognized as a major outcome measure of the impact of headache and its treatment. ${ }^{10,11}$ The impact of headache on a child's QoL is determined by the ability to cope with headache. ${ }^{12,13}$ In terms of the stress-coping paradigm ${ }^{14}$, headache can be viewed as a stressor which requires coping. ${ }^{15}$ The underlying assumption of this approach of coping and QoL is that headache has an impact on QoL, which is influenced by coping strategies.

Another approach to coping and QoL assumes that dissatisfaction with important aspects of life becomes manifest in headaches through the influence of stress. ${ }^{16}$ The theary of stress as a headache trigger seems to be physiologically plausible for migraine and tension-type headache. ${ }^{17,18}$ Indeed, children perceive stress as an important trigger of their headaches. 1,2,19,20 The underlying assumption of this approach of coping and QoL is that a low or diminished QoL leads to stress. Coping strategies to manage the stress may be insufficient so that the stress may trigger headache.

The two approaches of the relationship between coping and QoL in pediatric headache can be summarized in a conceptual model (figure 2.1).

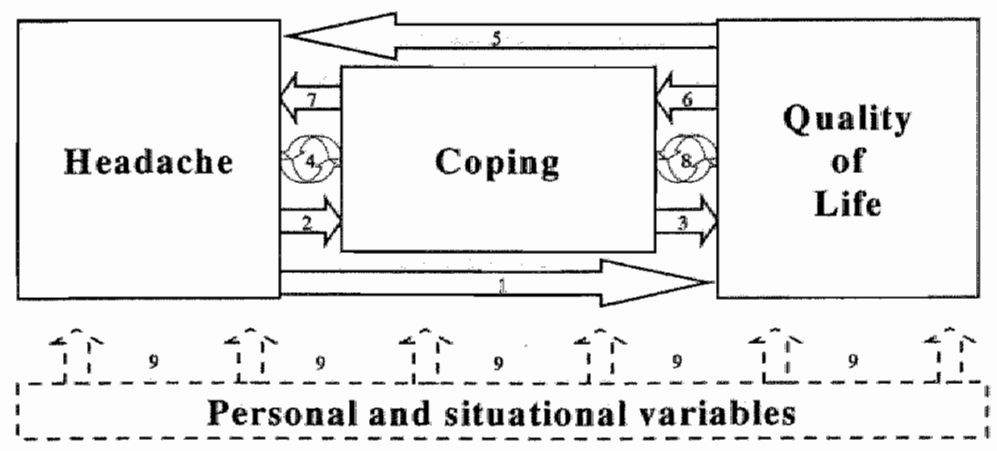

Figure 2.1 Conceptual model linking primary headache, coping and Quality of Life in children 
The model assumes that headache has an impact on the perceived QoL (arrow 1). Headache needs to be dealt with by using coping strategies (arrow 2). The coping strategies applied influence the QoL (arrow 3). Utilizing strategies to cope with headache, regardless of the effect on QoL, influences the experience of headache as in a feedback-loop (arrows 4). A diminished or low QoL can be perceived as a stressor, which triggers, maintains or exacerbates headache (arrow 5). Coping strategies are required to deal with the stress (arrow 6) and influence the perceived headache (arrow 7). The strategies to cope with the stress may impact on the QoL as in a feedback-loop (arrows 8).

Headache, coping and QoL interplay in a setting of personal and situational variables (arrows 9). Demographic and personal characteristics, headache characteristics, and features of the physical and social environment 21 contribute to the link between headache, coping and QoL.

The aim of this article is to clarify the concepts of coping and QoL and to present the state of the art on coping and QoL in pediatric headache research. Variables influencing headache, coping and QoL in children will be reviewed.

The literature search encompassed published articles that were found by means of a CD-ROM search of Medline (1966- December 1998) and Psychlit (1974December 1998), using the search terms "headache, children, coping". "headache, children, Quality of Life", "headache, children and psychology". In addition, the snowball method has been used.

\section{COPING}

Coping can be defined as intentional cognitive and behavioral efforts to manage specific external or internal demands (and conflicts between them) that are appraised as stress because they are taxing or exceeding the resources of the person. ${ }^{22}$ Coping is a process and includes a coping goal, the coping response itself (i.e. the strategy), and coping outcome. ${ }^{15,22}$ Instead of coping strategy. coping skill has been suggested in order to underscore the view of coping as an ability that can be taught and used flexibly as the situation demands. ${ }^{21}$ On the contrary, coping style suggests a generalized strategy or habitual preference for approaching problems, irrespective of their source or nature. ${ }^{23}$

Classification of coping strategies into problem-focused versus emotion-focused coping distinguishes strategies into those directed at the distressing situation and those aimed at regulating emotions that are evoked by the event. ${ }^{14}$ 
Another classification distinguishes approach versus avoidance styles of coping, incorporating information seeking versus information avoiding, attention versus distraction, and active versus passive strategies. ${ }^{15}$

An important theoretical component of the coping process is cognitive appraisal. Cognitive appraisal is the conscious or unconscious process of categorizing an encounter, and its various facets, with respect to its significance for well-being. 14 Coping is mediated by primary and secondary cognitive appraisal. Primary appraisal concerns the question "Am I in trouble or not?", and secondary appraisal "What, if anything, can I do and what are the consequences?".

When pain is the stressor that requires coping strategies, pain beliefs contribute to the process of appraisal and coping. They concern, for example, thoughts on the general locus of control, the controllability of pain specifically, attributions about one's own ability to use specific pain responses, cognitive errors, and outcome expectations. ${ }^{24}$

The assessment of pain coping strategies in children and adolescents can be done by means of a structured interview 25,26 , by showing videotaped vignettes of a child in pain and asking the child the likelihood of employing several proposed strategies ${ }^{27}$, or by using self-administered questionnaires. ${ }^{28-31}$ Structured interviews have shown that 6-12-year-old children with migraine use affective coping in the form of seeking social support to manage the emotional responses to their headaches. In addition, they use problem-focused coping and cognitive coping, in the form of thought-stopping, spiritual coping, and mental distraction. ${ }^{26}$ Schoolchildren mentioned rest and relaxation as coping strategies most often, followed by cooling, analgesics, and distraction. Doing nothing to deal with their headaches was reported by $18 \% .{ }^{25}$

The KidCope questionnaire ${ }^{30}$ has demonstrated that preferred pain coping strategies of children suffering from recurrent headaches are (in descending order): taking medicine or lying down, distraction, relaxation, seeking family support, wishful thinking, becoming helpless, problem-solving, maintaining a future orientation, and remaining positive. Differences across headache types are found in that children with chronic daily headache are more likely to blame others, use problem-solving or attempt to forget the pain, when compared to migraine or mixed headache sufferers.

The effectiveness of coping strategies has been expressed in terms of traitanxiety, missed days of school, expression of more negative behavior, more pain expression, more off-task behavior, and pain-related interference with regular activities of daily living. ${ }^{32}$ In addition, high behavioral problems together with low social competency scores and school absenteeism define "non-copers" among 
pediatric migraine sufferers. ${ }^{26}$ Non-copers were found to have a narrower range of coping repertoire than copers. They depended more on social support from specific family members only, instead of from a wider, interpersonal network. They reported less use of preventive and anticipatory coping, and emphasized cognitive and problem-focused coping less.

In an experimental setting, non-coping adolescents with chronic benign intractable pain showed more negative behavior (i.e., verbally indicating anger, refusal or discouragement) when they had to perform a task. They tended to complain of pain more, gave up more easily and displayed non-compliance. ${ }^{33}$ In general , avoidance coping appears to be associated with more positive adaptation in the short-run, while approach coping will be associated with more positive outcomes in the long-run. ${ }^{34}$

The assessment of successful coping requires an interactional approach of person and situation. It involves at least a match between the stressor (i.e., headache) and coping and, specifically in children, it needs a match between self-initiated coping and external-initiated (e.g., parent) coping. ${ }^{15}$ Assessment of a match between self-initiated coping and external-initiated coping requires the assessment of the perceived, reported or observed influence of parents (or others), on the pain coping of the child.

In pediatric headache, the focus on coping has been primarily through cognitivebehavioral training programs. Teaching coping strategies to deal with stress and pain appears to be effective in reducing headache frequency. ${ }^{35,36}$

\section{QUALITY OF LIFE}

QoL is a concept that encompasses a broad range of physical and psychological characteristics and limitations that describe an individual's ability to function, and the satisfaction derived from doing so. ${ }^{37}$ Health-related QoL is an expression of individuals' perceptions of their position in life in the context of the culture and value systems in which they live and in relation to their goals, expectations, standards and concerns. It is affected by their physical health, psychological state, level of independence, social relationships, and relationships to salient features of the environment. ${ }^{38}$ Health-related QoL is closely related to the World Health Organization (WHO) definition of health ${ }^{39}$, and includes concepts such as disease, physical well-being and role-limitations, social wellbeing, general health perceptions and satisfaction. ${ }^{40}$ Other definitions include psychological functioning as well, encompassing stress and depression among other things. ${ }^{41}$ Thus, health-related QoL is not only a multidimensional concept, 
incorporating social, psychological and physical health. It is also a double-sided concept because it incorporates positive as well as negative aspects of wellbeing and life. In addition, it is a dynamic concept that changes when health status changes. But most importantly, it is a personal subjective concept that should be self-assessed. ${ }^{42,43}$

In the light of this description, single indicators such as mortality and morbidity can no longer be viewed as representing health-related QoL. However, whether health-related QoL should be measured by means of profiles that produce scores on several dimensions of QoL, or by means of composite indexes that aggregate several measures into one overall index, is still under debate. ${ }^{40,44}$

Basically, health-related QoL can be assessed by means of disease-specific or generic measures. ${ }^{11}$ Generic questionnaires allow the possibility to compare QoL across diseases, and clinical and research settings. The advantage of diseasespecific QoL questionnaires is the emphasis on disease-specific concerns and needs, ensuring sensitivity to clinically significant changes in health status and disease severity $11,40,43,44$ and thereby improving reliability, validity and acceptability. ${ }^{44}$ Alternatively, a standardized, generic instrument to which disease-specific supplements can be added has been proposed. ${ }^{40}$

The assessment of headache-related QoL in children and adolescents has only recently started. In adolescents, an ad hoc questionnaire on QoL ${ }^{16}$ led to the development of the Quality of Life Headache in Youth (QLH-Y) questionnaire. 41,45 , addendum 1

In male adolescents, low correlations have been reported between headache frequency, intensity and duration, and dimensions of QoL. Headache frequency correlated negatively with satisfaction with health. Headache duration correlated negatively with satisfaction with autonomy, and intensity correlated negatively with satisfaction with health, satisfaction with school, satisfaction with home situation, general satisfaction, and satisfaction with autonomy. ${ }^{16}$ In adolescents with chronic headache and migraine, 4-week diary measurements of headache intensity and frequency have been compared with simultaneously recorded data on the QLH-Y questionnaire. The subscales "headache impact on daily activities", "harmony", "fatigue", "cheerful mood/good humor", "satisfaction with life in general" and "satisfaction with health" proved to be sensitive to changes in actual headache presence in the expected direction. ${ }^{45}$

sudendum I Recently, a migraine-specific disability tool (PedMIDAS) was adapted for children and adolescents. (Hershey AD, Powers SW, Vockell A-LB, LeCates S, Kabbouche MA, Maynard MK. PedMIDAS -

Development of a questionnaire to assess disability of migraines im children. Neurology 2001;57:2034-2039. 
Health-related QoL in headache research is important for the assessment of aspects of the burden of headache on individual sufferers, as an outcome measure in clinical trials or clinical practice, for the identification of optimal treatments and (re)allacation of resources, and for the comparison of the burdens as well as the therapeutic opportunities of various disease categories. ${ }^{10}$ No studies have been found that address these functions of QoL in children and adolescents.

In adults, however, migraine sufferers reported substantially diminished functioning and well-being, compared to the general population. ${ }^{46,47}$ Differences between headache sufferers and general population seem to be small though. 48 Largest differences have been found in measures of bodily pain, role disability due to physical health, and sacial functioning ${ }^{46}$ in addition to vitality ${ }^{48}$ and role disability due to emotional problems ${ }^{47}$. Even in between attacks, QoL seems to be impaired when compared to headache-free controls. ${ }^{49}$

Compared to patients with other chronic illnesses, adult patients with chronic headache show significantly worse physical, social, and role functioning, and worse mental health. Health-related QoL of chronic headache sufferers seems to be comparable to the QoL after recent myocardial infarction or congestive heart failure, but lower than QoL related to arthritis, diabetes, depression and back problems. ${ }^{50}$, addendum 2

QoL assessment has been an outcome measure of pharmacological therapeutic trials in adult headache sufferers. ${ }^{11,51-54}$ Only one recently published study using QoL as an outcome measure in a non-pharmacological trial was found. ${ }^{55}$

\section{VARIABLES INFLUENCING HEADACHE, PAIN COPING AND QOL}

Headache, pain coping and QoL are influenced by pre-existing variables that reflect characteristics of the child and of the situation in which the child has to deal with the headache.

Person-specific variables studied in regard to headache, pain coping and QoL encompass demographic variables such as age, gender and socioeconomic status, and psychological state and trait variables. Situation-specific variables include characteristics of the headache present, contrallability of the headache as a

\footnotetext{
addeadum 2 When compared to abdomimal pain and limb pain, the relatively poorest QoL is found in adolescents with headache or back pain. Twice as many adolescents with headaches report school absence, in oontrast to adolescents with pain at the limbs, abdomen or back. (Hunfeld JAM, Passchier J, Perquin CW, HazebroekKampschreur AAJM, van Suijlekom-Smit LWA, wan der Wouden JC. Quality of life in adolescents with chronic pain in the head or at other location. Cephalalgia 2001; 21:201-206).
} 
consequence of perceived triggers and expectations based on prior pain experiences, and the context in which the headache starts.

\section{Person-specific variables: Demographic variables}

Studies on the frequency of headache in children have shown an interaction between age and gender. Until the age of 10 to 12 , the prevalence of headache is as common in boys as in girls. During and after puberty headache is more prevalent in girls. ${ }^{2,56-60}$

Not only the frequency of headache changes during growing up, pain coping strategies develop also.

The number of spontaneous pain coping strategies increases with age, specifically in respect to cognitive strategies such as positive self-statements and attention diversion. ${ }^{32,61}$

Developmental stage-related changes in children's definitions and beliefs about pain, reported among both healthy ${ }^{62}$ and chronically sick ${ }^{63.64}$ children, may influence the development of the pain coping process, just as developmental changes in peer friendships and parental support. ${ }^{65,66}$

With regard to gender and pain coping strategies in general, 9-12-year-old girls $(58 \%)$ rather than boys ( $18 \%$ ) appear to be able to spontaneously present two or more strategies to cope with pain. Moreover, girls report a larger variety of strategies. ${ }^{61}$ Opposite results have been found in children and adolescents with chronic headaches in the United States. Male patients reported the use of a higher number of coping skills. Surprisingly, a 3-way interaction was found between sex, race and headache type. Irrespective of the headache type, minority males displayed the highest total number of coping strategies, while Caucasian boys reported a decrease of number of strategies from migraine, to mixed and chronic daily headache, respectively. In girls, total number of coping strategies was equal for migraine, higher for Caucasians in mixed headaches and higher for minorities in chronic daily headache. ${ }^{30}$

Gender differences in coping may reflect differential socialization, or mare inherent differences in the developmental sequence or speed of each gender. ${ }^{23}$ No studies were found in the field of pediatric headache, reporting age and gender differences with regard to QaL.

\section{Person-specific variables: Psychological functioning}

Several studies have suggested a relationship between chronic (headache) pain and personality and psychological characteristics of the child.

Children suffering from recurrent headaches, including migraine, seem to score higher on depression than control children ${ }^{3,67,68}$, with younger children twice and 
older children three times as high. ${ }^{69}$ Forty-one percent of 9-12 year old girls diagnased with depression reported headaches compared with $9 \%$ of girls without depression, or $9 \%$ of boys in this age-group. ${ }^{70}$ In adolescents, headache is the somatic complaint that shows the highest correlation $(r=.48)$ with depression. ${ }^{71}$ Headache is twice as cammon in depressed as in non-depressed adolescents. ${ }^{72}$ Although not all pediatric studies confirm these findings ${ }^{73.74}$, a co-morbidity of migraine as well as tension-type headache and depression has been reported in adults. ${ }^{75,76}$

The relationship between depression and headache may be bi-directional. ${ }^{75}$ Either way, somatic focus seems to be a mediator of the relationship. According to the neurobiological model ${ }^{77}$, depression is accompanied by a higher degree of somatic focus, through which pain facilitation neurons will be activated, causing a stimulus to be perceived as more intense, i.e. the threshold for perception of somatic pain decreases. ${ }^{58,78}$ The psycho-dynamic model hypothesizes that pain may lead to higher somatic focus, and therefore increase depression. 77 Indeed, a positive association between headache, depression and somatic concern has been reported in children with recurrent headache. ${ }^{69,73}$ Moreover, pediatric headache sufferers show more somatic symptoms and pain complaints, when compared to headache-free controls. ${ }^{82}$ Although evidence remains inadequate to substantiate the view that depression precedes and generates pain in the majority of chronic pain sufferers ${ }^{79-81}$, a recent longitudinal study suggests an increased risk for early adult headache when suffering from depression as an adolescent. ${ }^{72}$, addendum 3

Depression does not only influence the sensory aspects of pain, as can be explained by the Gate Control Theory ${ }^{83}$, but in addition moderates the affective and evaluative dimensions of pain. The influence of depression on pain experience may be mediated by catastrophizing thoughts. In a path-analysis study of adults, catastrophizing was found to be slightly to moderately related to the sensory, evaluative and affective dimensions of pain. ${ }^{84}$

Among adults with chronic pain, depression has been reported to be associated with higher levels of self-reported pain and pain behavior, lower levels of physical and psychosocial functioning and poor response to treatment. ${ }^{85-88}$ Chronic pain is found to be related to more current depression and less current life satisfaction. ${ }^{89}$ Thus, depression might be a mediator of the relationship
between (migraine) headache and QOL.

\footnotetext{
headache in 1996, in girls. (Rhee $H$ Prewallencewed that depression and low self-esteem in 1995 preceeded $2000 ; 40: 528-538$ ).
} 
Suppressed anger appears to be related to depression. ${ }^{90}$ The direction of the association between pain and anger remains unclear though. Internalized anger may be depressed as pain, or inhibited anger may exacerbate chronic pain. ${ }^{91}$ Anger and related concepts such as frustration, hostility, and aggression have not been studied in children with headaches. However, temperament may influence a child's responsiveness to stress and the style of coping, by tempering psychological and biological preparedness to respond to stress. ${ }^{92}$ Features of temperament, viewed as the emotional side of a child's personality, seem to be distinct in clinically referred children with migraine, when compared to a control group of schoolchildren. The migraine child shows more hyperactivity, less adaptability, less persistence, higher distractibility and low threshold. ${ }^{93}$

Results on the relationship between headache and anxiety are inconclusive. State-trait anxiety scores in children with migraine or recurrent headaches were found within a normal range and comparable to those of headache-free controls. ${ }^{67,73,74}$ However, migraineous children with higher self-rated anxiety experienced more frequent and severe headaches. ${ }^{67}$ Other studies found elevated trait-anxiety scores in children with recurrent headaches, when compared to controls. $3,68,69,94$ In children with recurrent headaches, the level of anxiety seems to increase from childhood through adalescence. ${ }^{95}$ The influence of anxiety on pain experience may be mediated by attention that is paid to the painful situation because of anxiety. ${ }^{96}$

Fear of failure appears to be associated with headache frequency, intensity, and attributed trouble and consequences, in children 10-17 years of age. ${ }^{2}$ In adults, fear of failure is more often found in headache patients than headache-free controls. ${ }^{97}$ In the latter study, achievement motivation was found to be more prevalent in clinical headache patients. In addition, higher achievement motivation has been reported in adolescents who suffered from tension-type headaches, with an interaction between intelligence, desire for success, and achievement. ${ }^{98}$ Adolescents with recurrent headaches seemingly spend more time on their homework than headache-free controls. ${ }^{3}$ In a non-clinical population, however, the relationship between achievement motivation and headache measures in adolescents could not be confirmed. ${ }^{2}$

Migraine sufferers might be more vulnerable to psychopathology and poor adjustment to medical condition. In young adults, after adjustment for gender and history of major depression and anxiety disorders, migraine was found to be associated with neuroticism, but not with extraversion or psychoticism. ${ }^{99}$ As compared to headache-free controls, more nervous problems were found in adolescents with recurrent headaches. ${ }^{3}$ With regard to QoL, neuroticism seems to be strangly correlated with negative affects, while extraversion seems 
related to positive affects. Self-esteem and general well-being appear to be positively associated. ${ }^{100}$

The Personality Inventory for Children (PIC) may be useful in distinguishing migraineous children from control children with regard to personality. ${ }^{68,101}$ An intellectual inhibition, inhibition of psychomotor activity and aggressiveness, inhibition of affect, ineffective use of defense against anxiety, prevalence of phobic features and massive use of repression was found in children with migraine when compared to matched controls, using the Rorschach test of personality and temperament. ${ }^{102}$

One might conclude that a wealth of studies suggests a relationship between headache and personality traits and psychological functioning. However, the addition of a "chronic pain other than headache" control group to studies might show that personality and behavioral features, thought to be specifically characteristic of migraine or other headache types, may rather be the result of having a chronic pain disorder ${ }^{73}$. In addition, a prospective, langitudinal cohort study in young adults suggests that personality features associated with migraine represent effects of a long-lasting migraine disorder, rather than vulnerability factors for migraine. ${ }^{103}$ More research is thus warranted.

\section{Situation-specific variables: Headache-related}

Cross-situational variability can be headache-related or due to characteristics of the environment. Headache-related variability involves at least the type and stage of the headache and its controllability. Diagnostic criteria as suggested by the International Headache Society ${ }^{104}$ distinguish headache types by means of headache cause, i.e., primary versus secondary headache, headache chronicity, pain characteristics including location, intensity and quality, and accompanying symptoms (e.g., nausea, photophobia, phonophobia). The stage of the headache refers to the sequence of anticipation, encounter, and recovery. ${ }^{15}$

Adult tension-type headache sufferers only vary from migraine sufferers with regard to coping responses to headache by less avoidance or reduction of lights, less reduction of social contacts, less use of sleep and more trying to go on despite pain. However, pain intensity, and not diagnostic category or headache frequency, was significantly related to coping behavior. 105,106

QoL measures show, at least in adults, that tension-type headache is associated with lower mental health and less social functioning than migraine. ${ }^{107}$ This is not confirmed in another study, however, which found the QoL of tension-type headache sufferers as well as of other headache sufferers comparable to or higher than that of migraine sufferers. 47 During an attack, patients with 
episodic headaches are found to be more disabled than chronic headache sufferers. The physical symptoms force them to lie down, thus interfering more deeply with social life. Chronic headache sufferers, however, report more emotional disturbances. In between attacks, though, the QoL of chronic daily headache sufferers remains more compromised. It involves avoidance of social life and smoking, worrying about whether a headache would start or could worsen, fearing that others would not understand it, interference with social life, and feeling fed up, not in control, frustrated, irritable, worried and being robbed of one's own time. ${ }^{108}$

When confronted with headache, secondary appraisal involves children's perception of control. Perception of control may be determined by prior experiences of headache, pain and coping strategies, perceived self-efficacy and perceived headache triggers. Untill the age of 12 children display a rather unidimensional definition of pain, in which they attribute their pain mainly to clearly related and immediate causes. ${ }^{109}$ However, children who suffer from severe headaches attribute their pain mainly to tension as a result of a timepressured daily routing, a cause that apparently had been suggested by their pediatrician. ${ }^{109}$ Indeed, parental and doctor's influences on children's perceived causes of headache must be reckoned with. ${ }^{2}$ Stress is the main headache trigger identified by children. ${ }^{2,19,20,67}$ Younger children seem less likely to acknowledge that something has triggered their headache on the day of the episode or on the day before. An especially hard day at work, school, or home, an unpleasant emotional situation, worrying a lot, unexpected excitement or pressure, tension, and happy or sad emotions were identified as stress-related headache triggers by most children, when asked in a closed-answer format. ${ }^{20}$ In a 10-17-year-old population of school children, open-ended questions on perceived headache triggers reveal, in addition to stress as the major headache trigger, lack of sleep, visual effort, heat, head injury, physical effort, suffocating atmosphere, infectious disease, noise, and intense light. ${ }^{2}$ Migraine patients 6-16 years of age report, again in an open-ended answer format, stress, bright light, overtiredness, exercise, missing meals, and certain foods to be possible headache triggers. One quarter of the children have no idea of any trigger. ${ }^{67}$ of elementary and secondary schoolchildren $62 \%$ and $52 \%$ respectively do not know what causes their headache. ${ }^{2}$ Unfortunately, no studies have been found on the relationship between perceived trigger and subsequent pain coping behavior.

Prior experiences influence pain beliefs and thus appraisal. More experience does not necessarily imply more control or the opportunity to develop adaptive coping behaviors. ${ }^{110}$ On the contrary, negative experiences may sensitize a child 
to more intense feelings of pain in adolescence, compared to childhood. In a study of adolescents with juvenile rheumatoid arthritis, the pain appeared to remind the older patients of the implications of the disease, such as the possibility that the disease would affect their life and might become worse. These beliefs may have increased the severity of the pain that the patients were experiencing. ${ }^{64}$

Contributing to the development of pain beliefs is perceived self-efficacy. Efficacy expectations influence the extent to which a coping behavior will be attempted, how much persistence will be shown, and the eventual outcome. ${ }^{110}$

In addition, self-efficacy moderates the relationship between stress and headache. In adults who perceive a low capacity to exercise self-control over responses to stressful events, the correlation between the frequency of stressful events and headaches is high. Increasing self-efficacy decreases the relationship between stressful events and headaches. ${ }^{111}$

\section{Situation-specific variables: Environment}

The way children experience their headache is influenced by many characteristics of the environment. The role of the family, parents or caregivers in particular, is mostly prominent. Families can contribute to the pain etiology, maintain the pain problem and deal with the impact of the pain on the family. ${ }^{112}$ A positive family history of headache has been frequently reported, specifically for migraine $3,58,67,113,114$, but also for tension-type headache ${ }^{115}$. Along with the heredity of migraine ${ }^{116}$, madeling may have an effect on the learning of cognitive processes that affect pain, as well as on the development of pain behaviors. $117-119$ Children of chronic headache sufferers experience more headaches per month, appear to be more concerned with their body image, and report less energy than their control counterparts. ${ }^{120}$

Pain behaviors may be seen as operant behaviors that increase in frequency when the patient receives desirable consequences or is able to avoid undesirable activities contingent upon displaying pain behaviors, i.e. "secondary gain". 121 Caregivers provide discriminative cues and selective reinforcement for behavioral expressions of pain. Parental attention contingent on pain, the avoidance of non-preferred activities ${ }^{122}$, and parental modeling ${ }^{123}$ may affect the child's pain. Consequently, training parents to alter attending behaviors that reinforce pain behaviors into supporting their children's well behavior or active coping may be useful. 27,124 Differences in mother-child interactions of adolescents who adequately or inadequately cope with chronic intractable benign pain, show that mothers of non-copers more frequently discourage coping
behavior 33 
Family problems can also have a negative effect on the headache in children. ${ }^{19}$ Adolescents with recurrent headaches are more likely to have parents who are divorced than headache-free controls. ${ }^{3}$ addendum 4

The relationship between culture and pain has been reported since the 1950 s. $^{125}$ Studies on cultural differences in the evaluative dimension of pain in pediatric headache sufferers have not been found. However, prevalence figures from population-based studies of headache types in children and adolescents, mainly conducted in the Western world, appear to be internationally comparable. ${ }^{1-}$ $4,7,25,53,126-136$

\section{CONCLUSION AND DISCUSSION}

The associations between headache, pain coping and QoL are still hypothetical. Clearly, children use various coping strategies to deal with their headaches. Only two studies were found however, involving clinically sampled children 26,30 , in addition to one population-based study using interviews ${ }^{25}$. The results of these studies show that almost all children with recurrent headaches report taking medication/lying down, followed by distraction, relaxation, seeking family support, wishful thinking, becoming helpless, problem-solving, maintaining a future orientation and remaining positive. ${ }^{30}$ In addition, forms of thoughtstopping, methods of mental distraction, humor, sleep, and preventive and anticipatory strategies, have been mentioned ${ }^{26}$, as well as doing nothing 25 . Children seeking help for their headache problem may use coping strategies that are different from children who do not. In particular population-based studies are required to gain more insight in children's pain coping strategies, taking into account help-seeking behavior and headache severity.

Besides, assessment of coping strategies in pediatric headache requires a validated questionnaire that is specifically designed for measuring pain coping. such as the PCQ. ${ }^{31}$ Content validity of the PCQ for headache still needs to be established.

In addition, many questions regarding coping and headache remain unanswered. Cognitive appraisal in the form of pain beliefs that influence the choice of pain coping strategies has not been studied in pediatric headache yet. Personspecific characteristics such as age and gender are obviously related to

addendum 4 This phenomenon was found only in adolescents with tension-type headache, not in migraineurs. (Karwautz A, Wöber C, Lang T, Bôck A, Wagner-Ennsgraber, Vesely C, Kienbacher C, Wöber-Bingöl C.. Psychosocial factors in children and adolescents with migraine and tension-type headache: a controlled study and review of the literature. Cephalalgia 1999;19:32-43. 
headache prevalence, but their relationship to coping strategies is still unclear. Certain psychological variables have been found to influence headache occurrence: depression, somatic focus, catastrophizing, anger, anxiety, fear of failure, achievement motivation, and neuroticism. Again, no studies have been found that focus on the impact of these variables on the use of coping strategies.

Although situation-specific characteristics such as headache intensity and chronicity have been shown to impact on pain coping in adults ${ }^{103,104}$, studies in children are lacking. Headache characteristics, accompanying symptoms and perceived headache cause should be taken into account when studying coping strategies in children. External influences on children's pain coping strategies, coming from parents and peers, present an area open for investigation too.

The impact of recurrent headaches on children's QoL requires similar reflections and directions for future research.

Passchier and van Knippenberg ${ }^{137}$ have reviewed several studies that focused on aspects of QoL, such as somatic complaints and general illness as a part of physical well-being: anxiety, depression, emotional inhibition and stress as a part of psychological well-being; school absence, school achievement, intelligence and social dysfunction as a part of daily functioning; and satisfaction with health or happiness as a global evaluation. However, only two studies have investigated QoL as a multidimensional phenomenon in pediatric headache, both of which focus on the design of the QLH-Y questionnaire for children 12 years of age and older. ${ }^{41}$ Adaptation of the questionnaire for use in younger children needs further attention.

In particular for the evaluation of therapeutic strategies, a larger body of studies that include QoL measures is needed. Such studies could also shed light on the direction of the relationship between headache and QoL. Studies in adults ${ }^{46-50}$ and adolescents ${ }^{45}$ have been cross-sectional untill now. Although QoL in adolescents appears to be sensitive to changes in actual headache presence 45 , the question whether headache impacts on QoL, whether poor QoL triggers the onset of headache, or whether the relationship between headache and QoL is of a reciprocal nature, should be investigated. Studies in adults show a diminished QoL in headache sufferers when compared to the general population. In male adolescents, the headache frequency, duration and intensity have been found to correlate negatively with satisfaction with the different aspects of QoL. ${ }^{16}$ Variables influencing headache, coping, and QoL form a complex entity, which involve person-specific and situation-specific characteristics and the relationship between both. More research is needed to unravel these relationships. 
Focus in pediatric headache studies should not only be on the relationship between headache and pain coping and headache and QoL, but should also include the relationship between pain coping and QoL. In addition, the nature of the relationship between headache, coping and QoL needs to be addressed. The question remains whether coping is a moderator or a mediator ${ }^{138}$ of the relationship between headache and QoL. A theoretical discussion as well as a statistical underpinning of the moderator-mediator distinction with regard to coping in pediatric headache is needed. In this respect, the conceptual model that has been presented in this article may serve as a guide.

\section{REFERENCES}

1. Egermark-Eriksson I. Prevalence of headache in Swedish schoolchildren, Acta Paediatr Scand 1982;71:135-140.

2. Passchier J, Orlebeke JF. Headaches and stress in schoolchildren: an epidemiological study. Cephalalgia 1985:5:167-176.

3. Larsson B. The role of psychosocial, health behavioral and medical factors in adolescent headache. Dev Med Child Neurol 1988;30:616-625.

4. King NJ, Sharpley CF. Headache activity in children and adolescents. I Paediatr Child Health 1990:26:50-54.

5. Stewart WF, Lipton RB. Societal impact of headache. In: Olesen J, Tfelt-Hansen P, Welch KMA, eds. The headaches. New York: Raven Press, 1993:29-34.

6. Collin C, Hockaday JM, Waters WE. Headache and school absence. Arch Dis Child 1985:60:245-247.

7. Linet MS, Stewart WF, Celentano DD, Ziegler D. Sprecher M. An epidemiolagic study of headache among adolescents and young adults. JAMA 1989:261:2211-2216.

8. Stang PE. Osterhaus JT. Impact of migraine in the United Statesi data from the national health interview survey. Headache 1993:33:29-35.

9. Metsähonkala $L$, Sillanpäà $M$, Tuominen $J$. Use of health care services in childhood migraine. Headache 1996;36:423-428.

10. Lipton RB. Health-related Quality of Life in headache research. Editarial. Headache 1995:35:447.

11. Soloman 6D. Evolution of the measurement of Quality of Life in migraine. Neurology 1997;48:510-15.

12. McGrath PJ, Unruh AM. Pain in children and adolescents. Amsterdam: Elsevier Science. Publishers, 1987.

13. Bech P. Quality of Life Measurements in Chronic Disorders. Psychother Psychosom 1993:59:1-10.

14. Lazarus RS, Folkman S. Stress, Appraisal and Coping. New York: Springer, 1984.

15. Rudolph KD, Dennig MD, Weisz JR. Determinants and consequences of children's coping in the medical setting: conceptualization, review, and critique. Psychol Bu/1995;118:328-357. 
16. Van den Bree MBM, Passchier J. Emmen HH. Influence of Quality of Life and stress coping behavior on headaches in adolescent male students: an explorative study. Headache 1990:30:165-168.

17. Andrasik F, Passchier J. Tension-type headache, cluster headache and miscellaneous headaches. Psychological aspects. In: Olesen J, Tfelt-Hansen P. Welch KMA, eds. The headaches: New York: Raven Press Ltd, 1993, p. 489-492.

18. Passchier J, Andrasik F. Migraine. Psychological factors. In: Olesen J, Tfelt-Hansen P, Welch KMA, eds. The headaches. New York: Raven Press Ltd, 1993, p. 233-240.

19. Maratos J, Wilkinson M. Migraine in children: a medical and psychiatric study. Cephalalgia 1982:2:179-187.

20. Leviton A, Slack WV. Masek B, Bana D, Graham JR. A computerized behavioral assessment for children with headaches. Headache 1984;24:182-185.

21. Moos RH, ed. Coping with physical illness. 2. New Perspectives. New York: Plenum Medical Book Company, 1984.

22. Lazarus RS. Emotion and Adaptation. New York: Oxford University Press, 1991.

23. Patterson JM, McCubbin HI. Adolescent coping style and behaviors: conceptualization and measurement. J Ado/1987:10:163-186.

24. Jensen MP, Turner JA, Romano JM, Karoly P. Coping with chronic pain: a critical review of the literature. Pain 1991:47:249-283.

25. Van Frankenberg 5 , Pothmann R, Müller B, Sartory G, Wolff M, Hellmeier W. Prevalence of headache in schoolchildren. In: Gallai V. Guidetti V. Juvenile Headache. Etiopathogenesis, Clinical Diagnosis and Therapy. Amsterdam: Excepta Medica, 1991, p. 113-117.

26. Gilbert MC. Coping with pediatric migraine: differences between copers and non-copers. Child Adal Soc Work J1995;12:275-287.

27. Sanders MR, Shepherd RW, Cleghorn $G$, Woolford $H$. The treatment of recurrent abdominal pain in children; a controlled comparison of cognitive-behavioral family intervention and standard pediatric care. $J$ Cans Clin Psycho/ 1994:62:306-314.

28. Spirito A, Stark LJ. Williams $C$. Development of a brief coping checklist for use with pediatric populations. J Pediatr Psycho/ 1988;13:555-574.

29. Gil KM, Williams DA, Thampson RJ, Kinney TR. Sickle Cell disease in children and adolescents: The relation of child and parent pain coping strategies to adjustment. I Pediatr Psychol 1991:16:643-663.

30. Holden EW, Gladstein J. Trulsen M. Wall B. Chronic daily headache in children and adolescents. Headache 1994:34:508-514.

31. Reid GJ، Gilbert CA, MCGrath PJ. The Pain Coping Questionnaire: preliminary validation. Pain 1998: 76:83-96.

32. Branson SM, Craig KD. Children's spontaneous strategies for coping with pain: a review of the literature. Canad J Behav Scie/Rev Canad Scie Comp 1988;20:402-412.

33. Dunn-Geier BJ, MCGrath PJ, Rourke BP, Latter J, D'Astous JD. Adolescent chronic pain: the ability to cope. Pain 1986;26:23-32.

34. Suls J. Fletcher B. The relative efficacy of avoidant and nonavoidant coping strategies: a meta-analysis. Health Psychol 1985;4:249-288.

35. Richter IL, McGrath PJ. Humphreys PJ, Goodman JT, Firestone P, Keene D. Cognitive and relaxation treatment of paediatric migraine. Pain 1986:25:195-203.

36. Osterhaus SO, Passchier J, van der Helm-Hylkema H, de Jong KT, Orlebeke JF, de Grauw AJ. Dekker PH. Effects of behavioral psychophysiological treatment on schoolchildren with 
migraine in a nonclinical setting: predictors and process variables. I Ped Psychol 1992;18:697-715.

37. Walker SR, Rosser RM, eds. Quality of Life: Assessment and Application Lancaster: MTP Press, 1988.

38. WHOQOL Group. Measuring Quality of Life: The Development of the World Health Organization Quality of Life Instrument (WHOQOL) Geneva: WHO 1993.

39. World Health Organizotion. International Classification of Imparments, Disabilities, and Handicaps. A manual of classification relating to the consequences of disease. Geneva: WHO, 1980.

40. Patrick DL. Erickson P. Assessing health-related quality of life for clinical decision-making. In: Walker SR, Rosser RM, eds. Quality of Life: Assessment and Application Lancaster: MTP Press, 1988, p. 9-49.

41. Langeveld JH, Koot HM, Loonen MC, Hazebroek-Kampschreur AAJM, Passchier J. A quality of life instrument for adolescents with chronic headache. Cephalalgia 1996:16:183-196.

42. Schipper H. Quality of Life: Principles of the clinical paradigm. I Psychosoc Oncol 1990;8:171-185.

43. Bowling A. Measuring disease. Buckingham: Open University Press, 1995.

44. McKenna SP. Quality of Life assessment in the conduct of economic evaluations of medicines. Br J Med Econ 1995;8:33-38.

45. Langeveld JH, Koot HM, Passchier J. Headache intensity and quality of life in adolescents. How are changes in headache intensity in adolescents related to changes in experienced quality of life? Headache 1997,37:37-42.

46. Osterhaus JT. Townsend RJ, Gandek B, Ware JE. Measuring the functional status and wellbeing of patients with migraine headache. Headache 1994;34:337-343.

47. Michel P, Dartigues JF, Lindoulsi A, Henry P. Loss of productivity and Quality of Life in migraine sufferers among French workers: results from the GAZEL cohart. Headache 1997:37:71-78.

48. Essink-Bot M-L, van Royen L, Krabbe P, Bonsel GJ, Rutten FF. The impact of migraine on health status. Headache 1995; 35:200-206.

49. Dahlöf CGH, Dimenas $E$. Migraine patients experience poorer subjective well-being/quality of life even between attacks. Cephalalgia 1995;15:31-36.

50. Solomon GD, Skobieranda FG, Gragg LA. Quality of life and well-being of headache patients: measurement by the medical outcomes study instrument. Headache 1993;33:351-358.

51. Miller DW, Osterhaus JT, Hirsh JD. Quality of life assessment among migraineurs treated with Sumatriptan: preliminary findings. Headache 1993:33:278.

52. Kirchberger I, Bullinger M, Bruggenjurgen B. Quality of life in migraine tmeatment. Results of a longitudinal study using the SF-36 survey. Qual Life Res 1994;3:83.

53. Boureau F, Chazot G, Emile J, Bertin L, d'Allens H. Comparison of subcutaneaus Sumatriptan with usual acute treatments for migraine. Eur Neurol 1995:35:264-269.

54. Solomon GD. Skobieranda FG. Genzen JR. Quality af Life assessment amang migraine patients treated with Sumatriptan. Headache 1995;35:449-454.

55. Mannix KL, Chandurkar RS, Rybicki LA, Tusek DL, Solomon GD. Effect of guided imagery on Quality of Life for patients with chronic tension-type headache. Headache 1999:39:326334.

56. Bille B. Migraine in childhood and its prognosis. Cephalalgia 1981:1:71-75. 
57. Stewart WF. Lipton RB, Cellentano DD, Reed ML. Prevalence of migraine headache in the United States. Relation to age, income, race, and other sociodemographic factors. JAMA 1991:276:64-69.

58. Mortimer MJ, Kay J, Jaron A. Epidemiology of headache and childhood migraine in an urban general practice using Ad Hoc, Vahlquist and IHS criteria. Der Med Child Neural 1992,34:1095-1101.

59. Kristjánsdóttir G. Wahlberg $V$. Sociodemographic differences in the prevalence of selfreported headache in Icelandic school-children. Headache 1993:33:376-380.

60. Sillanpaia $M$. Anttilla $P$. Increasing prevalence of headache in 7-year-old schoolchildren. Headache 1996:36:466-470.

61. Tesler MD, Wegner $C$, Savedra M, Gibbons PT, Ward JA. Coping strategies of children in pain. Iss Compr Ped. Nurs 1981:5:351-359.

62. Steward MS, Steward DS. Children's canceptions of medical procedure. In: Bibace R, Walsh $M E_{1}$ eds. Children's conceptions of health, illness and bodily functions. San Francisco, CA: Jossey-Bay, 1981, p. 67-84.

63. Brewster AB. Chranically ill hospitalized children's concepts of their illiness. Pediatrics 1982:69:355-362.

64. Beales JG, Holt PJL, Keen JH, Mellor VP. Children with juvenile chronic arthritis: their belliefs about their illness and therapy. Annois Rheum Dis 1983:42:481-486.

65. Leiderman PH. Social ecology and childbirth: the newborn nursery as environmentall stressor. In: Garmezy N, Rutter M. Stress, coping d development in children. New York, NY: McGraw-Hill book company, 1983, p. 133-159.

66. Eiser $C$. Coping with chronic childhood diseases: implications for counseling children and adolescents, Couns Psychol Quart 1989:2:323-336.

67. Cooper PJ, Bawden HN, Camfield PR, Camfield CS. Anxiety and life events in childhoad migraine Pediatrics 1987:79:999-1004.

68. Guidetti V. Fornara K, Ottoviano S, Petrill, A, Seri S, Cortesi F. Personality Inventory for Children and childhood migraine. A case-controlled study. Cephalalgia 1987:7:225-230.

69. Andrasik F, Kabela E, Quinn S, Attanasio V, Blanchard EB, Rosenblum EL. Psychological functioning of children who have recurrent migraine. Pain 1988:34:43-52.

70. Egger HL. Angold A, Costello EJ. Headaches and psychopathology in children and adolescents. I Am Acad Child Adolesc Psychiat 1998; 37:951-958.

71. Larsson BS. Somatic complaints and their relationship to depressive symptoms in Swedish adolescents. J Child Psychol Pychiat 1991;32:821-832.

72. Pine DS, Cohen P, Brook J. The association between major depression and headache: results of a longitudinal epidemiologic study in youth. I Child Adol Psychopharmac 1996; 6:153-164.

73. Cunningham SJ. MCGrath PJ, Ferguson HB, Humphreys P, D'Astous J, Latter J, Goodman $J T$, Firestone P. Personality and behavioral characteristics in pediatric migraine. Headache 1987:27:16-20.

74. Kowal A, Pritchard D. Psycholagical characteristics of children who suffer from headache: a research note. $J$ Child Psychol Psychiat 1990;31:637-649.

75. Breslau N, Davis GC, Schultz LR, Peterson EL. Migraine and major depression: a longitudinal study. Headache 1994:34:387-393.

76. Ho K-H, Ong BKC, Lee S-C. Headache and self-assessed depression scores in Singapore University undergraduates. Headache 1997:37:26-30.

77. Fields H. Depression and pain. A neurobiological model. Neuropsychiat Neuropsychol Behav Neuro/1991:4:83-92. 
78. Geisser ME, Gaskin ME, Robinson ME, Greene AF. The relationship of depression and somatic facus to experimental and clinical pain in chronic pain patients. Psychol Health 1993:8:405-415.

79. Romano JA, Turner JA. Chronic pain and depression: does the evidence support a relationship? Psychol Bull 1985;97:18-34.

80. Roy R. Engel's pain-prone disorder patient: 25 years after. Psychother Psychosom 1985:43:126-135.

81. Gupta M. Is chronic pain a variant of depressive illness? Can J Psychiat 1986:31:241-248.

82. Corlsson J, Larssan B, Mark A. Psychosocial functioning in school children with recurrent headaches. Headache 1996:36:77-82.

83. Mellzack R, Wall PD. The challenge of pain. Hamondsworth: Penguin Books, 1982.

84. Geisser ME, Robinson ME, Keefe FJ, Weiner ML. Catastrophizing, depression and the sensory, affective and evaluative aspects of chronic pain. Pain 1994:59:79-83.

85. Blanchard EG, Andrasik $F$, Neff DF, Arena JG, Ahles TA, Jurish SE, Pallmeyer TP, Saunders NL, Teders SJ, Barron KD, Rodichok LD. Biofeedback and relaxation training with three kinds of headache: treatment effects and their prediction. $J$ Consult Clin Psychol 1982:50:562-575.

86. Keefe F. Wilkins R, Cook W, Crisson J, Muhlbaier L. Depression, pain, and pain behavior. $J$ Consult Clin Psychol 1986:54:665-669.

87. Kerns $R$, Haythornthwaite $J$. Depression among chronic pain potients: cognitive-behavioral analysis and effect on rehabilitation outcome. $J$ Consult Clin Psycha/ 1988;56:870-876.

88. Haythornthwaite JA, Sieber WJ, Kerns RD. Depression and the chronic pain experience. Pain 1991:46:177-184.

89. Gamsa $A$. Is emotional disturbance a precipitator or a consequence of chronic pain? Pain 1990:42:183-195.

90. Fernandez $E$, Turk DC. The scope and significance of anger in the experience of chronic pain. Pain 1995;61:165-175.

91. Tschannen TA, Duckro PN, Margolis RB, Tomazic TJ. The relationship of anger, depression, and perceived disability among headache patilients. Heodache 1992;32:501-503.

92. Kagan J. Stress and coping in early development. In: Garmezy N, Rutter $M$, eds. Stress, coping and development in children. New York: McGraw-Hill, 1983, p. 191-216.

93. Ottoviano S, Guidetti V. Innocenzi M, Baldini L, Ottoviano P, Ottoviano C. Temperament and migraine. In: Gallai V. Guidetti V. Juvenile Headache. Etiopathogenesis, Chinical Diagnosis and Therapy. Amsterdam: Excepta Medica, 1991, p. 125-128.

94. Bille B. Migraine in school children. Acta Paed Scand 1962;5:s136.

95. Martin SE, Smith MS. Psychosocial factars in recurrent pediatric headache. Pediatr Annals 1995:24:469-474.

96. Arntz $A$, Dreessen L, Merkelbach $H$. Attention, not anxiety, influences pain, Behow Res Ther 1991:29:41-50.

97. Passchier J, van der Helm-Hylkema H, Orlebeke JF. Personality and headache type: a controlled study. Headache 1984:24:140-146.

98. Rangaswamy K. Tension headaches in adolescents. I Psychol Res 1982:26:70-72.

99. Breslau N, Andreski P. Migraine, persanality, and psychiatric comorbidity. Headache 1995:35:38:2-386.

100. Watten RG, Vassend O, Syversen J-L, Myhrer T. Personallity and Quality of life. SoC Ind Res 1995:35:289-302. 
101. Romoli $M$, Ardovini $C$, Bruni $O$, Diamanti R, Fornara R, Guidetti $V$. Childhood and adolescence headache and migraine: analysis of personality through Personality Inventory for Children. In: Gallai V, Guidetti V. Juvenile Headache. Etiopathogenesis, Clinical Diagmosis and Therapy. Amsterdam: Excepta Medico, 1991, p. 507-509.

102. Guidetti V. Mazzei G, Ottaviano S, Pagliavini M, Paolella $A_{\text {" Seri }} S$. The utilization of the Rorschach test in a case-controlled study. Cephalalgia 1986:61:87-93.

103. Merikangas $K R$, Stevens $D E$, Angst J. Headache and personality: results of a community sample of young adults. I Psychiat Res 1993:27:187-196.

104. Headache Classification Committee of the International Headache Society. Classification and diagnostic criteria for headache disorders, cranial neuralgias and facial pain. Cephalalgia 1988;8(supp| 7):10-73.

105. Appelboum KA, Radnitz CL, Blanchard EB, Prins A. The Pain Behavior Questionnaire (PBQ): a global report of pain behavior in chronic headache. Headache 1988:28:53-58.

106. Scharff L. Turk DC, Marcus DA. Triggers of headache episodes and coping responses of headache diagnastic groups. Headache 1995;35:397-403.

107. Solamon GD. Skobieranda FG, Gragg LA. Does quality of life differ among headache diagnosis? Analysis using the medical autcomes study instrument. Headache 1994: 34: 143147.

108. Cavallini A, Micieli G, Bussone G, Rossi F, Nappi G. Headache and quality of life. Headache 1995:35:29-35.

109. Ross DM. Ross 5A. Assessment of pediatric paini an overview. Issues Compr Pediatr Nurs 1988:11:73-91.

110. Siegel LJ, Smith KE. Children's strategies for coping with pain. Pediatrician 1989:16:110-118,

111. Marlowe N. Self-efficacy moderates the impact of stressful events on headache. Headache 1998:38:662-667.

112. Turk DC, Flor $H$, Rudy TE. Pain and families. I. Etiology, maintenance, and psychosocial impact. Pain 1987:30:3-27.

113. Messinger HB, Spierings ELH, Vincent AJP, Lebbink J. Headache and family history. Cephalolgia 1991:11:13-18.

114. Russell MB, Hilden J, Sirensen SA, Olesen J. Familial accurrence of migraine without aura and migraine with aura. Neurology 1993;43:1369-1373.

115. Turkat ID, Kuczmierczyk AR, Adams HE. An investigation of the aetiology of chronic headache. The role of headache models. Br I Psychiat 1984:145:667-668.

116. Sorensen SA, Araki S. Genetics. In: Olesen J. Tfelt-Hansen P, Welch KMA. The Headaches. New Yark: Raven Press, 1993,21-27.

117. Edwards PW, Zeichner A, Kuczmierczyk $A$, Boczkowski J. Familial pain models: the relationship between family history of pain and current pain experience. Pain 1985;21:379384.

118. Lavigne JV, Schulein MJ, Hahn VS. Psychological aspects of painful medical conditions in children. II. Personality factors, family characteristics and treatment. Pain 1986;27:147169.

119. Ehde $O M$. Holm JE. Metzger DL. The role of family structure, functioning, and pain modeling in headache. Headache 1991;31:35-40.

120. Mikail SF, von Baeyer $C L$. Pain, somatic focus, and emotianal adjustment in children of chronic headache sufferers and controls. SoC Sci Med 1990;31:51-59.

121. Fordyce WE. Behavioral method's for chromic pain and illiness. St. Louis: Mosby, 1976. 
122. Philips $H$. The effects of behavioural treatment on chronic pain. Behav Res Ther" $1987: 25: 273-279$.

123. Whitehead WE, Busch CM, Heller BR, Costa PT. Social learning influences on menstrual symptoms and illness behavior. Health Psychol 1986:5:13-23.

124. Beames $L$. Sanders MR, Bor $W$. The role of parent training in the cognitive behavioral treatment of children's headaches. Behav Psychother 1992;20:167-180.

125. Zborowski M. People in pain. San Francisco: Jossey-Bass, 1969.

126. Dalsgaard-Nielsen $T$. Engberg-Pedersen $H_{*}$ Holm HE. Clinical and statistical investigations of the epidemiolagy of migraine. Dan Med Bul/1970;17:138-148.

127. Oster J. Recurrent abdominal pain, headache and limb pains in children and adolescents. Pediatrics 1972:50:429-436.

128. Sillanpäa $M$. Prevalence of migraine and other headache in Finnish children starting school. Headache 1976:288-290.

129. Deubner DC. An epidemiologic study of migraine and headache in 10-20 year olds. Headache $1977: 17: 173-180$.

130. Sillanpää M. Prevalence of headache in prepuberty. Headache 1983:23:10-14.

131. Sillanpää $M$. Changes in the prevalence of migraine and other headaches during the first seven school years. Headache 1983:23:15-19.

132. Camarda $R M_{*}$ Cammalleri $R_{i}$ Cuccia F, Daniele $O$, LaRocca $D$, Raieli V, Raimondo $D$, Romano $C_{*}$ Rossetti $M$. Santangelo $G$. Epidemiological and clinical study of migraine headaches in a juvenile population. In: Gallai V. Guidetti V. Juvenile Headache. Etiopathogenesis, Clinical Diagnosis and Therapy. Amsterdam: Excepta Medica, 1991, p. 107-108.

133. Alfvén $G$. The covariation of common psychosomatic symptoms among children from socioeconomically differing residential areas. An epidemiological study. Acta Paed 1993:82:484487.

134. Abu-Arafeh I, Russell G. Prevalence of headache and migraine in schoolchildren. $B M J$ 1994;309:765-768.

135. Borge AIH, Nordhagen R, Moe B, Botten G, Bakketeig LS. Prevalence and persistence of stomach ache and headache among children. Follow-up of a cohort of Norwegian children from 4 to 10 years of age. Acta Paed 1994;83:433-437.

136. Barrea LM, Tannhauser M, Rotta NT. An epidemiologic study of headoche among children and adolescents of southern Brazill. Cephalalgia 1996:16:545-549.

137. Passchier J, wan Knippenberg FCE. Relevance and limitations of Quality of Life measurements in juvenile patients with chronic headaches. In Gallai V, Guidetti V. Juvenile Headache. Etiopathogenesis, Clinical Diagnosis and Therapy. Amsterdam: Excepta Medica, 1991, p. 449-455.

138. Baron RM, Kenny DA. The moderator-mediator variable distinction in social psychological research: conceptual, strategic, and statistical cansiderations. I Personal Soc Psychol 1986:51:1173-1182. 
46

Chapter 2 


\section{Chapter 3}

\section{Prevalence and characteristics of headache in Dutch schoolchildren}

Bandell-Hoekstra IENG, Huijer Abu-Saad H, Passchier J, Frederiks CMA, Feron FJM, Knipschild P. Prevalence and characteristics of headache in Dutch schoolchildren. European Journal of Pain 2001:5:145-153. 


\section{ABSTRACT}

Aim of this study is to determine whether there has been an increase in headache prevalence in Dutch children and to compare headache characteristics of children with low, medium and high headache severity.

A sample of 2358 schoolchildren between the age of 10 and 17 years filled out Waters' Headache Questionnaire and the Pediatric Pain Assessment Tool. Results show that $21 \%$ of the boys and $26 \%$ of the girls at elementary school, and $14 \%$ of the boys and $28 \%$ of the girls at high school reported weekly headaches. When compared to figures from a previous study in the Netherlands published in 1985, the prevalence of weekly headaches in 10-17 year olds has been increased with $6 \%$. In boys at elementary school, the prevalence of . headaches with a frequency of a few times a week has been doubled.

Children with low, medium and high headache severity differed with respect to all headache characteristics, i.e. pain quality, accompanying symptoms, warning signals, location, onset, impact of headache, family occurrence, perceived cause, medical consultation, and school absence. 


\section{INTRODUCTION}

Headache is a phenomenon that is characterized by the burden, due to the pain, the recurrence and often life-long experience, and the implication on Quality of Life. In children, the prevalence of recurrent headaches is high. Results of population-based studies using self-report questionnaires in school children suggest percentages of children with monthly headaches ranging between 19$47 \%$, and weekly headaches ranging between $6-29 \%$ (table 3.1 ). The prevalence of more frequent headaches is usually reported to be higher among girls, and in younger versus older boys. ${ }^{1-3}$

Table 3.1 Percentages of children with a frequency of monthly and weekly headaches (monthly figures do not enclose weekly figures) in published population-based studies in school children using self-report questionnaires.

\begin{tabular}{|c|c|c|c|c|c|}
\hline authors & country & age & $n$ & monthly \% & weekly \% \\
\hline Small \& Waters $1974^{\circ}$ & UK & $10-16$ & 690 & $29 \%$ & $11 \%$ \\
\hline Small \& Waters $1974^{\text {b }}$ & UK & $10-16$ & 690 & $21 \%$ & $6 \%$ \\
\hline Egermark-Eriksson $1982^{5}$ & Sweden & $7,11,15$ & 402 & $23 \%$ & $9 \%$ \\
\hline Sillanpaa $1983^{6}$ & Finland & 13 & 3784 & $29 \%$ & $8 \%$ \\
\hline Sillanpaa $1983^{7}$ & Finland & 14 & 2921 & $9 \%$ & $9 \%$ \\
\hline Passchier and Orlebeke $1985^{3}$ & Netherlands & $10-17$ & 2181 & $23 \%$ & $17 \%$ \\
\hline Larsson $1988^{8}$ & Sweden & $16-18$ & 1287 & $20 \%$ & $15 \%$ \\
\hline Larsson $1988^{8}$ & Sweden & $16-18$ & 1564 & $47 \%$ & $14 \%$ \\
\hline King \& Sharpley $1990^{1}$ & Australia & $10-18$ & 900 & $34 \%$ & $29 \%$ \\
\hline Kristjánsdóttir \& Wahlberg $1993^{2}$ & Iceland & $11-12,15-16$ & 2140 & $30 \%$ & $22 \%$ \\
\hline Carlsson $1996^{\circ}$ & Sweden & $7-16$ & 1144 & $26 \%$ & $6 \%$ \\
\hline
\end{tabular}

"mild theadaches only: ${ }^{b}$ severe headaches only

Recently, Sillanpäa and Anttila ${ }^{10}$ have shown a large increase from $14 \%$ to $52 \%$ in the prevalence of present headache in a physical examination of 7 year old children at school entry, comparing findings from similar studies in 1974 and 1992. Monthly headaches were reported by $3 \%$ in 1974 ( $n=1927$ ), as compared to $9 \%(n=1436)$ of 7 year olds in 1992 . Weekly headaches have doubled up to $3 \%$ in this age group.

Internationally, data on increasing headache prevalence are lacking. One study on the prevalence of headaches in school children has previously been conducted in the Netherlands. ${ }^{3}$ After one decade, a similar study is thus warranted to examine whether such an increase is found in other countries too.

Main focus of epidemiological studies is headache frequency. Clinical studies ${ }^{11,12}$ usually combine headache frequency, pain intensity and duration into an overall index of headache severity. Degree of headache severity is taken into account in 
the analysis and presentation of results. In population-based studies on headaches in children, results on headache characteristics have only been described as mean scores of the overall sample. Studies reporting headache characteristics in relation to headache severity are lacking. Knowledge on headache characteristics and headache severity in the population serves problem definition and may contribute to the development and tailoring of therapeutic interventions for children suffering from headaches.

Thus, aim of our study was to investigate whether headache prevalence in Dutch school children has increased since the earlier study by Passchier and Orlebeke ${ }^{3}$, and to describe and compare headache characteristics in school children with different degrees of headache severity.

The following questions will be addressed: 1) what is the prevalence of headache in school children in the Netherlands and are these prevalence figures higher than previous findings? 2) do children with a low, medium or high headache severity differ with regard to headache characteristics?

In order to be able to answer these questions, we conducted a population-based study on headache in school children, with a cross-sectional design.

\section{METHODS}

\section{Subjects}

Subjects are school children in the two highest grades of elementary school and the first four grades of high school, aged between 10 and 17 years. In collaboration with the local Youth Health Department of school physicians and school nurses, all elementary schools and high schools in the City of Maastricht were contacted for participation. Twenty-one of 35 elementary schools agreed to participate, including small as well as large elementary schools situated in all socio-economic areas. Three of six high schools in the City of Maastricht participated. One regional high school near Maastricht was added to the sample. to gain a representative number of children at all four levels of education (lower vocational, middle general, higher general and academic preparatory education) in the Netherlands.

All eligible classes participated, except for one high school including only 3rd grade classes and one high school participating only in case of teacher's absence within a 3-months period. Percentages of participating children within the total population are presented in table 3.2 . 
Approximately $40 \%$ of all children in grade 7 and 8 at elementary schools in the City of Maastricht, have been included in the study. In addition, $20 \%$ of the population of high school pupils in the City and region of Maastricht have participated.

Table 3.2 Number of included participants in every grade at elementary school and high school and percentages of participants out of the total population of that grade in the City (elementary schools) and the City and Region (high schools) of Maastricht!.

\begin{tabular}{rccc}
\hline School type & Grade & \# of participants & \% of population \\
\hline Elementary school & 7 & 472 & $40 \%$ \\
High School & 8 & 468 & $41 \%$ \\
& 1 & 445 & $25 \%$ \\
& 2 & 318 & $18 \%$ \\
& $3^{2}$ & 432 & $25 \%$ \\
\hline
\end{tabular}

'Population data were derived from the database of the Youth Health Care Department, which is linked to the municipal database

${ }^{2}$ Percentages are based on an estimate of the population size, because exact data were nat available.

Children were included only after parental informed consent. Children who were absent at the time of the study, were not included.

\section{Measures}

Measures include demographic variables and headache characteristics and have been assessed by means of a self-report questionnaire. Two pilot-studies have been conducted, one procedural test on the length and difficulty of the questionnaire, and one test-retest (after 4 weeks) on reliability, showing moderate to high reproducibility of all measures.

Demographic variables include sex, age and age-group, with age-group divided into young (elementary school) and older (high school).

Headache characteristics were assessed using an adapted version of Waters Headache Questionnaire (WHQ). 3,13,14 The WHQ was completed with the 0-100 millimeter (mm) Visual Analogue Scale (VAS) on usual and worst pain intensity of the Pediatric Pain Assessment Tool (PPAT) ${ }^{15-17}$ and a 5-point Likert scale on headache burden, ranging from "I am only aware of it when I pay attention to it" to "headache: such that I can't do anything". ${ }^{18}$ 


\section{Procedure}

Within 50-60 minutes, children filled out the questionnaires individually in the classroom setting, after a group instruction on all answering formats by one of the researchers. In elementary school, the first nine items were read aloud and explained by the researcher. Although questionnaires were not filled out anonymously, children were assured that their questionnaires would be handled with discretion.

\section{Data analysis}

All questionnaires were encoded and computerized by independent assistants. Questionnaires of children younger than 10 years of age or aged 18 years and older were excluded, in addition to questionnaires with missing values on headache frequency, intensity or duration. The results in headache frequency have been dichotomized into weekly headaches (summation of one and a few headaches a week) versus less frequent headaches. Differences in headache prevalence within the 1995 data were described. The difference between the 1983 and current data was tested by means of a Chi-square test.

Children who reported not having experienced a headache ever in their lives or in the past year were taken into account in the prevalence figures but were excluded from further analyses. The remaining sample was divided into three groups. The high headache severity group consisted of children who combined a headache frequency of once a week or more often with a usual headache intensity of at least $50 \mathrm{~mm}$ on the VAS, and a headache duration usually lasting more than an hour. The low headache severity group included only children with a headache frequency of once a month or less, a usual headache intensity of less than $50 \mathrm{~mm}$ on the VAS, and a usual headache duration of one hour maximum. The medium headache severity group included all other children. Group differences in headache characteristics were analyzed using descriptive statistics.

\section{RESULTS}

\section{Sample}

Of the 2774 children in the participating classes, $2400(87 \%)$ actually filled out the questionnaires. Non-participation was mainly due to absence due to illness $(6 \%)$, child's refusal $(4 \%)$, and no permission of the parents $(3 \%)$. Of the questionnaires $2358(98 \%)$ were available for analysis: 5 were omitted because the child's age was 18 years or older and 37 were omitted because of missing 
values on headache frequency, intensity or duration items. Mean age of the sample was 13.8 (sd 1.8). Girls were slightly more represented (54\%), especially at high school ( $58 \%)$.

\section{Headache frequency}

Lifetime prevalence in children was found to be $97 \%$. Headache prevalence in the past year was $92 \%$. Prevalence in the past week was $47 \%$, while on the day of assessment $24 \%$ of the children reported having experienced a headache.

Table 3 presents the $1983^{3}$ and current results of headache frequency in total as well as classified into gender by age group according to school-type. Within the 1995 data a substantial difference in weekly headaches was found between boys $(12 \%)$ and girls $(24 \%)$. The difference between children at elementary and high school was particularly found in girls $(20 \%$ versus $27 \%)$.

Table 3.3 Percentages of schoolchildren according to headache frequency, presented by age group of elementary school (10-12 years old) versus high school (12-16 years old) for boys and girls respectively, comparing current results with 1983 data.

\begin{tabular}{|c|c|c|c|c|c|c|c|c|c|c|}
\hline & \multirow{2}{*}{\multicolumn{2}{|c|}{ Tatal }} & \multicolumn{4}{|c|}{ Elementary school } & \multicolumn{4}{|c|}{ High school } \\
\hline & & & \multicolumn{2}{|c|}{ boys } & \multicolumn{2}{|c|}{ gir/s } & \multicolumn{2}{|c|}{ boys } & \multicolumn{2}{|c|}{ girls } \\
\hline & 198.3 & 1995 & 1983 & 1995 & 1983 & 1995 & 1983 & 1995 & 1983 & 1995 \\
\hline Nome/year & $12 \%$ & $9 \%$ & $16 \%$ & $10 \%$ & $9 \%$ & $4 \%$ & $16 \%$ & $16 \%$ & $9 \%$ & $5 \%$ \\
\hline Once/year & $7 \%$ & $9 \%$ & $8 \%$ & $8 \%$ & $5 \%$ & $5 \%$ & $10 \%$ & $15 \%$ & $5 \%$ & $7 \%$ \\
\hline Few/year & $36 \%$ & $33 \%$ & $43 \%$ & $40 \%$ & $33 \%$ & $33 \%$ & $40 \%$ & $34 \%$ & $29 \%$ & $29 \%$ \\
\hline Once/month & $11 \%$ & $13 \%$ & $9 \%$ & $7 \%$ & $11 \%$ & $12 \%$ & $12 \%$ & $11 \%$ & $11 \%$ & $17 \%$ \\
\hline Few/month & $16 \%$ & $15 \%$ & $12 \%$ & $18 \%$ & $20 \%$ & $21 \%$ & $12 \%$ & $11 \%$ & $22 \%$ & $\mathbf{1 3} \%$ \\
\hline Once/week & $7 \%$ & $10 \%$ & $6 \%$ & $6 \%$ & $11 \%$ & $9 \%$ & $5 \%$ & $8 \%$ & $9 \%$ & $13 \%$ \\
\hline Few/week & $10 \%$ & $13 \%$ & $6 \%$ & $13 \%$ & $11 \%$ & $17 \%$ & $5 \%$ & $6 \%$ & $15 \%$ & $15 \%$ \\
\hline Total number & 2181 & 2358 & 309 & 481 & 293 & 462 & 823 & 596 & 756 & 819 \\
\hline
\end{tabular}

Compared to the 1983 results, the frequency of weekly headaches has risen from $17 \%$ to $23 \%(p=0.032)$. The highest increase was found as an increase in "headaches a few times a week" in boys at elementary school, which doubled from $6 \%$ to $13 \%$.

\section{Headache severity}

Only 201 children, namely $13 \%$ of the boys and $5 \%$ of the girls, reported not having experienced a headache in their lives or in the past year. The low, medium and high headache severity groups consist of $490(21 \%), 1379(59 \%)$ and 288 (12\%) children, respectively. At elementary school, $21 \%$ of the children are within the low and $13 \%$ are within the high headache severity group, while at 
high school these figures are $24 \%$ and $14 \%$, respectively. In addition, $11 \%$ of the boys and $15 \%$ of the girls at elementary school and $7 \%$ of the boys and $17 \%$ of the girls at high school are found in the high headache severity group.

Table 3.4 Percentages of children experiencing specific headache characteristics presented by low, medium and high headache severity group.

\begin{tabular}{|c|c|c|c|c|}
\hline & & Low & Med & High \\
\hline \multirow[t]{5}{*}{ Frequency } & Once or a few a year & $83 \%$ & $42 \%$ & - \\
\hline & Once a month & $17 \%$ & $15 \%$ & - \\
\hline & Few a month & - & $26 \%$ & $=$ \\
\hline & Once a week & - & $9 \%$ & $35 \%$ \\
\hline & Few a week & - & $8 \%$ & $65 \%$ \\
\hline \multirow[t]{2}{*}{ Intensity } & Usual pain in $\mathrm{mm}$, mean (sd) & $24(13)$ & $45(18)$ & $68(14)$ \\
\hline & Worst pain in $\mathrm{mm}$, mean (sd) & $39(21)$ & $63(20)$ & $81(15)$ \\
\hline \multirow[t]{6}{*}{ Duration } & One hour or less & $100 \%$ & $28 \%$ & - \\
\hline & 1 hour $-\frac{1}{2}$ day & - & $30 \%$ & $30 \%$ \\
\hline & $\frac{1}{2}-1$ day & - & $22 \%$ & $34 \%$ \\
\hline & 1-2 days & - & $15 \%$ & $21 \%$ \\
\hline & 2-7 days & - & $4 \%$ & $10 \%$ \\
\hline & $\geq a$ week & - & $1 \%$ & $5 \%$ \\
\hline \multirow[t]{3}{*}{ Headache lacation } & is usually or always unilateral & $15 \%$ & $23 \%$ & $37 \%$ \\
\hline & $\begin{array}{l}\text { usually develops from one side to the } \\
\text { other }\end{array}$ & $8 \%$ & $15 \%$ & $29 \%$ \\
\hline & is usually in the forehead & $73 \%$ & $76 \%$ & $85 \%$ \\
\hline \multirow[t]{5}{*}{ Headache starts } & usually at school & $10 \%$ & $12 \%$ & $17 \%$ \\
\hline & usually at home & $32 \%$ & $25 \%$ & $15 \%$ \\
\hline & usually during the weekend & $9 \%$ & $8 \%$ & $2 \%$ \\
\hline & usually during the week & $24 \%$ & $26 \%$ & $38 \%$ \\
\hline & usually in the morning or upon waking up & $23 \%$ & $43 \%$ & $56 \%$ \\
\hline \multirow[t]{2}{*}{ Headache impacts by } & usually or always interfering with sleep & $1 \%$ & $9 \%$ & $33 \%$ \\
\hline & demanding to do nothing or to lie down & $5 \%$ & $23 \%$ & $52 \%$ \\
\hline \multirow[t]{4}{*}{ Headache is evaluated as } & usually constant & $43 \%$ & $46 \%$ & $61 \%$ \\
\hline & usually or always a tight band & $12 \%$ & $21 \%$ & $45 \%$ \\
\hline & usually severe & $1 \%$ & $6 \%$ & $23 \%$ \\
\hline & usually or always a burden & $1 \%$ & $14 \%$ & $57 \%$ \\
\hline \multirow[t]{5}{*}{ Accompanying symptoms } & usually or always phonophobia & $14 \%$ & $26 \%$ & $41 \%$ \\
\hline & usually or always photophobia & $14 \%$ & $28 \%$ & $52 \%$ \\
\hline & usually or always nausea & $7 \%$ & $24 \%$ & $38 \%$ \\
\hline & usually or always throwing-up & - & $3 \%$ & $6 \%$ \\
\hline & $\begin{array}{l}\text { usually/always aggravation by walking } \\
\text { stairs }\end{array}$ & $22 \%$ & $39 \%$ & $52 \%$ \\
\hline Warning signals & usually precipitate a headache & $19 \%$ & $35 \%$ & $58 \%$ \\
\hline Headache trigger & Stress & $33 \%$ & $48 \%$ & $69 \%$ \\
\hline
\end{tabular}




\section{Headache characteristics}

Table 3.4 is a résumé of differences in headache characteristics in children of the three headache severity groups. Most profound differences are related to the impact of the headache, i.e. interference with sleep and the necessity of doing nothing or lying down, and to the affective evaluation of the headache as severe or as a burden. Accompanying symptoms such as photo- and phonophobia, nausea and throwing-up, and precipitation by warning signals are found more frequently in children with severe headaches. Dizziness $(75 \%)$ and concentration problems $(55 \%)$ form the mostly reported warning signals that children experience in all three groups, followed by visual aura $(37 \%)$, ear ringing $(27 \%)$, and feeling needles (25\%). In addition, other migraine-related symptoms such as unilateral location, pain development from one side of the head to the other, and aggravation by going up or down the stairs are most frequently reported in the group with the highest headache severity. This group also describes headache as a tight band around the head most often, which is a characteristic of tensiontype headache.

Stress was perceived as a headache trigger by $69 \%$ of the children in the high headache severity group. They also mentioned a higher number of stress-related triggers (low: 0.45 (sd 0.73); med: 0.71 (sd 0.90); high: 1.27 (sd 1.13)). More children at high school perceived stress as a headache trigger $(52 \%)$, when compared to children at elementary school $(40 \%)$. Girls $(53 \%)$ more frequently than boys $(40 \%)$, reported at least one stress-related headache trigger.

\section{Headache in the family}

We found that $70 \%$ of the children answered affirmatively on the question whether a member of their family experienced headaches. Children mostly reported that their mother suffered from headaches (low - high: 48-68\%), and $23 \%$ of the fathers as well as the siblings.

\section{Medical consultation}

Overall, 201 children $(9 \%)$ with headaches in the past year had consulted a physician because of headache (low: $2 \%$, medium: $7 \%$, high: $30 \%$ ). Children who had required medical consultation were asked to state their doctor's response in an open-ended answer format. "I don't remember" was answered most frequently in the low $(35 \%)$ and medium $(41 \%)$ headache severity group. In the high headache severity group, the advice most often given by the physician was to check whether glasses are needed (26\%) and to take pain medication (24\%). 
Still, $13 \%$ could not reproduce what their doctor had told them. "Your headaches are migraine" was recorded by $17 \%, 14 \%$ and $10 \%$ of the children in the low, medium and high headache severity group, who had consulted their physician. Keeping a headache diary was suggested only 4 times and only in case of severe headaches.

\section{School absence}

School absence due to headache was reported by $41 \%$ of all children with headaches in the past year.

In the group with the highest headache severity $64 \%$ of the children reported having been absent of school due to headache, compared to $45 \%$ in the medium and $17 \%$ in the lowest headache severity group. A higher percentage of children at elementary school $(52 \%)$ reported absence, when compared to children at high school ( $34 \%)$. There was no difference between boys and girls.

\section{DISCUSSION}

Almost all schoolchildren reported the experience of one or more headaches in the past year. Half of the sample reported headaches of at least once a month, including $23 \%$ that complained of headaches once a week or more often. Since 1983, the prevalence of weekly headaches in school children between the age of 10 and 17 years has risen with $6 \%$, and the rise was found in particular as an increase in a few headaches a week at elementary school (10-12 years of age). The increase in headache prevalence that has been reported in young children at school entry in Finland ${ }^{10}$ was thus confirmed in the Dutch situation focusing on school children.

Headache severity groups were constructed based on criteria of frequency, intensity and duration, making group differences on these variables imperative. Clinically relevant differences however, were found on all other headache characteristics. Migraine-related characteristics, such as unilateral headache pain, headache onset in the morning or upon waking up, accompanying symptoms such as nausea, vomiting and photo- and phonophobia, and precipitating warning signals were found most frequently in the high headache severity group. This comes up to expectations, because a higher pain intensity and duration define migraine. ${ }^{19}$ Nevertheless, the sensation of headache as a tight band around the head, which is typical for tension-type headache, is also found most frequently in the high severity group. 
Stress is reported as the most frequent precipitating factor for headache, both in adults ${ }^{20,21}$ and in children. ${ }^{3,5,22-24}$ We found that $69 \%$ of the children with the most severe headaches perceived stress as a headache cause. The importance of research on stress and headache remains eminent. ${ }^{21}$

School absence in the past year due to headache was reported by $19 \%, 48 \%$ and $64 \%$ of the children in the low, medium and high headache severity groups, respectively. These results are higher than in other studies. Using prospective class registration, Collin et al. ${ }^{25}$ found that only 3 to $7 \%$ of $5-14$ year old pupils reported school absence related to headache, in two 12-week periods. In a telephone survey, Linet et al. ${ }^{26}$ found $12 \%$ of $12-17$ year olds reporting absence from school or work because of headache. Comparison of results is complicated because no differences between headache severity groups have been made in other studies. In addition, we rely solely on the report of the child and have no information on the frequency or duration of absence due to headache. Consultation of a (family) physician because of headaches $(9 \%$ ) was comparable to other studies. ${ }^{5,26}$ The highest percentage of medical consultation (30\%) was found in the children with the most severe headaches. This is in line with Metsähonkala et al. ${ }^{27}$, who reported that in a group of children with migraine. use of health care services was highest in children with the most frequent attacks and the highest number of missed school days.

A few methodological issues need to be addressed, with respect to the sample, the questionnaire and headache assessment. In addition, we need to stress the cross-sectional nature of our study, which limits conclusions about causal relationships.

Our study involves a large sample of schoolchildren that represents the population at hand. No attempt has been made to collect data of children who were absent at the day of assessment or who did not give consent. Previously, no differences have been found between participants and absent subjects with regard to headache complaints. ${ }^{3}$ This leads us to hypothesize that the distribution of headache frequency in the sample present fits the absent population. If that hypothesis were not true, one would expect an underestimation of headache frequency in our sample, which is at least a conservative approach.

Self-report is usually considered the gold standard in pain assessment, because it accounts for the subjective nature of pain. ${ }^{28}$ Behavioral and physiological components of pain 29 have not been included in the measurement and assessment of pediatric headache so far. Studies in children have shown little differences in headache report between child, parent and physician. ${ }^{30}$ 
Test-retest studies of headache questionnaires in children have shown consistent reliability. ${ }^{31,32}$ Despite that, self-report measures in cross-sectional studies are retrospective and children are asked to recall headache frequency and characteristics over the past month or year. Recall errors may have occurred.

Comparison of current and previous results ${ }^{3}$ in our study can hardly be hampered by differences with respect to the questionnaires used. Layout differences are merely typographical. Although items were added in our study to update information on headache characteristics, we only compared the item on headache frequency, which was exactly alike, in our statistical analysis. Method of administration in the classroom setting was comparable. However, differences between the two cities may encompass environmental differences related to headache, such as industrial pollution. In addition, cultural differences related to complain behavior might contribute to difficulties in comparison. In addition, talking about and reporting headaches may have become socially more acceptable over time, as has been suggested by Sillanpää and Anttila. ${ }^{10}$ Seasonal differences influencing headache experience may exist. Our study was performed in winter (November to January), while the previous study was conducted in spring-time (personal communication).

In both studies, as in other population-based studies (table 3.2), no physical examinations have taken place in order to classify headache or to explicitly exclude secondary headaches. However, the studies are focused on primary headaches and the definition of primary headache is used from a lay perspective. ${ }^{2}$ Using headache severity as a classification method, instead of focusing on a well-defined population of migraine and/or tension-type headache patients, sheds light on the total problem of headache experienced by school children.

In conclusion, the percentages of children reporting headaches have slightly increased in a decade, from $88 \%$ in 1983 to $91 \%$ in 1995 . The small size of increase may be explained as a ceiling effect. Yet, the shift that has been found towards the report of more frequent (i.e. weekly) headaches is remarkable.

Several questions remain unanswered. Will the increase towards more weekly headaches continue over time, why is the increase found specifically in children of a younger age, what contributes to the increase in frequent headaches and, most importantly, what preventive measures can be taken to reduce the prevalence of frequent headaches? 


\section{REFERENCES}

1. King NJ. Sharpley CF. Headache activity in children. Paediatr. Child Health 1990:26:50-54.

2. Kristjánsdóttir 6 , Wahlberg $V$. Saciademagraphic differences in the prevalence of selfreported headache in Icelandic school-children. Headache 1993:33:276-280.

3. Passchier J. Orlebelke JF. Headaches and stress in schoolchildren: an epidemiological study. Cephalalgia 1985;5:167-176.

4. Small P, Waters WE. Headache and migraine in a comprehensive school. In: Waters WE (Ed.). the epidemiology of migraine, Bracknell: Boehringer Ingelheim, 1974, p. 59-67.

5. Egermark-Eriksson I. Prevalence of headache in Swedish schoolchildren. Acto Paediatt. Scand. 1982:71:135-140.

6. Sillanpäa M. Prevalence of headache in prepuberty. Headache 1983a;23:10-14.

7. Sillanpää $M$. Changes in the prevalence of migraine and other headaches during the first seven schaol years. Headache 1983b;23:15-19.

8. Larsson B. The rolle of psychological, health-behaviour and medical factors in adolescent headache. Dev. Med. Child Neural. 1988:30:616-625.

9. Carlsson J. Prevalence of headache in schoolchildren: relation to family and school factors. Acta Paediatr. 1996:85:692-696.

10. Sillanpäa M. Anttila P. Increasing prevallence of headache in 7 -year-old schoolchildren. Headache 1996:36:466-470.

11. Larsson B, Daleflod B, Häkanson L, Melin L. Therapist-assisted versus self-help relaxation treatment of chronic headaches in adolescents: a school-based intervention. $\mathcal{T}$. Child Psychol. Psychiat, 1987:28:127-136.

12. McGrath PJ, Humphreys $P$, Goodman JT, Keene D. Firestone $P$, Jacob $P$, Cunningham $S J$. Relaxation prophylaxis for childhood migraine: a randamized placebo-contralled trial. Dev. Med. Child Neurol 1988;30:626-631.

13. Waters WE. Community studies of the prevalence of headache. Headache 1970;1:178-186

14. Moss G, Waters WE. Headache and migraine in a giris' grammar school. In: Waters WE (Ed.). The epidemiology of migraine. Bracknell: Boehringer Ingelheim 1974, p. 49-58.

15. Abu-Saad H. Assessing children's responses to pain. Pain 1984:19:163-171.

16. Huijer Abu-Saad H. Toward the development of an instrument to assess pain in children: Dutch study. In: Tyler DC and Krane EJ, editors. Advances in Pain Research Therapy. Vol. 15. New York: Raven Press Ltd, 1990, p. 101-106.

17. Huijer Abu-Saad H, Kroonen E. Halfens R. On the development of a multidimensional Dutch pain assessment tool for children. Poin 1990:43:249-256.

18. McGrath PJ, Humphreys P., Keene D, Goadman JT, Lascelles MA, Cunningham SJ, Firestone, $P$. The efficacy and efficiency of a self-administered treatment for adolescent migraine. Pain 1992;49:321-324.

19. IHS: Headache classification committee of the International Headache Society. Classification and diagnostic criteria for headache disorders, cranial neuralgias and facial pain. Cephalalgia 1988:8 (suppl. 7):1-96.

20. Andrasik F, Passchier, J. Tension-type headache, cluster headache, and miscellaneous headaches. Psychological aspects. In: Olesen J. Tfelt-Hansen P, Welch KMA. The headaches. New Yorki Roven Press. 1993. p. 489-492.

21. Passchier J, Andrasik F. Migraine. Psychological factors. In: Olesen J. Tfelt-Hansen P. Welch KMA. The headaches. New York: Raven Press. 1993, p. 233-240. 
22. Jacobides 6M. Migraine in children and adolescents. In: Rose FC. Advances in migraine research and therapy. New York: Raven Press, 1982, p. 39-44

23. Maratos J. Wilkinson M. Migraine in children: a medical and psychiatric study. Cephalalgia $1982: 2: 179-187$.

24. Metsähonkala $L$, Sillanpäa $M$, Tuominen J. Social environment and headache in 8-to 9-yearold children a fallow-up study. Headache 1998:38:222-228.

25. Collin C. Hockaday JM, Waters WE. Headache and schoal absence. Arch. Dis. Child. 1985:60:245-247.

26. Linet MS, Stewart WF, Celentano DD, Ziegler D, Sprecher M. An epidemiolagic study of headache among adolescents and young adults. JAMA1989;261:2211-2216.

27. Metsáhonkala $L$, Sillanpäa $M$. Tuaminen $J$. Use of health care services in childhood migraine. Headache 1996:36:423-428.

28. Merskey $H$ (Ed.). Classification of chronic pain: desccriptians of chronic pain syndromes and pain terms. Pain 1986: suppl. 3:5217.

29. McGrath PJ, Unruh AM. Pain in children and adolescents. Amsterdam: Elsevier, 1987.

30. Andrasik F. Burke EJ, Attanasio V. Rosenblum EL. Child, parent and physician reports of a child's headache pain: Relationships prior to and following treatment. Headache. 1985:11:421-25.

31. Thompsan JK, Collins FL. Reliability of headache questionmaire data. Headache 1979;19:97101.

32. Waters WE (Ed.). The epidemiology of migraine. Bracknell: Boehringer Ingelheim, 1974. 


\section{Chapter 4}

Coping and quality of life in relation to headache in Dutch schoolchildren

Bandell-Hoekstra I.E.N.G., Huijer Abu-Saad H, Passchier J, Frederiks CMA, Feron FJM, Knipschild P. Coping and quality of life in relation to headache in Dutch schoolchildren. European Journal of Pain 2002; 6:315-321. 


\section{ABSTRACT}

Aim of this study is to describe the Quality of Life and pain coping strategies of school children in relation to headache severity. We conducted a cross-sectional study in 2815 children between the age of 9 and 17 years, who filled out Waters' Headache Questionnaire (WHQ), the Pediatric Pain Assessment Tool (PPAT), the Quality of Life Headache in Youth (QLH-Y) questionnaire and the Pain Coping Questionnaire (PCQ) in the class-room setting.

Weekly headaches were reported by $22 \%$ of the sample. Low, medium, and high headache severity groups were constructed, based on headache frequency, duration and intensity criteria.

Results show that children with the highest headache severity report the lowest Quality of Life in general and the lowest Quality of Health, as well as the most problems with regard to physical functioning, impact of headache on daily and leisure activities, physical symptoms other than headache, and social functioning at home. With regard to using pain coping strategies, children with the most severe headaches seek more social support, they internalize and externalize more, they use less behavioral and cognitive distraction techniques, and seek information less. 


\section{INTRODUCTION}

Only a few studies so far, have focused on headache-related Quality of Life (QoL). If so, the study population is usually confined to adult migraine sufferers, who report a clearly diminished QoL in comparison to the general population, 1,2 even in between attacks, when they are compared with headache-free controls. ${ }^{3}$ The development of the Quality of Life Headache in Youth (QLH-Y) questionnaire ${ }^{4}$ has provided the impulse to call attention upon QoL in children and adolescents. A study on adolescents with chronic headache and migraine showed that changes in actual headache presence change satisfaction with life in general and satisfaction with health. ${ }^{5}$

QoL is a concept that encompasses a broad range of physical and psychological characteristics and limitations that describe an individual's ability to function, and the satisfaction derived from doing so. ${ }^{6}$ Health-related QoL is affected by physical health, psychological state, level of independence, social relationships, and relationships to salient features of the environment. ${ }^{7}$ The relationship between headache and QoL is complex. We assume a bi-directional relationship, in which headache influences QoL and QoL influences headache. Coping strategies act upon both. ${ }^{8}$

Coping is an intentional cognitive and behavioral effort to manage stress, with a focus on the problem that causes stress or on emotion-regulation. ${ }^{9,10}$ Children perceive stress as an important trigger of headache. ${ }^{11,12}$ Assessment of coping strategies to manage stress needs to be the focus of studies aiming at the description of this trigger. However, the headache pain itself can also be viewed as a stressor that needs to be dealt with. Hence, assessment of pain coping strategies needs to be addressed. Children with recurrent headaches mostly prefer taking medication or lying down, to cope with the pain. Moreover, they use distraction, relaxation, seeking social support, problem solving, maintaining a future orientation, remaining positive, wishful thinking and becoming helpless. ${ }^{13}$ Since $23 \%$ of $10-17$ year old school children complain of weekly headaches, ${ }^{12}$ focus on recurrent headaches in the general, non-referred population of schoolaged children is indicated. Studies on QoL and pain coping in relation to headache in this population are lacking. Aim of the current study was to describe Quality of Life and pain coping strategies in the general population of 9 to 17 year old school children. To this end, we classified the population according to headache severity and compared Quality of Life and pain coping measures of children with low, medium and high headache severity. 


\section{METHODS}

\section{Subjects and study procedures}

We canducted a cross-sectional study and administered self-report questionnaires to elementary and high school pupils, in the class-room setting. Subjects and sampling methods as well as study procedures have been described elsewhere. ${ }^{12}$ School children aged between 9 and 17 years form the population of the current study, which means that with regard to subjects, data of the three highest grades of elementary school were included in the current analysis. Approximately $40 \%$ of the children at elementary schools in the City, as well as $20 \%$ of the high school pupils in the City and region of Maastricht, have been included.

\section{Measures}

Assessment of headache severity was based on an adapted version of Waters' Headache Questionnaire (WHQ), 11,14,15 which was completed with the 0-100 millimeter (mm) Visual Analogue Scale (VAS) on usual and worst pain intensity of the Pediatric Pain Assessment Tool (PPAT). ${ }^{16-18}$

Quality of Life (QoL) was assessed using the Quality of Life Headache in Youth (QLH-Y) questionnaire. ${ }^{4}$ The QLH-Y covers two $0-100 \mathrm{~mm}$ visual analogue scales on QoL in general and Quality of Health, and consists of 69 items comprising 4 sub-domains (psychological functioning, physical functioning, social functioning and functional status). The subscales that encompass the sub-domains of the QLH-Y (see table 4.3) were more or less confirmed by principal component factor analysis using oblique rotation. Cronbach's alphas were between 0.77 and 0.97. After a pilot-study on the length and difficulty of the questionnaires, the wording of a few items of the QLH-Y were simplified.

Pain coping was assessed using the Pain Coping Questionnaire (PCQ). ${ }^{19}$ The 39 items cover 8 subscales, concerning information seeking, problem solving, seeking social support, positive self-statements, behavioral distraction, cognitive distraction, externalizing and internalizing or catastrophizing. The PCQ was translated into Dutch using the forward-backward method.

Factor analysis of the PCQ (maximum likelihood procedure using oblique rotation) confirmed the subscales that were presented by Reid, ${ }^{19}$ but did not clearly differentiate between higher-order sub-domains. Cronbach's alphas of the PCQ subscales were between 0.78 and 0.87 .

A test-retest (after 4 weeks) showed moderate to high reproducibility of all measures. 


\section{Data analysis}

Questionnaires of children younger than 9 years of age or aged 18 years and older were excluded, in addition to questionnaires with missing values on headache frequency, intensity or duration.

Headache frequency was described for the whole sample, but the small group of children that reported no experience of a headache ever in their lives or in the past year were not taken into account in further analysis. The sample was divided into three groups, based on headache severity. The high headache severity group consisted of children who combined a headache frequency of once a week or more often with a usual headache intensity of at least $50 \mathrm{~mm}$ on the VAS, and a headache duration usually lasting more than an hour. The low headache severity group included only children with a headache frequency of once a month or less, a usual headache intensity of less than $50 \mathrm{~mm}$ on the VAS. and a usual headache duration of one hour maximum. The medium headache severity group included all other children.

Pain coping and Quality of Life were inventoried using descriptive statistics. The means and $95 \%$ confidence intervals were described for each subscale of the questionnaires QLH-Y and PCQ, comparing subjects in the low, medium and high headache severity group. We conclude that there is a difference between groups, if the $95 \%$ confidence intervals do not overlap.

\section{RESULTS}

\section{Sample}

Of the 3300 children in the participating school classes, $2871(87 \%)$ actually filled out the questionnaires. Non-participation was mainly due to absence due to illness $(6 \%)$, the child's refusal $(4 \%)$, and no permission of the parents $(3 \%)$. Of the filled out questionnaires, $2815(98 \%)$ were available for analysis: 19 were omitted because the child's age was below 9 years or 18 years or older and 37 because of missing values on headache frequency, intensity or duration items. Mean age of the sample was 12.6 (sd 2.0). The distribution of boys and girls was equal for elementary school: at high school more girls participated $(58 \%)$. Table 4.1 presents the results of headache frequency in total as well as subdivided according to gender and age. 
Table 4.1 Headache frequency: percentages of boys and girls in each grade of elementary and high school.

\begin{tabular}{|c|c|c|c|c|c|c|c|c|c|c|c|c|c|c|c|}
\hline Gender & & & & boys & & & & & & & girls & & & & \\
\hline School type & & stment & & & High & choo & & & ment & & & High & schoo & & \\
\hline Grodede & 6 & 7 & 8 & 1 & 2 & 3 & 4 & 6 & 7 & 8 & 1 & 2 & 3 & 4 & total \\
\hline None/year & $13 \%$ & $10 \%$ & $10 \%$ & $17 \%$ & $17 \%$ & $15 \%$ & $13 \%$ & $6 \%$ & $3 \%$ & $4 \%$ & $9 \%$ & $3 \%$ & $3 \%$ & $6 \%$ & $9 \%$ \\
\hline Once/year & $13 \%$ & $8 \%$ & $7 \%$ & $11 \%$ & $15 \%$ & $17 \%$ & $14 \%$ & $9 \%$ & $5 \%$ & $5 \%$ & $8 \%$ & $9 \%$ & $6 \%$ & $3 \%$ & $9 \%$ \\
\hline Few/year & $39 \%$ & $40 \%$ & $40 \%$ & $33 \%$ & $37 \%$ & $33 \%$ & $33 \%$ & $31 \%$ & $29 \%$ & $37 \%$ & $31 \%$ & $31 \%$ & $24 \%$ & $32 \%$ & $33 \%$ \\
\hline Once/month & $10 \%$ & $7 \%$ & $7 \%$ & $10 \%$ & $7 \%$ & $13 \%$ & $16 \%$ & $9 \%$ & $9 \%$ & $15 \%$ & $18 \%$ & $16 \%$ & $15 \%$ & $21 \%$ & $12 \%$ \\
\hline Few/month & $10 \%$ & $19 \%$ & $17 \%$ & $13 \%$ & $8 \%$ & $9 \%$ & $14 \%$ & $18 \%$ & $24 \%$ & $18 \%$ & $9 \%$ & $13 \%$ & $17 \%$ & $14 \%$ & $15 \%$ \\
\hline Once/week & $4 \%$ & $7 \%$ & $5 \%$ & $8 \%$ & $9 \%$ & $8 \%$ & $9 \%$ & $7 \%$ & $10 \%$ & $8 \%$ & $10 \%$ & $12 \%$ & $17 \%$ & $14 \%$ & $9 \%$ \\
\hline Few/week & $10 \%$ & $10 \%$ & $15 \%$ & $9 \%$ & $6 \%$ & $4 \%$ & - & $22 \%$ & $21 \%$ & $14 \%$ & $15 \%$ & $16 \%$ & $18 \%$ & $10 \%$ & $13 \%$ \\
\hline number & 211 & 238 & 242 & 201 & 111 & 186 & 99 & 246 & 234 & 226 & 244 & 207 & 246 & 124 & 2815 \\
\hline
\end{tabular}

"elementary school - grade 6: 9-10 yrs, grade 7: 10-11 yrs, grade 8: 11-12 yrs:

high school - grade 1: 12-13 yrs, grade 2: 13-14 yrs, grade 3: 14-15 yrs, grade 4: 15-16 yrs

Weekly headaches are reported by $9-20 \%$ of the boys in the different grades, and by $22-35 \%$ of the girls.

Only $242(9 \%)$ children reported not having experienced a headache ever in their life or in the past year. The low, medium and high headache severity groups consist of $605(22 \%), 1626(58 \%)$ and $342(12 \%)$ children, respectively (see table 4.2).

Table 4.2 Number and percentages of children that were included in the low, middle and high headache severity, by inclusion criterion.

\begin{tabular}{|c|c|c|c|}
\hline Group & n & Inclusion criteria & $\%$ \\
\hline No & 242 & No headaches ever in life or in the previous year & 8.6 \\
\hline Low & 605 & Headache frequency $\leq$ once a month * intensity $<50^{*}$ duration $\leq 1 \mathrm{hr}$ & 21.5 \\
\hline \multirow[t]{7}{*}{ Medium } & 1626 & Headache frequency $\leq$ once a month * intensity $<50 *$ duration $>1 \mathrm{hr}$ & 17.3 \\
\hline & & Headache frequency $\leq$ once a month * intensity $\geq 50^{*}$ duration $\leq 1 \mathrm{hr}$ & 5.0 \\
\hline & & Headache frequency $\leq$ once a month * intensity $\geq 50$ * duration $>1 \mathrm{hr}$ & 10.6 \\
\hline & & Headache frequency $=a$ few times a month & 14.8 \\
\hline & & Headache frequency $\geq$ once a week * intensity & 2.7 \\
\hline & & Headache frequency $\geq$ once a week * intensity $<50 *$ duration $>1 \mathrm{hr}$ & 3.9 \\
\hline & & Headache frequency $\geq$ once a week * intensity $\geq 50^{*}$ duration $\leq 1 \mathrm{hr}$ & 3.5 \\
\hline High & 342 & Headache frequency $z$ once a week $k^{*}$ intensity $\geq 50$ * duration $>1 \mathrm{hr}$ & 12.1 \\
\hline
\end{tabular}




\section{Quality of Life}

Table 4.3 shows the differences between low, medium and high headache severity on the QLH-Y subscales. When compared to children with a low or medium headache severity, children with a high headache severity experience more stress, less harmony and vitality, more fatigue, more depression, and more somatic symptoms other than headache. They rate their functioning at home lower and experience a higher impact of headache on daily and leisure activities. There is no significant difference between the three groups with regard to social interaction with peers and with siblings, functioning at school, and optimism about the future. Children in both the high as well as the medium headache severity group have lower scores on cheerful mood, than children in the low headache severity group.

Table 4.3 Mean and 95\% confidence intervals of QLH-Y subscales presented by low, medium and high headache severity group.

\begin{tabular}{llccc}
\hline Sub-domain & Subscale & Low & Medium & High \\
Psychological Stress & $0.64[0.52-0.76]$ & $1.12[1.01-1.22]$ & $2.19[1.85-2.54]$ \\
functioning & Harmony + vitality & $6.06[5.69-6.42]$ & $4.99[4.77-5.21]$ & $2.95[2.54-3.36]$ \\
& Fatigue & $0.56[0.46-0.66]$ & $1.02[0.94-1.10]$ & $1.79[1.57-2.02]$ \\
& Depression & $0.65[0.54-0.76]$ & $0.85[0.77-0.93]$ & $1.84[1.58-2.10]$ \\
& Cheerful mood & $3.23[3.05-3.41]$ & $2.03[1.93-2.14]$ & $1.76[1.55-1.98]$ \\
& Optimism & $1.98[1.82-2.14]$ & $2.03[1.93-2.14]$ & $1.76[1.55-1.98]$ \\
Physical & Somatic symptoms & $1.10[0.96-1.25]$ & $1.89[1.76-2.02]$ & $3.66[3.27-4.05]$ \\
functioning & & & & \\
Social & Functioning at home & $3.05[2.91-3.19]$ & $2.66[2.57-2.75]$ & $2.33[2.12-2.53]$ \\
functioning & Functioning at school & $3.02[2.87-3.17]$ & $2.84[2.75-2.94]$ & $2.59[2.38-2.81]$ \\
& Social interaction & $2.65[2.52-2.78]$ & $2.66[2.58-2.75]$ & $2.53[2.34-2.73]$ \\
& with peers & & & \\
& Social interaction & $2.21[2.05-2.38]$ & $2.05[1.95-2.15]$ & $2.00[1.79-2.21]$ \\
& with siblings & & & \\
Functional & Impact an daily life & $1.15[0.85-1.45]$ & $2.18[1.97-2.38]$ & $3.49[3.05-3.93]$ \\
status & and leisure activities & & & \\
\hline
\end{tabular}

a subjects without headache in the week previous to completion of the QLH-Y were excluded from analysis on this sub-domain

With regard to the sub-domains of the QLH-Y, presented in table 4.4, we found that children with the highest headache severity report the lowest Quality of Life in general, Quality of health, psychological functioning, functional status, and physical symptoms other than headache. Children in the low headache severity group experience the highest Quality of Life and children in the medium headache severity group report Quality of Life scores in between. 
When compared to the low headache severity group, social functioning is lower in the medium as well as the high headache severity group.

Table 4.4 Mean and $95 \%$ confidence intervals of QLH-Y sub-dornains presented by low, medium and high headache severity group. Negative scores have been recaded, i.e. high scores mean high QDL.

\begin{tabular}{lccc}
\hline & Low & Medium & High \\
Quality of Life & $76[74-78]$ & $71[70-72]$ & $67[64-70]$ \\
Quality of Health & $76[74-78]$ & $68[66-69]$ & $59[56-63]$ \\
Psych. functioning & $63.24[62.60-63.87]$ & $61.05[60.61-61.48]$ & $55.44[54.50-56.38]$ \\
Functional status & $40.34[40.00-40.69]$ & $39.77[39.55-39.98]$ & $38.78[38.35-39.21]$ \\
Physical symptoms & $25.89[25.41-25.77]$ & $25.15[25.03-25.28]$ & $23.67[23.30-24.03]$ \\
Social functioning & $10.95[10.50-11.39]$ & $10.17[9.91-10.44]$ & $9.48[8.89-10.08]$ \\
\hline
\end{tabular}

"subjects without headache in the week previous to completion of the QLH-Y were excluded from analysis on this sub-domain

\section{Pain coping strategies}

Results of the differences between the low, medium, and high headache severity groups in using pain coping strategies for dealing with headache, are reported in table 4.5. All three groups differ in seeking social support, using behavioral distraction and cognitive distraction, internalizing/catastrophizing and externalizing. A higher headache severity means seeking social support more, but using less distraction techniques and more catastrophizing and externalizing. There is no difference between the three groups in using positive selfstatements. Children in the medium and high headache severity group use more problem solving strategies than children with low headache severity. Children in the high headache severity group use less information seeking strategies.

Table 4.5 Mean and $95 \%$ confidence intervals of PCQ subscales presented by low, medium and high headache severity group.

\begin{tabular}{lccc}
\hline & \multicolumn{1}{c}{ Low } & Med & High \\
Problem solving & $1.90[1.83-1.96]$ & $2.07[2.03-2.11]$ & $2.21[2.11-2.31]$ \\
Information seeking & $2.90[1.28-1.36]$ & $2.69[1.35-1.40]$ & $2.36[1.45-1.58]$ \\
Positive self statements & $1.28[2.20-2.36]$ & $1.36[2.20-2.30]$ & $1.59[2.04-2.23]$ \\
Seeking social support & $1.87[1.81-1.94]$ & $2.07[2.02-2.11]$ & $2.22[2.13-2.31]$ \\
Cognitive distraction & $2.90[2.82-2.98]$ & $2.69[2.64-2.74]$ & $2.36[2.26-2.45]$ \\
Behavioral distraction & $2.54[2.47-2.61]$ & $2.30[2.26-2.34]$ & $2.09[2.00-2.18]$ \\
Internalizing & $1.50[1.45-1.54]$ & $1.78[1.75-1.82]$ & $2.34[2.24-2.44]$ \\
Externalizing & $1.28[1.24-1.32]$ & $1.36[1.33-1.39]$ & $1.59[1.51-1.67]$ \\
\hline
\end{tabular}




\section{DISCUSSION}

Headache severity and QoL were found to be negatively rellated. Children with at least weekly headaches with a mean duration of more than an hour and a mean pain intensity of at least $50 \mathrm{~mm}$ on a $0-100 \mathrm{~mm}$ VAS, experience the lowest QoL in all sub-domains. They report the lowest $Q \circ L$ in general and the lowest $Q 0 H$, and rate their psychological functioning, functional status, and physical symptoms other than headache worse than children with medium or low headache severity. Children with medium and high headache severity equally rated their social functioning worse than children with low headache severity. Within the sub-domain psychological functioning, children with the highest headache severity specifically experienced more stress, less harmony and vitality, more fatigue, and more depression.

Headache severity is related to the use or disuse of coping strategies. Children with the most severe headaches seek more social support, by talking to someone about how they feel. They internalize and externalize more. Internalizing means that they worry more and keep on thinking about the pain, and that it will never stop. Externalizing means that they express their hurt by yelling, cursing or getting mad or mean. They use less behavioral and cognitive distraction techniques, such as doing something funny or active, or trying not to think about it. They also seek information (asking questions about the headache) less.

Before drawing conclusions, several features of our study design need to be discussed. First of all, the cross-sectional nature of our study does not permit to draw firm conclusions about the direction or the causality of the relationships, between headache severity and QoL, headache severity and pain coping strategies, and pain coping and QoL. More severe headaches may influence QoL. But a low score on elements of QoL, such as stress, depression, and difficulties with regard to social functioning, may contribute to headache problems as well. A more prospective study design in adolescents, showed that a high score on experienced stress increases the impact of headache on psychological functioning and satisfaction with life. ${ }^{20}$ Pain coping strategies may be influenced by previous pain experiences, parental coping and actions towards the child, role modeling, perceived pain cause, self-efficacy and so on. ${ }^{8}$ In addition, pain coping strategies have an impact on pain severity. Good or healthy pain coping strategies, however, are difficult to distinguish from bad coping and are determined by individual differences, type of pain and underlying disease, nature of the pain (i.e. acute, chronic), the distinction between coping style (trait) and specific coping strategies (state), and short-term adjustment and long-term effects. ${ }^{19}$ In addition, the influence of person-specific and situation- 
specific variables on headache, pain coping and QoL still needs to be unraveled. A literature overview and a conceptual model on headache, coping, QoL and influencing variables has recently been published. ${ }^{8}$

Second, the QLH-Y and PCQ both focus on patient populations. The QLH-Y has been developed for adolescents with chronic headaches or migraine. It is, however, a generic QoL measurement scale, in which only the subscale "functional status" refers to headache. Item reduction and factor analysis was conducted in a sample of healthy school children. ${ }^{5}$ The inclusion of younger children in our study did not result in problems with construct validity. The PCQ shows different higher order factor structures within a healthy population, and within a sample of children with recurrent pain, including headache. ${ }^{19}$ The Danish translation of the PCQ, tested within healthy children, resulted in 7 subscales instead of $8 .{ }^{21}$ More studies on the PCQ. including a broad international range to allow for cultural and translation differences, are thus warranted.

Third, we focused on primary headaches using headache severity as a classification method, instead of focusing on a well-defined population of migraine and/or tension-type headache patients. Our approach sheds light on the total problem of headache in school children, but studies on specific patient populations are needed as well.

We conclude with stressing the importance of QoL, and the challenge of pain coping, in studies on pediatric headache. Children with the highest headache severity score significantly lower on Quality of Life, compared to their peers with less severe headaches, and they use pain coping strategies differently. This confirms the need for studies on headache treatment in children.

We need to know more about QoL and pain coping strategies in relation to headache. Prospective studies would provide more information about the direction of relationships. In addition, QoL and pain coping strategies should not only be included in assessment studies but in treatment studies as well.

\section{REFERENCES}

1. Michel P. Dartigues JF, Lindoulsi A, Henry P. Loss of productivity and Quality of Life in migraine sufferers among French workers: results from the GAZEL cohort. Headache 1997:37:71-78.

2. Osterhaus JT, Townsend RJ, Gandek B, Ware JE. Measuring the functional status and wellbeing of patients with migraine headache. Headache 1994;34:337-343.

3. Dahlöf $C G H$, Dimenäs E. Migraine patients experience poorer subjective well-being/quality of life even between attacks. Cephalalgia 1995;15:31-36. 
4. Langeveld JH, Koot HM, Loonen MC, Hazebroek-Kampschreur AAJM, Passchier J. A quality of life instrument for adolescents with chronic headache. Cephalalgio 1996:16:183-196.

5. Langeveld $J H$, Koot HM, Passchier J. Headache intensity and quality of life in adolescents. How are changes in headache intensity in adolescents related to changes in experienced quality of life? Headoche 1997:37:37-42.

6. Walker SR, Rosser RM, eds. Quality of Life: Assessment and Application. Lancaster: MTP Press: 1988.

7. WHOQOL Group. Measuring Quality of Life: The Development of the World Health Organization Quality of Life Instrument (WHOQQL). Geneva: WHO; 1993.

8. Bandell-Haekstra, I., Huijer Abu-Saad, H., Passchier, J., Knipschild, P. Recurrent headache, coping and Quality of Life in children: a review. Headache 2000;40:357-370.

9. Lazarus RS. Emation and Adaptation. New York: Oxford University Press: 1991.

10. Lazarus RS, Folkman S. Stress, Appraisal and Coping. New York: Springer: 1984.

11. Passchier J, Orlebeke JF. Headaches and stress in schoolchildren: an epidemiological study. Cephalalgia 1985;5:167-176.

12. Bandell-Hoekstra IENG, Huijer Abu-Saad H, Passchier J, Frederiks CMA, Feron FJM, Knipschild P. Prevalence and characteristics of headache in Dutch schoolchildren. European Journal of Pain 2001:5:145-153.

13. Spirito A, Stark LJ, Williams $C$. Development of a brief coping checkllist for use with pediatric populations. Journal of Pediatric Psychology 1988:13:555-574.

14. Waters WE. Community studies of the prevalence of headache. Headache 1970:1:178-186.

15. Moss G. Waters WE. Headache and migraine in a girls grammar school In: Waters WE (Ed.). The epidemiology of migraine. Bracknell: Boehringer Ingelheim, 1974, p. 49-58

16. Abu-Saad H. Assessing children's responses to pain. Pain 1984:19:163-171.

17. Huijer Abu-Saad $H$. Toward the development of an instrument to assess pain in children: Dutch study. In: Tyler DC and Krane EJ, editars. Advances in Pain Reseanch Therapy. Vol. 15. New York: Raven Press Ltd, 1990: 101-106.

18. Huijer Abu-Saad H, Kroonen $E$, Halfens R. On the development of a multidimensional Dutch pain assessment tool for children. Pain 1990;43:249-256.

19. Reid GJ, Gilbert CA, MCGrath PJ. The Pain Coping Questionnaire: preliminary validation. Pain $1998 ; 76: 83-96$.

20. Langeveld JH, Koot HM, Passchier J. Do experienced stress and trait negative affectivity moderate the relationship between headache and Quality of Life in adolescents? Journal of Pediatric Psychology 1999:24:1-11.

21. Thastum M, Zachariae $R$, Scholer M. Herlin, T. A Danish adaptation of the Pain Coping Questionnaire for children: preliminary data concerning reliability and validity. Acto Paediatrica 1998:88:132-138. 
72

Chapter 4 


\section{Chapter 5}

The occurrence of recall bias in pediatric headache: a comparison of questionnaire and diary data

Brink $M$ van den, Bandell-Hoekstra ENG. Huijer Abu-Saad $H$. The occurrence of recall bias in pediatric headache: a comparison of questionnaire and diary data. Headache 2001:41:11-20. 


\section{ABSTRACT}

Purpose of this study was to investigate whether children and adolescents can recall prior headache complaints accurately and to study whether age, gender, headache severity, preferred coping strategies, depression, somatization and trait anxiety are related to recall errors, causing recall bias.

A retrospective headache questionnaire and a prospective four week headache diary was filled in by 181 children 9 to 16 years of age who experienced at least weekly headaches. In addition, several other questionnaires were administered, measuring coping strategies, depression, somatization and trait anxiety. Headache frequency, intensity and duration, as scored on the questionnaire and the diary, were compared using Wilcaxon's tests. Regression analyses were performed to study whether age, gender, headache severity, preferred coping strategies, depression, somatization and trait anxiety could predict the size of differences between the diary and the questionnaire.

Headache intensity and headache duration were, compared to the diary, overestimated on the questionnaire. At group level, median headache frequency as measured by the diary and the questionnaire was equal. Regarding headache frequency and headache intensity, age and headache severity were statistically related to errors in recall. For headache frequency, depression was also predictive of the size of recall error.

Recall errors occur when children are asked to report their headaches on a retrospective questionnaire. As compared to a prospective diary pain complaints are evaluated more negatively on a questionnaire. Other factors like age, depression and headache severity influence the way children and adolescents recall their headaches. To minimize biases it is advised to use a diary when studying recurrent headache complaints in children. 


\section{INTRODUCTION}

The epidemiology of headache and pain in children and adolescents has been frequently studied, but results vary as a result of different research designs, definitions of pain and measurement instruments used. Prevalence rates for weekly headache vary from 5.9 to $22 \%$ in children 7 to 18 years of age, whereas life-time prevalence rates from 37 to $93 \%$ have been reported. ${ }^{1}$ According to Passchier \& Orlebeke $24 \%$ of children 10 to 17 years of age are bothered by headaches once a month or more often.

Assessing pain in children is complex because few quantifiable, valid and reliable measurement instruments exist. ${ }^{3-6}$ In adults, self-report measures are currently considered as the most reliable indicators of pain. The use of self report, although the most reliable method for collecting extensive information on children's experiences with pain, is hampered by the subjective nature of pain. ${ }^{7}$ In most pediatric headache studies, self-report measures have been used retrospectively in that children are asked to recall their headache complaints over the past month or year. ${ }^{2}$ 8-10 This requires the ability to retrieve information from the long-term memory. Children, however, may not be able to remember the different dimensions of headache and may have a different perception of time due to limited cognitive abilities, ${ }^{1,5,10-12}$ Currently, little is known about the extent to which children and adolescents are able to remember the frequency and duration of painful experiences, and almost nothing is known about the ability to remember other components of pain such as intensity, quality and behavioral activities. ${ }^{13,14}$

Recall errors occur in all cross-sectional studies, that rely on respondent's selfreport. ${ }^{15,16}$ Factors related to recall errors are interference (i.e. as an individual experiences an increasing number of events, the probability of recalling any one of those events specifically declines), the length of time between the occurrence of an event and the recall of that event, the salience of the event and the respondent's psychological state, e.g. his or her mood. Dependent on their mutual coherence, these factors can lead to an over-or underestimation of the event." Other factors related to recall errors may be personal variables (e.g. gender, age, social-economic status), social desirability, interviewing technique and the mativation of the respondent. ${ }^{17}$

When recall errors are distributed non-randomly, it is called recall bias. Recall bias is a threat to the validity of all studies comparing groups, as unknown variables (e.g. age, depression) are related to recall errors, thus leading to difficulties in the interpretation of the results of such subgroup analyses. 
In adult patients with acute or chronic pain, memory for pain seems to be accurate if it refers to an acute event and is recalled after a short time interval, while it is less accurate if it refers to a chronic event and is recalled after a long interval. ${ }^{14}$ Chronic pain patients remember having experienced significantly more pain than they reported at the time the pain was actually occurring. ${ }^{18,19}$ In a study of Linton ${ }^{20}$ chronic pain patients overestimated their pain intensity after 18 months with $70 \%$. Eich et al. ${ }^{21}$ showed that in patients with chronic migraine a high current pain intensity leads to an overestimation of prior experienced pain, whereas in patients with a low present pain intensity the prior pain was rated as less severe than their hourly diaries indicated. In contrast, Jamison et al. ${ }^{19}$ and Linton ${ }^{20}$ found no relationship between current pain intensity and the remembrance of prior pain experiences. In the study of Jamison et al. ${ }^{19}$, patients who reported emotional distress, who had conflicts at home, who scored higher on the somatization scale, who were less active or who relied on medication appeared to be most inaccurate in remembering prior pain. These patients overestimated previously experienced pain intensity. Also, females tended to overestimate pain intensity more than males. Conflicting results have been reparted by Linton ${ }^{20}$. No relation was found between recall accuracy in chronic back pain patients and current depression, daily functioning or quality of sleep. According to Mogg \& Matthews ${ }^{22}$ and Bradley et al. ${ }^{23}$ state and trait aspects of depression and anxiety interact with recall error. Persons with trait and state anxiety mostly recall negative material, but persons with trait anxiety and without state anxiety recall mostly positive material. They conclude the same for the relation between state and trait depression. In a situation without state anxiety or state depression a compensating mechanism might occur that suppresses negative memories. Wright \& Morley ${ }^{24}$ and Edwards et al. ${ }^{25}$ suggest that the relationship between memory for pain and current coping is an important question.

Lander et al. ${ }^{26}$ performed a study of acute pain in children 5 to 17 years of age. Two months after the pain experience occurred, the recall of affective pain components was good. Only one study has been performed on the accuracy of recalling recurrent headache complaints in children. Andrasik et al. ${ }^{27}$ studied headache complaints in children 8 to 16 years of age. Children and their parents estimated nearly identical values for mean headache frequency, intensity and duration on a questionnaire. Headache complaints, as measured by a questionnaire, were overestimated with 56 to $112 \%$ by children and their parents, as compared to a child's diary. These differences couldn't be explained by differences in age or gender of the children. In this study other factors possibly related to recall errors were not investigated. 
In conclusion, studies on recall errors and recall bias in adult patients with acute or chronic pain show conflicting results. In children, studies on memory for pain are very limited. In addition, there is a lack of studies on memory for pain in patients suffering from recurrent pain such as headache.

Therefore this study aims firstly at investigating whether children and adolescents can recall prior headache complaints accurately. Secondly, this study aims at explaring whether the occurrence of recall errors can be explained by factors such as age, gender, headache severity, preferred pain coping strategies, trait anxiety, somatization and depression.

\section{METHODS}

\section{Design}

To investigate the accuracy of recalling headache frequency, headache duration and headache intensity among children 9 to 16 years of age with recurrent headache complaints, data on a retrospective headache questionnaire and a prospective 4-week headache diary were compared. To determine the occurrence of recall bias, age, gender, headache severity, preferred pain coping strategies, depression, somatization and trait anxiety were related to questionnaire and diary differences in headache frequency, duration and intensity.

\section{Instruments}

Headache questionnaire. - The retrospective headache questionnaire included the Waters' Headache Questionnaire $(W H Q)^{2,28}$, the Abu-Saad Pediatric Pain Assessment Tool (PPAT) ${ }^{29}$ and the Pain Coping Questionnaire (PCQ) of Reid et al. $^{31}$ Headache frequency and duration were measured by the WHQ on 7-point Likert scales. Reasonable test-retest reliability of these scales was found in post-puberty girls. ${ }^{2}$ Headache intensity was assessed by means of the $100 \mathrm{~mm}$. Visual Analogue Scale (VAS) of the PPAT. Criterion-related and construct validity of the VAS were demonstrated by Huijer Abu-Saad. ${ }^{29.30}$ A test-retest study from our group among 255 children 9 to 16 years of age revealed Spearman rank correlations for headache frequency, duration and intensity of $0.74,0.80$ and 0.54 respectively. A Dutch transiation of the PCQ was used to assess preferred pain coping strategies. The preferred coping strategies, as described by Reid et al. ${ }^{31}$ are approach, distraction and emotion-focused 
avoidance. These coping styles correspond well to related concepts as emotional distress, pain controllability and functional disability.

Moderate to high internal consistencies were found for all subscales underlying the three higher-order coping styles.

Headache diary. - In a prospective structured 4-week headache diary, modeled after Richardson et $a .^{32}$ and Langeveld et $a ._{.}{ }^{33}$, headache frequency, duration, intensity and severity were recorded four times a day; at breakfast, lunch, dinner and bedtime. The first question of the headache diary measures the presence and experienced severity of a headache complaint on a 6-point Likert scale. If a headache complaint occurred, the time the headache began and stopped was recorded, and experienced headache intensity was reported on a $100 \mathrm{~mm}$. VAS. Several authors have shown a prospective headache diary to be valid for children over 7 years of age, when comparing children's headache reports with parent's reports. ${ }^{12,27,32}$ The comprehensibility of the diary was tested in a pilot study and proved to be satisfactory.

Other questionnaires. - A shortened version of a Dutch Depression questionnaire for children (KDVK) was used to assess depression. ${ }^{34}$. The KDVK is a screening instrument and consists of 9 statements that children can agree with or not. $A$ score of 4 or more is an indication for depression. The KDVK correlates highly with similar concepts like neuroticism and social inadequacy. According to $\mathrm{Wit}^{34}$ percentages false-positives and false-negatives are low, 3.7\% and $4.8 \%$ respectively. A Dutch translation of the State-Trait Anxiety Inventory for Children of Spielberger et al. ${ }^{35}$, the Self-assessment questionnaire in children $(\mathrm{ZBV}-\mathrm{K})^{36}$ measures state and trait anxiety. In this questionnaire state anxiety refers to the moment the headache occurred. In the literature no indications were found for a relation between this kind of state anxiety and recall errors. Therefore, in this study state anxiety was not selected as a variable. Trait anxiety refers to feelings of anxiety" a child usually has and was measured by 20 statements, scored on 3-point Likert scales. For the ZBV-K high internal consistencies and stability coefficients were found, and construct and discriminant validity was shown by Bakker et al ${ }^{36}$ Somatization was assessed by means of the Children Somatization Inventory (CSI). The CSI is a 35-item questionnaire measuring the intensity of self-reported somatic complaints on 5point Likert scales. Reliability and validity of the CSI are satisfactory. ${ }^{37,38}$ Table 5.1 presents an overview of all variables and measurement instruments used in this study. 
Table 5.1 Study variables and measurement instruments

\begin{tabular}{|c|c|c|}
\hline Variable & Instrument & Measurement scale \\
\hline \multirow[t]{2}{*}{$\begin{array}{l}\text { Headache } \\
\text { frequency }\end{array}$} & $\begin{array}{l}\text { Waters' Headache } \\
\text { Questionnaire } 2,28\end{array}$ & $\begin{array}{l}\text { 6-point Likert scale with an open answering possibility. } \\
\text { (1=about once a year: } 2=\text { several times a year", about... } \\
3=a b o u t \text { once a month: } 4=\text { several times a month. } \\
\text { about... } 5 \text { =about once a week: } 6=\text { several times a week. } \\
\text { about..) }\end{array}$ \\
\hline & Headache diary $y^{32,33}$ & Count of the number of headache attacks recorded. \\
\hline \multirow[t]{2}{*}{$\begin{array}{l}\text { Headache } \\
\text { duration }\end{array}$} & $\begin{array}{l}\text { Waters' Headache } \\
\text { Questionnaire }\end{array}$ & $\begin{array}{l}\text { 7-point Likert scalle. } \\
\text { ( } 1=\text { less than half an hour: } 2=\text { between half an hour and } \\
\text { an hour: } 3=\text { between an hour and half a day: } 4=\text { between } \\
\text { half a day and a day; } 5=\text { one to two days; } 6=\text { between } \\
\text { two days and a week: } 7=a \text { week or longer) }\end{array}$ \\
\hline & Headache diary ${ }^{32,33}$ & Median number of minutes that headache attacks last. \\
\hline \multirow[t]{2}{*}{$\begin{array}{l}\text { Headache } \\
\text { intensity }\end{array}$} & $\begin{array}{l}\text { Pediatric Pain } \\
\text { Assessment Tool }\end{array}$ & $\begin{array}{l}100 \mathrm{~mm} \text {. Visual Analogue Scale; anchors 'I have no pain' } \\
\text { and 'I have very severe pain'. }\end{array}$ \\
\hline & Headache diary $y^{32,33}$ & Median score of $100 \mathrm{~mm}$. Visual Analogue Scales. \\
\hline $\begin{array}{l}\text { Headache } \\
\text { severity }\end{array}$ & Headache diary ${ }^{32,33}$ & $\begin{array}{l}\text { Median score of 6-point Likert scales. } \\
\text { ( } O=\text { =no headache; } 1=\text { headache-I am only aware of it if I } \\
\text { pay attention to it: } 2=\text { headache-but I can ignare it at } \\
\text { times: } 3=h e a d a c h e-I \text { can't ignore it but I can do my } \\
\text { usual activities; } 4=h e a d a c h e-i t ' s \text { difficult for me to } \\
\text { concentrate; } 5=h e a d a c h e-s u c h \text { that I can't do } \\
\text { anything) }\end{array}$ \\
\hline
\end{tabular}

Preferred

coping

strategies

Questionnaire $^{31}$

Depression

Trait anxiety

Somatization
Self-assessment Questionnaire for Children $^{35,36}$

Depression Questionnaire for Children $^{34}$

Children Somatization Inventory ${ }^{37,38}$
39 items, encompassing three higher-order factors, measured on 5-point Likert scales indicating how often each of 39 strategies was used (1=never:" $2=$ hardly ever; 3 =sometimes, 4=often: 5=very often). For each higher-order factor, a mean score was calculated.

9 statements with which children can agree or not, coded as 0 or 1. A sum-score was calculated and children were divided in 2 groups: (indication of) depression (sum-score $\geq 4$ ) or no (indication of) depression (sum-score 4 ).

20 statements scored on a 3-point Likert scale (1=hardly ever; 2 =sometimes; 3 =orten), of which a sum score was calculated

35 items on a 5-point Likert scale ( $t=$ mo problems at all: 2=some problems: 3=problems; 4=quite some problems: 5 =nany problems) of which a sum scare was calculated. 


\section{Procedures}

First, as part of a larger cross-sectional study the headache questionnaire was filled in by 2871 children 9 to 16 years of age in the classroom setting of their elementary or high school. The children were given oral instructions by the researcher on how to complete the headache questionnaire, illustrated by several examples. Approximately 3 months later children who experienced headache complaints at least 4 times a month, as indicated on the questionnaire, were invited to monitor their headaches daily in a prospective diary for four weeks. In a group meeting, children were instructed on the use of the diary. During this meeting, the KDVK, ZBV-K and CSI were also administered.

\section{Analyses}

Subjects. - Children who did not return all four weeks of the diary or who missed more than $10 \%$ of the valid items of the questionnaires were excluded from analysis. For subjects with less than $10 \%$ missing items, missing values on somatization, trait anxiety and coping were substituted by the mean of the subject's non-missing items under the same sub-scale. If the number of missing items exceeded $25 \%$ of the number of sub-scale items, the sub-scale was ascribed as a missing value. Drop-outs (i.e. children who did not return the complete diary and who missed more than $10 \%$ of the questionnaire items) were, if possible, compared to subjects who were not excluded on all study variables using T-tests, non-parametric tests and Chi-square tests.

Recall error. - Headache frequency, as measured with the WHQ, was converted into the number of headache attacks per 4 weeks to be able to compare the questionnaire score to the number of headache attacks as reported in the diary. For each child, median headache intensity, as scored at the diary, was calculated to compare with the intensity score of the PPAT. Headache duration at the diary, measured in minutes per headache attack, was transformed to the 7 -point Likert scale measuring headache duration of the questionnaire. Subsequently, median headache duration was calculated for each child. Because of a non-normal distribution of the data. Wilcoxon tests were used to compare questionnaire scores of headache frequency, duration and intensity to diary scores. Furthermore, Spearman rank correlations were calculated for all variables.

Recall bias. - Five dependent variables were constructed indicating the concordance between diary and questionnaire data. For headache frequency and headache intensity, a differential (diary minus questionnaire) as well as a 
proportional (diary divided by questionnaire) score was constructed for each child. For headache duration, a dichotomous agreement rate was calculated by comparing the values on questionnaire and diary and attributing a score of 1 ('yes') if these values were equal and a score of $O$ ('no') if not.

Sum scores were calculated for the independent variables trait anxiety and somatization. For each coping strategy, a mean score was derived of the underlying items. As for headache severity, the median value of the reported diary scores was used for each child. Depression was dichotomized as 'yes', (indication of) depression, or 'no', no (indication of) depression. Multiple linear and logistic hierarchical backward regression analyses were performed to study whether age, gender, headache severity, trait anxiety, depression, somatization and preferred coping strategies could predict the size of recall error.

\section{RESULTS}

\section{Subjects}

The number of children starting with the headache diary was 212. Of these children, $186(88 \%)$ completed all four weeks of the diary. Five children were lost because of more than $10 \%$ missing values on the questionnaires. As a result, $181(85 \%)$ remained for analysis. For all research variables assessed by the questionnaires, the group of dropouts has been compared to the group of children who completed the diary. No statistically significant differences between the dropout group and the other respondents were found concerning age, gender, headache frequency, duration, intensity, trait anxiety, depression, somatization and preferred coping strategies. The mean age of the remaining 181 children was 11.5 years (SD 2.1). Of these children $59(32 \%)$ are boys. One third of the sample attends high school. According to the questionnaire, all children experienced headaches frequently, $30 \%$ experiencing headaches several times a month and $70 \%$ experiencing headaches once a week or more often.

Table 5.2 Comparison of headache parameters in questionnaire and diary (per 4 weelks)

\begin{tabular}{lcccc} 
& Questionnaire & Diary & P-value $^{\mathrm{a}}$ & Correllation \\
\hline Median frequency & 9 & 9 & 0.24 & 0.16 \\
Median intensity & 65 & 37 & 80.01 & 0.21 \\
Median duration & 4 & 3 & $<0.01$ & 0.21 \\
\hline
\end{tabular}

Wilcoxon tests have been used to compare questionnaire and diary scores 


\section{Recall error}

As shown in table 5.2, both on the questionnaire and the diary, the median number of headache complaints per 4 weeks was $9(p=0.24)$.

During the diary period, 4 children ( $2 \%$ ) did not have a headache experience at all. According to the diary $71 \%$ of the children experienced headaches once a week or more often and $27 \%$ had headaches several times a month. Although median headache frequency of the questionnaire and the diary was equal $(p=0.24)$, a low Spearman's rank correlation of 0.16 was found, indicating that children show high intra-individual variance in the way they answered the questionnaire and the diary. Fifty-three children (29\%) remained relatively stable in their estimation of headache frequency (overestimation or underestimation of maximally 3 headache complaints per 4 weeks). An overestimation of more than 3 headache complaints on the questionnaire was found in $40 \%$ of the children. The remaining $31 \%$ underestimated their headache frequency with more than 3 headache complaints per 4 weeks.

Headache intensity scores at the diary were much lower than at the questionnaire. Median headache intensity according to the diary was 37 , whereas median headache intensity based on the questionnaire was 65 ( $p<0.01)$. Spearman's rank correlation of questionnaire and diary was 0.21 . Children appear to strongly overestimate their headache intensity (with about $71 \%$ ) when answering the headache questionnaire.

Headache duration was also significantly overestimated at the questionnaire (see table 5.3). Median headache duration was 3 (lasting between an hour and half a day) according to the diary and 4 (headache lasts between half a day and a day) according to the questionnaire ( $p<0.01$ ).

Table 5.3 Percentages of children scoring in each category of headache duration according to the questionnaire and the diary.

Duration categories Questionnaire (\%) Diary $(\%)$

$1=$ less than half an hour 3.3 8.5

$2=$ between half an hour and an hour 14.4

$3=$ between an hour and half $a$ day

19.3

54.2

4 = between half $a$ day and $a$ day

27.1

$5=$ one to two days 25.4

$6=$ two days to a week

$7=a$ week or langer 
The percentages of children scoring in each category according to the questionnaire and the diary are shown in table 3. Most children (46\%) had a difference of one duration category when comparing the questionnaire and the diary. Eighteen percent differed 2 categories, $11 \% 3$ categories, $5 \%, 4$ categories and $1 \% 5$ categories. Of all children $20 \%$ chose exactly the same category.

\section{Recall bias}

The mean sum scores of trait anxiety and somatization were 36 (SD 7) and 28 (SD 16) respectively. According to the criteria of the KDVK $40 \%$ of the children were indicated as depressed. Children were most inclined to use the coping strategy distraction (mean 2.3, SD 0.8). The mean scores for the coping styles emotion-focused avoidance and approach were 2.0 (SD 0.7) and 2.1 (SD 0.5) respectively. Median headache severity was 2.5 according to the diary.

In table 5.4 the results of the regression analyses are shown for all headache parameters. These analyses aimed at investigating recall bias in an explorative way. Therefore, a $p$-value less than 0.10 was considered as indicative of recall bias.

Table 5.4 Results of the regression analyses for recall bias.

\begin{tabular}{lllll} 
Variables & $\begin{array}{l}\mathrm{P} \text {-value } \\
\text { overall F-test }\end{array}$ & $\begin{array}{l}\beta^{-} \\
\text {value }\end{array}$ & Predictors & $\begin{array}{l}\beta- \\
\text { value }\end{array}$ \\
& & & value \\
\hline
\end{tabular}

Headache frequency:

differential score ${ }^{\mathrm{a}}$

Headache frequency:

proportional score

Headache intensity:

differential score ${ }^{a}$

Headache intensity:

proportional score
N.S. $(0.43)$

0.04

0.85

Age

$0.1 \quad 0.05$

Depression

Headache

$0.36<0.01$

severity

N.S. $(0.20)$

0.08

0.87

Age

$-0.03 \quad 0.07$

Headache severity

Headache duration

N.S. $(0.18)$

a differential score was calculated by substracting the questionmaire score from the diary score.

A proportional score was calculated by dividing the diary score by the questionnaire score.

' For each child the questionnaire score was compared to the diary score and scored as 'yes' or 'no' agreement. 
For the differential scores of headache frequency and headache intensity, none of the independent variables seemed to contribute to explaining the size of differences between the diary and the questionnaire $(p=0.43$ and $p=0.20$ respectively). Also, none of the variables explained the variance of the agreement rate of headache duration $(p=0.18)$.

However, for proportional headache frequency three variables appeared to predict the size of recall error, namely age $(p=0.05)$, depression $(p<0.01)$ and headache severity $(p=0.08)$. Dependent on their mutual coherence and the values of these variables, this may result in an over- or underestimation of headache frequency as measured by the questionnaire. Older children are inclined to underestimate their headache frequency more than younger children do when answering a questionnaire. The younger the children are the less they underestimate their headache frequency retrospectively. A higher experienced headache severity is associated with an overestimation of the number of headache complaints in the questionnaire. Depressive children underestimate the number of headache complaints on a questionnaire more than their nondepressive counterparts.

Age and headache severity were also significantly related to proportional headache intensity ( $p=0.07$ and $p<0.01$ respectively). The effects of age and headache severity on the recall of headache intensity, however, vary from the effect on recall of headache frequency. Older children overestimate their headache intensity on the questionnaire more than younger children do and a higher headache severity is associated with less overestimation of headache intensity on the questionnaire.

\section{CONCLUSIONS AND DISCUSSION}

This study firstly aimed at investigating whether children 9 to 16 years of age can recall prior headache complaints accurately. Secondly, the predictive value of other factors possibly related to recall errors was investigated.

Headache intensity and headache duration were strongly overestimated according to the questionnaire as compared to the diary. At group level, headache frequency scores appear to be equal, assessed either retrospectively or prospectively. However, correlations are low for all headache parameters, indicating that intra-individual variance exists in the way children answer the diary or the questionnaire. These results are similar to the results found in other studies on the memory of recurrent or chronic pain. ${ }^{19-21,27}$ Retrospectively. pain complaints are evaluated more negatively as compared to a prospective 
diary. This may in part explain the wide variation found in the prevalence and incidence rates of headache complaints $s^{1,2}$, as many different measurement instruments with different time frames have been used in previous studies.

To investigate the existence of recall bias, the construction of the dependent variable as a proportional measure appears to be more meaningful than a differential calculation. A proportional measure takes into account the notion that a difference of $10 \mathrm{~mm}$ in pain intensity on the VAS is more indicative of recall bias for a child with an initial score of 50 as for a child with an initial score of 80 . In other studies investigating recall bias, recall errors were calculated either differential or proportional. ${ }^{18-21}$ This may partly explain the conflicting results found in these studies. As was shown in table 4, the choice for a differential or proportional measure also affected our results on recall bias.

Regarding headache duration, no evidence was found for the existence of recall bias. Because of the high aggregation level however, the agreement rate of headache duration may not have been sensitive enough to detect differences in accuracy of recall. Headache duration, measured in minutes in the diary was first categorized to compare with the questionnaire score. Second, the questionnaire score was compared to the diary score and agreement was recorded as 'yes' or 'no'. In this process loss of information may have occurred. For both headache frequency and intensity, age and headache severity were found to be predictive of the size of recall error. Remarkably, older children appear to be less accurate in recalling headache frequency and intensity than younger children. In the study of Andrasik et al. ${ }^{27}$ age was not predictive of differences in recall accuracy. These findings suggest that younger children are not less able than older children to recollect information from the long-term memory, as has been stated by other authors. $1,5,10-12$

Headache severity is associated with an overestimation of headache frequency on the questionnaire, but with more accurate reports of headache intensity. Possibly, children with a higher headache severity experience their headaches as interfering with their daily activities and are therefore inclined to overestimate the number of headaches. These children may at the same time pay more attention to their headaches, resulting in a more accurate estimation of headache intensity. However, to our knowledge the influence of limitations in daily activities on recall error has not been addressed in the literature and further research is thus needed to confirm this hypothesis.

The results on the predictive value of depression are inconclusive. Depressed children seem to underestimate their headache frequency more than nondepressed children and adolescents. For headache intensity depression was not a 
predictor of recall error. In adults, depression was related to recall errors according to several authors. ${ }^{22.23}$ In our study, depression was measured by the KDVK. This questionnaire is used only as a screening instrument for depression. In future, a more comprehensive assessment of depression is required to study the effect of depression on recall bias. In addition, the number of children in our study with a high score on the depression questionnaire was small. A focused study on recall in depressed children may give more information.

Gender, somatization, trait anxiety and preferred coping strategies were not related to questionnaire and diary differences in this study.

Our results underpin the importance of distinguishing qualitative and quantitative aspects of pain. Most studies on pain recall in adults have focused on pain intensity. ${ }^{18-21}$ In children, only Andrasik et al. ${ }^{27}$ studied the recall of other parameters as headache frequency and duration. In their study, age and gender were not related to errors in recall. However, we found indications that age and headache severity are predictive of recall errors. Older children show less accuracy in their reports of priar pain than younger children. This may result in an underestimation of headache frequency and an overestimation of previously experienced pain intensity measured by a questionnaire in epidemiological studies among adolescents. Moreover, the validity of intervention studies is threatened, when the effect of an intervention is calculated as the difference between pre- and post-test questionnaires. For instance, age can bias the results of an intervention study on headache intensity and the intervention may be regarded as more effective in the older age group simply because older children overestimate their headache intensity more than younger children. To minimize recall bias in studies using questionnaires, it is advised to use proportional effect measures.

The importance of recognizing recall error and recall bias is eminent. However, one could argue that a headache questionnaire does reflect a subjective judgement of the previously experienced pain. Moreover, questionnaires are often more feasible, less costly and less time-intensive than diaries. Presumably, the size of recall error also depends on the time frame used in the questionnaire, with shorter time frames being less sensitive to recall errors. The choice between a diary and a questionnaire should be based on the purpose of the study. Imprecision in large cross-sectional studies may be less severe than in intervention studies. One should be aware, however, of the possible bias when using questionnaires with longer time frames.

There are some limitations to our study. Firstly, the children who were asked to fill in the diary were selected based on the answers they had given on the questionnaire. Children who experienced frequent and serious headaches were 
invited to complete the diary. It may be that for some of these children their headaches reduced during the period between the questionnaire and the diary. because they were selected just after a 'bad' headache period.

Secondly, causal relations cannot be established using a cross-sectional design. The results of the regression analysis must be interpreted carefully and causal inferences drawn with caution. More powerful research designs should be used to establish provisional relationships, e.g. selection of groups of younger and older children of whom questionnaire and diary data will be compared. Also, variables may interact and other variables might be related to accuracy of recall such as experienced pain intensity and psychological state at the moment the questionnaire is administered. Studies investigating the mechanisms of pain recall could provide insight in the factors important for accurate pain memory.

In conclusion, time seems to change the way children remember their headaches and the different dimensions of headache. Age and headache severity seem to influence the size of recall errors. To minimize biases it is advised to use a diary when studying recurrent headache complaints in children.

\section{REFERENCES}

1. Olesen J, Tfelt-Hansen P, Welch KMA. The headaches. New York: Raven Press, 1993.

2. Passchier J, Orlebeke JF. Headaches and stress in schoolchildren: an epidemiolagical study. Cephalalgia 1985:5:167-76.

3. Ferrari P. Incorpora G, Cocuzza M, Guerra L, Lester A, Soriani S, Pinca A, Scanabissi E, Migliore MR, Tavoni E, Tozzi E, Zammatara Bogliolo $C$, d'Ancona ML. Multicenter study of childhood headache. Children's Nerwaus System 1994;10:455-457.

4. Goodman JE, MCGrath PJ. The epidemiology of pain in children and adalescents: a review. Pain 1991:46:247-264.

5. MCGuire DB. Measuring pain. In: Frank-Stromberg $M$, ed. Instruments for clinical nursing research. Norwalk, Appleton \& Lange, 1989, p. 333-356.

6. Rothner AD. Headaches in children and adolescents. The Clinical Journal of Pain 1989:5:6775.

7. McCaffery M. Nursing management of the patient with pain. Philadelphia: JB Lippincott, 1979.

8. Brattberg $G$. The incidence of back pain and headache among Swedish school children. Quality of Life Research 1994:3:527-531.

9. Abu-Arafeh I, Russell G. Prevalence of headache and migraine in school children. $B M J$ 1994:309:765-769.

10. Mortimer MJ, Kay J, Gawkrodger DJ, Jaron A, Barker DC. The prevalence of headache and migraine in atopic children: an epidemiological study in general practice. Headache $1983: 427-431$.

11. Biemer PP, Groves RM, Lyberg LE, Mathiowetz NA, Sudman 5. Measurement errors in surveys. New York: John Wiley \& Sons, 1991 
12. Labbe EE, Williamson DA, Southard DR. Reliability and vallidity of children's reports of migraine headache symptoms. Journal of Psychopathology and Behavioral Assessment 1985:7(4):375-83.

13. Erskine A, Marley S, Pearce S. Memory for pain: a review. Pain 1991;41:255-265.

14. Fienberg SE, Loftus EF, Tanus JM. Recalling pain and other symptoms. Mribank Memorial Fund Quarterly 1985;63:583-597.

15. Lippman A, Mackenzie S6. What is recall bias and does it exist? Progressive Clinical Biological Research 1985;163c:205-9.

16. Raphael KG, Cloitre M. Does maod-congruence or causal search govern recall-bias? A test of life event recall. Journal of Clinical Epidemiology 1994:47:555-64.

17. Coughlin SS. Recall bias in epidemiological studies. Journal of Clinical Epidemialogy 1989:43:87-91.

18. Linton $S J$, Melin L. The accuracy of remembering chronic pain. Pain 1982:13:281-285.

19. Jamison RN, Sbrocco T, Parris WCV. The influence of physical and psychosocial factors on accuracy of memory for pain in chronic pain patients. Pain 1989:37:289-294.

20. Linton 5J. Memory for chronic pain intensity: correlates of accuracy. Perceptual and Motor Skills 1991:72:1091-1095.

21. Eich E, Reeves JL, Jaeger B, Graff-Radford SB. Memory for pain: relation between past and present pain intensity. Pain 1985:23:375-379.

22. Mogg K, Mathews $A$. Is there a self-referent mood-congruent recall-bilas in anxiety? Behaviour Research and Therapy 1990;28:91-92.

23. Bradley B, Magg K, Galbraith M. Perrett A. Negative recall-bias and neuroticism: state vs trait effects. Behaviour Research and Therapy 1993:31:125-127.

24. Wright J, Morley S. Autobiographical memory and chronic pain. British Journal of Clinical Psychology 1995;34:255-265.

25. Edwards L. Pearce S, Collett B, Pugh R. Selective memory for sensory and affective information in chronic pain and depression. British Journal of Clinical Psychology 1992;31:239-248.

26. Lander J, Hadgins M, Fowler-Kerry S. Children's pain predictions and memories. Behaviaur Research and Theropy 1992:30:117-124.

27. Andrasik F, Burke EJ, Attanasio V, Rosenblum EL. Child, parent and physician reports of a child's headache pain: Relationships prior to and following treatment. Headache 1985;25 $(11): 421-25$.

28. Waters WE. The epidemiology of migraine. Bracknell-Berkshire: Boehringer Ingelheim, 1974.

29. Huijer Abu-Saad H, Kroonen E. Halfens R. On the development of a multidimensional Dutch pein assessment tool for children. Poin 1990;43:249-256.

30. Abu-Saad H. Assessing children"s responses to pain. Pain 1984;19:163-171.

31. Reid GJ, Gilbert CA, McGrath PJ. The Pain Coping Questionnaire: preliminary validation. Pain 1998:76:83-96.

32. Richardson GM, MCGrath PJ, Cunningham SJ, Humphreys PH. Validity of the headache diary for children. Headache 1983:23:184-187.

33. Langeveld $J H_{1}$ Koot HM, Passchier J. Headache intensity and quality of life in adolescents. How are changes in headache intensity in adolescents related to changes in experienced quality of life. Headache 1997;37:37-42.

34. Wit CAM de. Depression questionmaire for children, DVK and KOVK, Manual (in Dutch). Amersfoort: Acco, 1987. 
35. Spielberger CD. Manual for the State-Trait Anxiety Inventory for children. Palo Alto, CA: Consulting Psychologists Press, 1973.

36. Bakker FC, Wieringen PCW wan, Ploeg HM van der, Spielberger $C D$. Manual for the selfassessment questionnaire in children (in Dutch). Lisse: Zwets \& Zeitlinger, 1989.

37. Walker LS, Greene JW. Children with recurrent abdominal pain and their parents: more somatic complaints, anxiety and depressian than ather patient families? Journal of Pediatric Psychology 1989:14:231-243.

38. Gorber J. Walker LS, Zeman J. Somatization symptoms in a community sample of children and adolescents: further validation of the Children's Somatization Inventory. A Journal of Consulting and Clinical Psychology 1991:3:588-595. 


\section{Chapter 6}

Effects of a self-help program on headache in children

Bandell-Hoekstra I, Huijer Abu-Saad H, Passchier J, Frederiks C, Feron F, Knipschild P. Effects of a self-help program on headache in children. Submitted. 


\section{ABSTRACT}

The prevalence of recurrent headaches in the general population of children and adolescents is high. The "Help Yourself cope with stress and migraine" training has been successful in adolescent migraine patients referred to a headache clinic. Aim of our study was to expand the target population to 9 to 17 year olds with frequent headaches, in a preventive Youth Health Care setting in the Netherlands. Subjects $(n=158)$ have been randomly assigned to the experimental Help Yourself group or the placebo-control group. We compared headache frequency, intensity, duration and Total Headache Index (THI) at pre-test, with post-test and 3, 6 and 12 months follow-up assessments and found no differences between both groups. Headache frequency reduced from 11 to 3 headaches in four weeks. 


\section{INTRODUCTION}

Children and adolescents report recurrent, primary headaches quite commonly. An overview of cross-sectional studies in the general population shows a prevalence of headaches every week ranging between $6 \%$ and $29 \%{ }^{1}$ Prospective studies on headache persistence later in life are rare, but Bille has shown that $60 \%$ of children with migraine still, or again, experience migraine after the age of 30 years. ${ }^{2}$

The role of psychological factors in primary headaches is ubiquitous. Psychological stress is reported to be the most frequent precipitating factor for headache, both in adults ${ }^{3-5}$ and in children. ${ }^{6-9}$ Recent studies have shown physiological evidence for the role of psychological stress as a precipitant of migraine. ${ }^{10}$ The role of stress in tension-type headache needs further investigation, but it is generally acknowledged that stress can cause muscle tension, which may lead to headache. According to Pennebaker's symptom report theory, stress may also lead to a higher probability of pain perception. ${ }^{3}$ In fact. stress appears not only to precipitate but also to exacerbate or maintain headache activity. ${ }^{11}$

Mal-adaptive attitudes, appraisals, expectations and behaviors contribute to stress and are thus assumed to increase the risk of headache. ${ }^{12,13}$ Cognitivebehavioral training is focused on these stress-inducing mal-adaptations and is aimed at teaching coping strategies. Relaxation techniques are a specific set of coping strategies that can relieve muscle tension, reduce stress, increase the sense of mastery and divert attention from the pain. ${ }^{14,15}$

Psychological treatments are often preferred in children; there is reluctance to use (prophylactic) medication. ${ }^{16,17}$ Moreover, psychological training early in life may facilitate the use of these strategies later on.

10,18,19 Self-help programs appear to be equally effective, compared to therapist assisted programs in children. ${ }^{17}$ They require greater involvement in and responsibility for the patients' own treatment, which facilitates increased self-efficacy and cognitive coping. 20 Self-efficacy, or perceived capacity to exercise self-control over cognitive, behavioral, and affective responses to stressful events, has been shown to buffer the impact of stress on headache frequency. ${ }^{21}$ Self-help programs are less costly, less threatening, and more easily accessible, thus suitable for a large number of potential consumers. 22

The "Help Yourself cope with stress and headache" training ${ }^{23}$ has been developed for adolescents with migraine and has shown to be successful in 11 to 18 year old children who had been referred to a migraine clinic. ${ }^{17}$ 
The prevalence of frequent primary headaches in the general population of children and adolescents emphasizes the need for preventive measures. Enhancing self-management skills and preventing headache chronicity have become major goals. Hence, the purpose of our study was to test the "Help Yourself" training in a preventive Youth Health Care setting in the Netherlands. We have expanded the target population of the Help Yourself program to 9 to 17 year old children in the general population, who experience frequent primary headaches.

\section{METHODS}

\section{Subjects}

Subjects come from a general population of 2,871 school children in grades 6,7 and 8 of elementary schools (aged 9-12 years) and grades 1 to 4 of high schools (aged 12-17 years) in the city and region of Maastricht. The Netherlands. These children have participated in a cross-sectional study, ${ }^{1}$ in which they have filled out Waters' Headache Questionnaire (WHQ) ${ }^{4,24,29}$ and the Pediatric Pain Assessment Tool (PPAT). 26,27 Independently, two raters scored each questionnaire in terms of possible inclusion. Only if both raters agreed, children were invited for the intervention study. 433 selected children received an information letter. It explained the two-groups design of the study, the randomization as well as the measurements and training procedures. Then, all selected children were phoned for their reply and 250 children were included in the randomization procedure.

\section{Procedure}

The Medical Ethics Committee of the Academic Hospital and University of Maastricht. The Netherlands, approved of the study. The study was designed as a Randomized Clinical Trial, involving an experimental group and a control group. with a pre- and posttest and 3,6 and 12 months follow-up.

After the child's and parental oral consent, children were randomly assigned to the experimental or the control group. Children were assigned to the same group if they attended the same elementary school, if they attended the same grade in high school, or if they were siblings, in order to prevent treatment contamination.

Then, children were invited to a headache-related medical examination at the school physician's office, in order to exclude secondary headaches. 
The experimental as well as the control group received a training program, which was identical with regard to form but different with regard to content.

The experimental group received the "Help Yourself cope with stress and headache" training program, 17,23.28 which had been translated and slightly adapted for use in the Netherlands. The program encompassed relaxation exercises, distraction techniques and cognitive skills such as positive thinking. assertiveness, and problem solving. The control group received the Learn More training program, which had been specifically designed as a credible placebo in this study. It encompassed the registration of potential headache triggers, such as food and fluid intake, rest and exercise, the weather, room temperature, and stress. Purpose of the Learn More training program was to mimic the nontherapeutic elements of the experimental training, i.e. the child's attention to his or her headache, and to provide an equal amount of attention by the researchers, the school physician, and others such as parents and peers. However, the therapeutic elements of the experimental training, i.e. cognitive and relaxation training, were not included in the Learn More training. The content of both training programs is outlined in table 6.1.

Table 6.1 Summary of content of the Help Yourself (experimental) and the Learn More (control) training pragram, described for every week. Relaxation exercises are in italic.

Help Yourself W Week

Learn More

Introduction: treatment rationale headache diary (pre-test) signs of stress, stress and headache

"Tense-release" relaxation

negative and positive thinking

"fense-release" relaxation

unrealistic ideas

"release without tension" relaxation

distraction, thought stopping

"release without tension relaxation

distraction using fantasy

relaxation with fantasy

Assertiveness

mini-relaxation

problem solving, a smile and a twinkle mini-relaxation

anticipating / dealing with headache

headache diary (post-test)
$1-4$

5

6

7

8

9

10

11

$12-15$
Intraduction: treatment rationale headache diary (pre-test)

headache types

headache trigger: food

sensory triggers

theat and close environment

rest and activity balance

stress

evaluation of triggers

conclusion

headache diary (post-test) 
Both the Help Yourself and the Learn More training program encompassed one group session, in which written informed consent was obtained from parent and child, and information on the rationale of the training and instructions on the measurement instruments were provided. Baseline measures were taken on anxiety and depression of the child.

The program consisted of four weeks of keeping a headache diary (pre-test), sever weeks of training by means of written information and home assignments, and another four weeks of keeping a headache diary (post-test). At the start of each training week, children received the chapter, exercise forms, and cassettetape by mail. At pre-test and post-test and at all follow-up measurements, participants were phoned once by one of the researchers (IB-H) or the research assistant (both health care scientists), for motivation and further instruction on keeping the diary. During the 7 weeks of training, the participants in both groups were phoned once every two weeks, for feedback and motivation. Phone calls were made based on therapist guidelines with regard to the content of the call, possible questions raised by children and answering instructions.

\section{Measures}

\section{Assessment method}

All outcome parameters were assessed with a diary that was kept by the child and was filled out four times daily. At pre-test and post-test the headache diary was kept for four weeks in a row. At 3,6 and 12 months follow-up, the headache diary was kept for two weeks and results were multiplied by 2. Headache diaries had to be returned weekly to the researchers by mail.

The headache diary was based on earlier work. ${ }^{29,30}$ First, the child had to fill in whether he or she had experienced a headache since the last report. Headache burden (score 0-5) was rated as zero in case no headache had been experienced and the diary needed no further filling out until the next reporting time. In case of a current headache episode or headache in the previous hours, the child had to rate the headache burden between 1 and 5, from "1=Headache: I am only aware of it when I pay attention to it", to " $5=$ Headache: such that I can't do anything". "17 Starting and ending time of the headache episode had to be recorded too. One headache episode lasted as long as the headache pain continued, e.g., one episode could last 10 minutes or one week. The criteria of the International Headache Society ${ }^{31}$ regarding the duration of an episode were followed. That means that if a child with a headache fell asleep, the duration of the episode included sleeping time. The child was asked to rate the peak pain 
intensity in the previous period of the episode. The headache intensity was assessed on a 0-100 $\mathrm{mm}$ Visual Analogue Scale (VAS). ${ }^{27}$ In addition to the headache diary, the children filled out a short retrospective questionnaire on headache frequency, intensity and duration in the past 4 weeks, at the end of the post-test and follow-up periods.

At baseline, trait-anxiety was assessed using the $Z B V-K,{ }^{32}$ the Dutch version of the State-Trait Anxiety Inventory for Children. ${ }^{33}$ Sum scores range between 20 and 60. In addition, a Dutch screening list with 9 dichotomous items on depression, the k-DVK was administered. ${ }^{34}$ A sum-score of 4 or higher is an indication of depression, while a sum-score of between 7 and 9 is a strong indication.

After post-test headache assessment, children filled out an ad hoc questionnaire evaluating their activities and results with regard to treatment ("process evaluation").

\section{Outcome parameters}

The following variables are the primary outcome parameters. Headache frequency was calculated as the number of headache episodes in four weeks. Headache intensity was the mean pain intensity of the episodes in four weeks. Headache duration was the sum in hours of the duration of all episodes in four weeks. Total Headache Index (THI) was the sum of headache burden in four weeks, ranging between 0 and 560 (4 times a day $\times 7$ days a week $\times 4$ weeks $\times$ burden between 0 and 5).

Secondary outcome parameters have been included as well. Clinically significant improvement of the primary outcome parameters was calculated as the percentage of children with at least a $50 \%$ decrease at post-test. Headachefree days were calculated as the sum of days without headache in four weeks. Complete absence of headache was recorded. Medication intake was the frequency of intake of pain medication in four weeks, regardless of the dose or type of medication. School absence was the four weeks sum of the number of hours missed at school as a consequence of headache and the number of doctor visits in four weeks was reported.

Variables of the process evaluation encompassed time spent weekly on the treatment program, satisfaction with the results on a 0-100 VAS, and what had been learned.

\section{Missing values}

Data were included in the statistical analyses if at least $50 \%$ of the headache diary weeks at post-test and follow-ups had been returned. Otherwise, a proxy 
score for headache frequency, intensity, and duration was obtained using the retrospective questionnaire. If the questionnaire data was unavailable too, children were regarded lost-to-follow-up for that measurement moment. Analyses were carried out following the intention-to-treat principle. Noncompliers of the training programs were defined as children who returned less than 5 weeks of the headache diary during the 7 weeks training. Non-compliers were still included in the analyses of their group when any data was available (intention-to-treat).

\section{Statistical analysis}

Descriptive statistics were used to look for differences between both groups on selection criteria and baseline characteristics after randomization, and due to loss-to-follow-up and non-compliance.

The difference between both groups was described with regard to the change in score between pre-test and post-test, and pre-test and follow-ups on the primary outcome parameters. $95 \%$ Confidence intervals of the difference between both groups were calculated. The mean scores on the primary outcome parameters at pre-test, post-test, and 3,6 and 12 months follow-up in both groups were graphically compared.

The results on the secondary outcome parameters were described. In the additional analysis, study results within the subgroup of treatment compliers were described. We also calculated the $95 \%$ confidence intervals of the difference in change (pre-test minus post-test) in THI between both intervention groups within a subgroup comparable to the sample in the study of McGrath et al. ${ }^{17}$ Differences between subgroups based on baseline variables such as gender, age, depression and anxiety were described.

Results of the process evaluation were described for both groups.

\section{RESULTS}

Sample

Of the 250 children who had given oral informed consent, 127 were randomly assigned to the experimental group and 123 to the control group. Before the start of the pre-test, 33 children withdrew from the study. Based on the medical examination of the 217 remaining children, another 49 children were excluded, either because the school physician reported secondary headache (27) or because the child had failed to attend the school physician's examination (22). Another 10 children were excluded from the analysis due to a lack of pre-test 
scores ( 8 non-completers and 2 headache-free children). Thus, study results will be presented of 158 children, of which 76 are in the experimental group and 82 in the control group.

The sample consisted of twice as many girls (70\%) than boys and twice as many children (68\%) at elementary school (aged 9-12 years) compared to high school (aged 12-16 years). The mean age was 12.0 (sd 1.9). Almost all children ( $95 \%$ ) had been born in the Netherlands. Headache characteristics of the included children are presented in table 6.2. Both groups were comparable with regard to baseline measures on headache. Scores on depression and trait-anxiety were high, but equal in both groups. The trait-anxiety scores of $52 \%$ of the children in our sample are within the 8 th to 10th deciles of their norm group from the general population. With regard to depression, $39 \%$ of the children were indicated as depressed, including $4 \%$ with scores of 7 or higher.

Table 6.2 Randomization check: comparison of the headache characteristics in the past year on which selection was based, in the experimental $(n=76)$ and control group $(n=82)$

\begin{tabular}{|c|c|c|c|c|}
\hline & & & $\begin{array}{c}\text { Experimental } \\
\text { Group }\end{array}$ & $\begin{array}{l}\text { Control } \\
\text { grroup }\end{array}$ \\
\hline Frequency & $\begin{array}{l}\text { Once a month } \\
\text { Few times a month } \\
\text { Once a week } \\
\text { Few times a week }\end{array}$ & $n(\%)$ & $\begin{array}{c}0 \\
5(7 \%) \\
17(22 \%) \\
54(71 \%)\end{array}$ & $\begin{array}{c}1(1 \%) \\
5(6 \%) \\
17(21 \%) \\
59(72 \%)\end{array}$ \\
\hline Prevalence & $\begin{array}{l}\text { Last week } \\
\text { Today }\end{array}$ & $n(\%)$ & $\begin{array}{l}73(96 \%) \\
53(74 \%)\end{array}$ & $\begin{array}{l}76(93 \%) \\
53(68 \%)\end{array}$ \\
\hline Pain intensity & $\begin{array}{l}\text { Usual pain } \\
\text { Worst pain }\end{array}$ & mean (sd) & $\begin{array}{l}64(19) \\
82(14)\end{array}$ & $\begin{array}{l}65(19) \\
81(17)\end{array}$ \\
\hline Usual dunation & $\begin{array}{l}\leq 1 \text { hour } \\
1 \text { hour }-\frac{1}{2} \text { day } \\
\frac{1}{2}-1 \text { day } \\
1-2 \text { days } \\
>2 \text { days }\end{array}$ & $n(\%)$ & $\begin{array}{l}12(16 \%) \\
13(17 \%) \\
24(32 \%) \\
16(21 \%) \\
11(15 \%)\end{array}$ & $\begin{array}{l}15(19 \%) \\
14(17 \%) \\
22(27 \%) \\
21(26 \%) \\
9(11 \%)\end{array}$ \\
\hline Burden: I feel poin & $\begin{array}{l}\text { Only if I pay attention } \\
\text { But I can ignore it at times } \\
\text { I can do my usual activities } \\
\text { I can only do easy activities } \\
\text { Such that I can't do anything }\end{array}$ & $n(\%)$ & $\begin{array}{c}1(1 \%) \\
8(11 \%) \\
22(29 \%) \\
23(31 \%) \\
21(28 \%)\end{array}$ & $\begin{array}{c}2(2 \%) \\
9(11 \%) \\
22(27 \%) \\
22(27 \%) \\
27(33 \%)\end{array}$ \\
\hline $\begin{array}{l}\text { School absence } \\
\text { Doctor visit }\end{array}$ & $\begin{array}{l}\text { Yes } \\
\text { Yes }\end{array}$ & $\begin{array}{l}n(\%) \\
n(\%)\end{array}$ & $\begin{array}{l}53(71 \%) \\
25(33 \%)\end{array}$ & $\begin{array}{l}54(66 \%) \\
23(28 \%)\end{array}$ \\
\hline
\end{tabular}




\section{Loss-fo-follow-up}

Sample size in both groups at each measurement moment is presented in table 6.3, reflecting compliance to the training and post-test and follow-up measurements. Of the available data at post-test, $11 \%$ was obtained from the retrospective questionnaire used as a proxy for missing diary data. At 3,6 and 12 months follow-up $3 \%, 2 \%$ and $8 \%$ of the data came from the retrospective questionnaire, respectively.

Table 6.3 Sample size in the experimental and attention-control group at pre-test, training compliance, post-test, 3,6 and 12 manths follow-up; with percentages compared to pretest between brackets.

\begin{tabular}{l|ccc|ccc} 
& Pre-test & $\begin{array}{c}\text { Training } \\
\text { compliance }\end{array}$ & Post-test & $\begin{array}{c}\text { After 3 } \\
\text { months }\end{array}$ & $\begin{array}{c}\text { After 6 } \\
\text { months }\end{array}$ & $\begin{array}{c}\text { After } 12 \\
\text { months }\end{array}$ \\
Exp.group & 76 & $60(79 \%)$ & $72(95 \%)$ & $57(75 \%)$ & $56(74 \%)$ & $67(88 \%)$ \\
Cont.group & 82 & $56(68 \%)$ & $70(85 \%)$ & $55(67 \%)$ & $56(68 \%)$ & $60(73 \%)$ \\
\hline
\end{tabular}

All measurements at post-test and follow-up had been completed by $56 \%$ of the children. There were no differences between the experimental and the control group in children who were lost to follow-up and children who completed the assessment at past-test, and at 3,6 and 12 months follow-up, with regard to demographic variables, baseline headache characteristics, trait-anxiety and depression. The process evaluation list had been filled in by $73 \%$ of all children.

Table 6.4 Mean score (standard deviation) at pre-test, sample size ( $n$ ) and mean difference $(\delta)$ (standard deviation) between the pre-test and post-test scores in the experimental and control group, and the $95 \%$ confidence interval of the difference between both groups (group 8), of the primary outcome measures Total Headache Index, headache frequency, intensity, and duration.

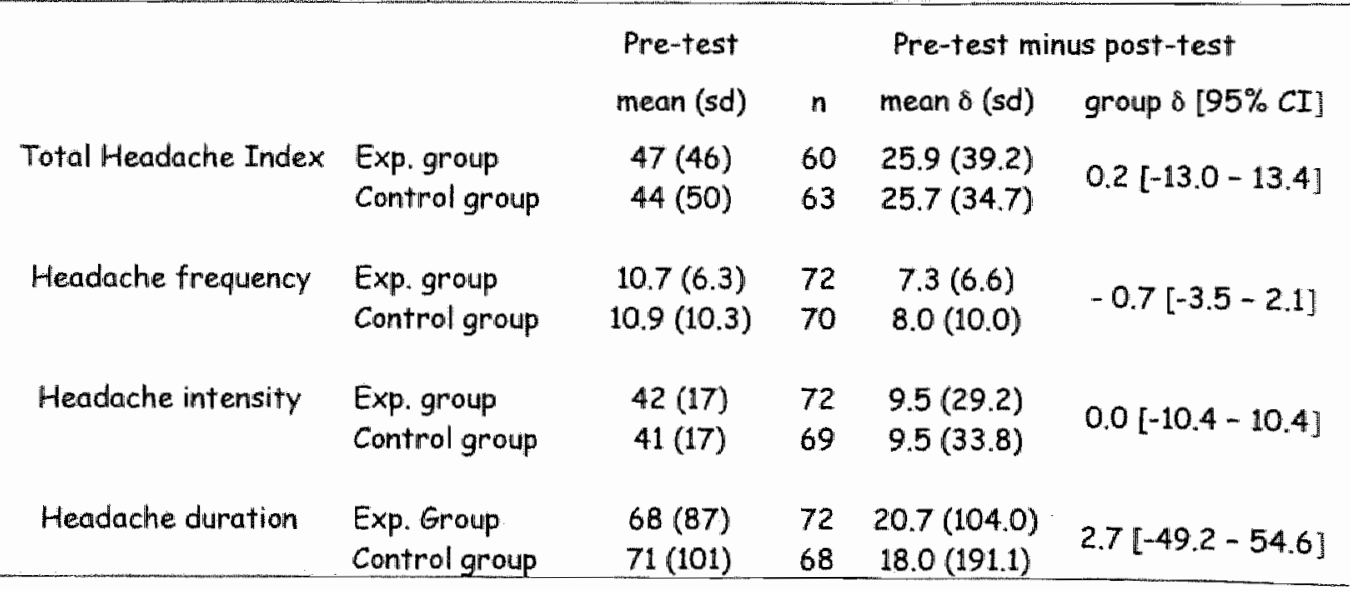




\section{Primary outcome parameters}

Table 6.4 presents the results on the primary outcome parameters comparing pre-test and post-test scores. The two groups did not differ with regard to pretest scores. The mean decrease in headache frequency, intensity, duration and THI is equal in both groups, and the $95 \%$ confidence intervals of decrease difference all include zero. Table 6.5 shows the results at 3,6 and 12 months follow-ups. With one exception on headache intensity at 6 months follow-up, data show no difference between the experimental and control group.

Table 6.5 Mean difference ( 8 ) (standard deviation) and sample size (n) between the pre-test and 3 months follow-up, pre-test and 6 months follow-up, and pre-test and 12 months follow-up, in the experimental (E) and control (C) group, and the $95 \%$ confidence interval of the difference between both groups (group $\delta$ ), of the primary outcome measures Total Headache Index (THI), headache frequency (freq), intensity (int.), and duration (dur.).

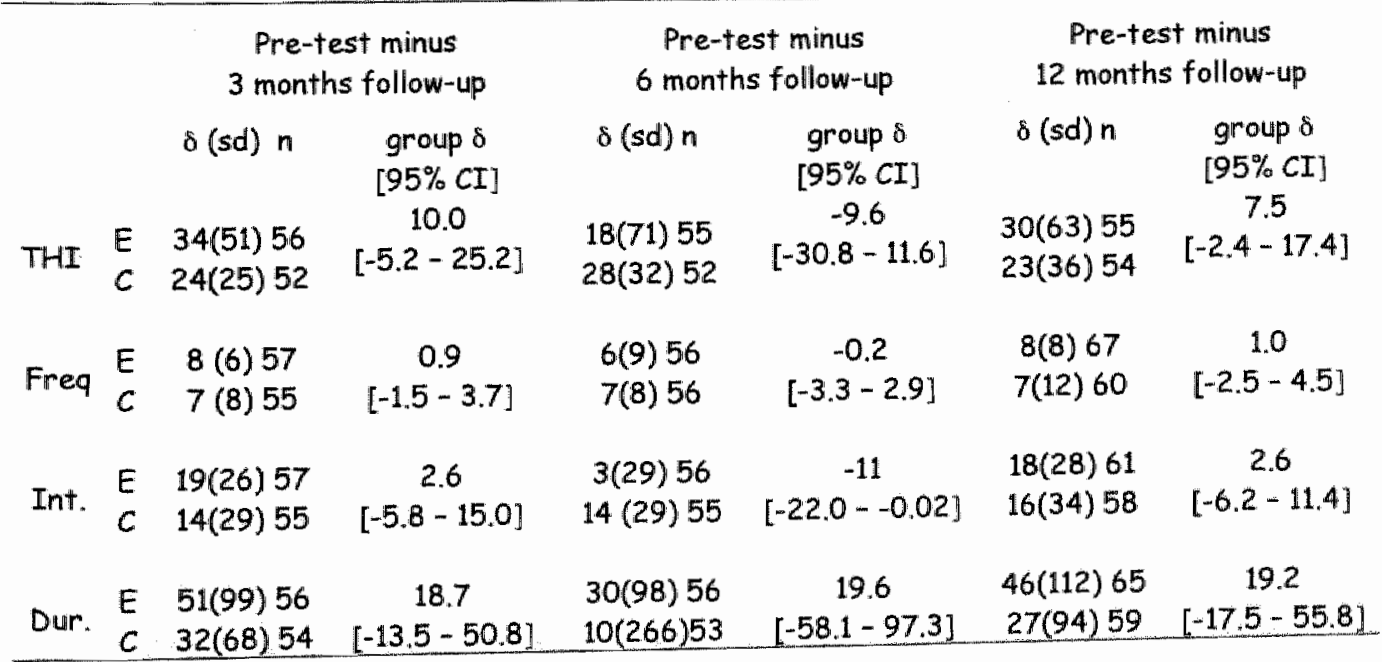

Figure 6.1 is an illustration of the decrease in both groups on frequency, intensity, duration and Total Headache Index. The mean headache frequency in four weeks dropped from 11 to 3 headaches at post-test in both groups. The mean headache intensity decreased from 41 and $42 \mathrm{~mm}$ at pre-test to $32 \mathrm{~mm}$ after the training. Headache duration in four weeks dropped from 68 to 43 hours in the experimental group. In the control group, the mean duration was 71 hours at pre-test and 56 hours at post-test. The mean THI was 47 and 44 at pre-test in the Help Yourself and Learn More group, respectively, and 21 at post-test. 
All results in both groups remain at 3 and 12 months follow-up, with a peak at 6 months follow-up in all parameters in the experimental group, and in duration in the control group.

Figure 6.1 Mean headache frequency, intensity, duration and Total Headache Index in four weeks, comparing experimental and control group, at pre- and posttest and at 3 (fu3), 6 (fu6) and 12 (fu12) months follow-up.

Headache frequency

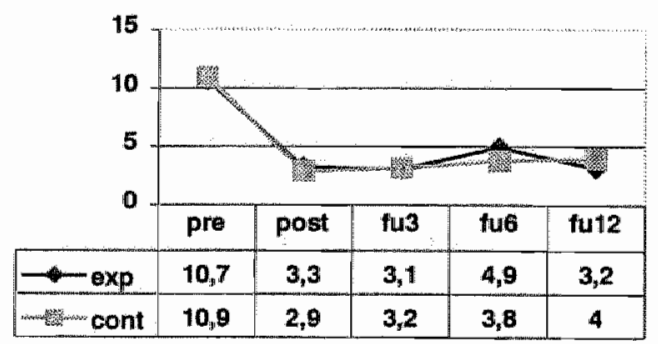

Headache Intensity

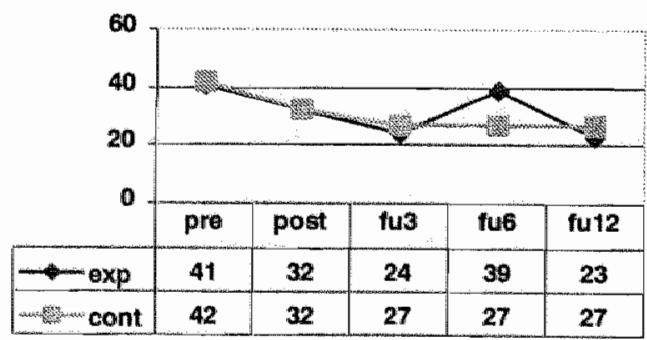

Headache duration

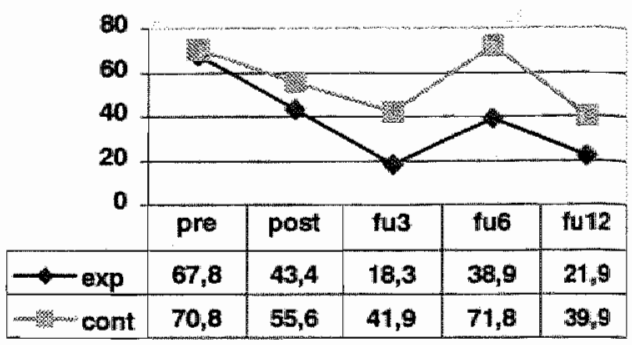

Total Headache Index

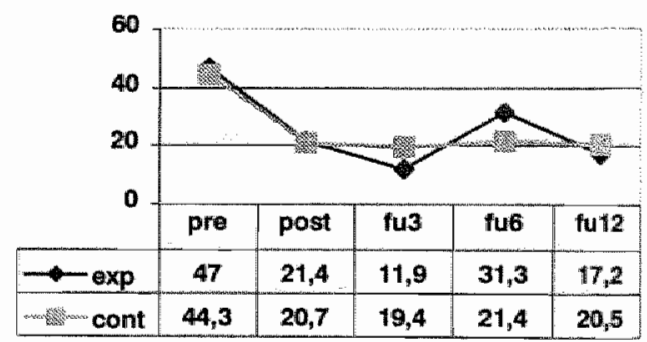

Secondary outcome parameters

In both groups, a high percentage of subjects showed clinically relevant improvements when we compared pre- and post-test scores for frequency (78\%), intensity $(36 \%)$, duration $(66 \%)$, and THI $(71 \%)$. The mean number of headachefree days in four weeks in the experimental and control group increased from 18 up to 24 days at post-test. In each group, $30 \%$ of the children reported complete absence of headache at post-test. 
In general, medication intake, doctor visits and school absence due to headache was low and equal in both groups, and had been reduced at post-test with remaining results at follow-up. Mean medication intake dropped from 1.6 intakes to 0.8 in four weeks, mean number of doctor visits dropped from 0.09 to 0.02 times in four weeks, and school absence reduced from 2.7 hours to 0.4 hours in four weeks. In the experimental group, 27 children took pain medication with a mean of 3.6 times at pre-test. At post-test, 17 children in this group took pain medication with a mean of 3.0 times. In the control group, 45 children used pain medication with a mean of 3.5 times, which reduced to 17 children taking pain medication with a mean of 2.7 times at post-test. Summing both groups during the 20 weeks of assessment at pre- and post-test and follow-ups, a total of 29 visits were paid to the family physician because of headache, by 20 children. In the experimental as well as the control group, 22 children at pre-test and 6 children at post-test reported school absence because of headache. The mean number of hours that these children missed at school was 11 in both groups at pre-test and 6 and 5 hours at post-test in the experimental and control group. respectively.

\section{Additional analysis}

Results in tables 6.4 and 6.5 and figure 1 are comparable, when we only include subjects who had not dropped out once during post-test and follow-ups instead of using an intention-to-treat analysis.

Further analysis encompassed a subgroup that was comparable to the sample of McGrath et al. ${ }^{17} 101$ Children in our sample were aged 11 years or older. Only 3 of them had a pre-test THI score of at least 166, which was the mean THI score of the sample of McGrath. We therefore included all children 11-years of age or older who were within the highest quartile of our THI pretest scores, instead. This subgroup encompassed 26 children: 13 in both groups. There were 19 girls (73\%). The mean THI pre-test score was 90 (sd 34) in the experimental group and 93 (sd 52) in the control group. The mean decrease in THI at posttest was 45 (sd 40) in the experimental and 59 (sd 56) in the control group. The $95 \%$ confidence interval of the difference in decrease (13) between both groups was $[-67-30]$. At post-test, the mean THI was 44 (sd 39) in the experimental and 45 (sd 74) in the control group. At 3,6 and 12 months follow-up, the THI scores were 26 (sd (36), 48 (sd 87) and 21 (sd 31) in the experimental group and 29 (sd 32), 30 (sd 33) and 38 (sd 53) in the control group, respectively.

We found no differences between experimental and control group in pre-test minus post-test scores of frequency, intensity, duration and THI, within boys, 
girls, children at elementary school or at high school, depressed or nondepressed children, or children with high or lower anxiety.

\section{Process evaluation}

Children in the experimental group spent almost 2,5 hours a week [95\% CI: $2.0-$ 2.9] working on the training program, versus 1,5 hours per week [95\% CI: 1.3 1.8] for children in the control group. One quarter of the participants in the Help Yourself group thought that the training program took too much time.

Satisfaction with the results of the training program was comparable between both groups, with a mean and standard deviation of 73.5 (22.5) for the Help Yourself and 70.2 (24.7) for the control group.

Children in the experimental group had most frequently learned how to reduce headache intensity $(71 \%)$, how to reduce headache frequency $(62 \%)$, about their headache triggers $(44 \%)$, and when the headache usually starts $(13 \%)$. Children in the control group had most frequently learned about their headache triggers $(59 \%)$, how to reduce headache intensity $(31 \%)$, how to reduce headache frequency $(29 \%)$, and when the headache usually starts (24\%).

\section{DISCUSSION}

Our study did not show that the "Help Yourself cope with stress and headache" program leads to better results than the Learn More training, that was designed as the attention-control or placebo training. The decrease in headache was similar in both groups. Since a no-intervention control group was not included in the study, we cannot conclude that the experimental as well as the placebocontrol training are beneficial to children with headaches.

Our results do not support the study by McGrath et al., ${ }^{17}$ that showed a positive result for the Help Yourself training when compared to an attention-control group. In their study, the mean THI of 159 in the Help Yourself group had decreased with $50 \%$ at post-test and had stayed almost at baseline level in the control group. The most important difference in the design of both studies, which may account for the different conclusions, concerns the treatment population. McGrath et al. focused on a clinical population of migraine patients who had been referred to a migraine clinic. The population in our study was not clinically referred, but was selected after the administration of a headache questionnaire in a general population of school children. In addition, we included children with primary headaches in general, without specifically diagnosing their headaches as migraine, tension-type headache or both. We merely excluded 
children with secondary headaches. As a result, the baseline headache severity in our sample was significantly lower than in the sample of McGrath et al. Previously, Richter et al. had studied pediatric migraine patients and compared two treatment groups, of relaxation or cognitive coping training, with a placebo control group. ${ }^{35}$ Group differences in treatment effect were found only in children with high headache severity. In children with low headache severity, THI decreased similarly in the training and control groups. In our additional analyses, however, we did not find a difference between both groups in the subsample with the highest headache severity.

Some considerations should be taken into account with regard to the design and execution of our study. The sample size was adequate, although dropout rates were $5 \%-15 \%$ at post-test and $12-33 \%$ at follow-up. Our dropout rates are comparable to those in previous studies. McGrath et al. reported a dropout between $20 \%$ and $35 \%$ in the three groups of the 1988 study and $31 \%$ in the two experimental groups of the 1992 study. ${ }^{17}$ Richter et al. reported an overall dropout of $18 \%$. ${ }^{35}$ Guibert et al. reported $34 \%$ dropout before or during treatment and found that dropouts before the start of treatment experienced less severe headaches and dropouts during treatment were younger. ${ }^{37}$ In our study, dropouts at post-test were comparable to the other subjects, with regard to baseline headache characteristics, demographic variables, and anxiety and depression. Dropouts may inflate results, if dropout is due to lack of treatment success. However, results in our study did not change when we only included subjects who fully completed post-test and follow-ups.

Another point of debate may be the moment of randomization in our study. Contrary to most other studies we randomly assigned subjects to the experimental and control group after the selection and informed consent procedure but before the pre-test assessment. Because an equal amount of children withdrew from the study in both groups, this too early randomization was of no consequence to the sample quality of the experimental and control group.

The content of both the Help Yourself and the control training may be subject to discussion. The Help Yourself training was designed for and tested in children with migraine headaches only. ${ }^{1}$ Although no study has focused on this matter yet, it is not likely that the training is only suitable for migraine headaches and not for other primary headache types. The core focus of the Help Yourself training is on stress-management and stress is viewed as a trigger of migraine as well as tension-type headache, in addition to maintaining and exacerbating the pain. "I 
The control group in our study received a placebo training that was similar in form to the Help Yourself training, but different in content. Evidence of this difference in content is found in the process evaluation of both programs. Significantly more children in the experimental group stated that they had learned how to reduce headache intensity $(71 \%$ versus $31 \%$ ) and headache frequency $(62 \%$ versus $29 \%$ ). Although more children in the control group had learned about their headache triggers $(59 \%)$, the difference with the experimental group $(44 \%)$ isn't all that llarge. Children in the experimental group spent more time on the training program, which did not lead to better results. A placebo-controlled study design has been advocated and used in the field of pediatric headache. ${ }^{17,35,36}$ In two studies statistically and clinically significant differences between therapies and placebo control group were found. ${ }^{17,35}$ Contrary, our study and one on relaxation prophylaxis for childhood migraine ${ }^{36}$ showed an equal effectiveness of experimental and placebo training. So, unfortunately, we still have to cite McGrath et al., ${ }^{36}$ who stated that there is no explanation for these contradictory findings and that the natural variation in children's headaches should be more thoroughly studied. One way to do this is to include a non-treatment control group in effect studies with long-term follow-up assessments, to gain insight in natural variation. Along with ethical problems of not treating headache sufferers for a long period of time, an important problem arises with the measurement and assessment necessary for the scientific evaluation. Headache diaries, instead of retrospective questionnaires or observations by others, have been accepted as the standard for assessing headache in effect studies. ${ }^{3 B}$ However, headache diaries force the subject to focus on headache characteristics and perceived headache triggers, thus changing natural variation.

We conclude with stressing the need for cost-effective treatments for children with headaches in the general population, as well as for clinically referred patients. "Help Yourself" was not more effective than the placebo control training consisting of patient information on headache types and registration of headache triggers. Studies on the natural variation of headache in the general population of children are required. However, new studies of the effectiveness of an even more comprehensive health education program combining ingredients such as patient information, trigger registration, headache monitoring and management strategies may be worth-while. 


\section{REFERENCES}

1. Bandell-Hoekstra $\mathbb{I}_{n}$ Huijer Abu-Saad H, Passchier J. Frederiks C, Feron F, Knipschild P. Prevalence and characteristics of headache in Dutch schoolchildren. European Journal of Pain 2001:5:145-153.

2. Bille B. Migraine in childhood and its prognosis. Cepholalgia 1981:1:71-75.

3. Andrasik F, Passchier J. Tension-type headache, cluster headache and miscellaneous headaches. Psychological aspects. In: Olesen J. Tfelt-Hansen P, Welch KMA, editors. The headoches. New York: Raven Press, 1993, p. 489-492.

4. Passchier J. Andrasik F. Migraine. Psychological factors. In: Olesen J. Tfelt-Hansen P. Welch KMA. editors. The headaches. New York: Raven Press, 1993, pp. 233-240.

5. Peatfield RC, Olesen J. Migraine. Precipitating factors. In: Olesen J. Tfelt-Hansen $P$, Welch KMA, editors. The Headaches. New York: Raven Press, 1993, p. 241-245.

6. Egermark-Eriksson I. Prevalence of headache in Swedish schoolchildren. Acta Paediatr Scond 1982:71:135-140.

7. Maratos J. Wilkinson M. Migraine in childreni a medical and psychiatric study. Cephalalgia 1982;2:179-187.

8. Passchier J, Orlebeke JF. Headaches and stress in schoolchildren: an epidemiollagical study. Cephalalgia 1985;5:167-176.

9. Bandell-Hoekstre I, Huijer Abu-Saad H, Passchier J, Knipschild P. Recurrent headache, coping and quality of life in children: a review. Headoche 2000:40:357-370.

10. Olness $K$, Hall H, Rozniecki JJ, Schmid W. Theoharides TC. Mast cell activation in children with migraine before and after training in self-regulation. Headache 1999:39:101-107.

11. Andrasik F, Gerber WD. Relaxation, biofeedback, and stress-coping therapies. In Olesen J. Tfelt-Hansen P. Welch KMA, editors. The headaches. New York: Raven Press, 1993, p. 833227.

12. Richardson GM, McGrath PJ. Cognitive-behavioral therapy for migraine headaches: a minimal-therapist-contact approach versus a clinic-based approach. Headache 1989:29:352357.

13. Marlowe N. Stressfull events, appraisal, coping and recurrent headache. I Clin Psychol $1998: 54: 247-256$.

14. Turk DC. Meichenbaum D, Genest M. Pain and behavioral medicine: a cognitrive behavioral perspective. New York: Guilford Press, 1983.

15. Engel JM. Relaxation training: a self-help approach for children with headaches, Anter $J$ Occup Ther 1992,46:591-596.

16. Lascelles MA, McGrath PJ, Sullivan MJL. Werk A. Self-administered treatments for adolescents with headache: description, applications, and limitations. Headache Quarterly 1991:2:196-200.

17. MCGrath PJ, Humphreys P, Keene D, Goodman JT, Lascelles MA, Cunningham SJ, Firestone $P$. The efficacy and efficiency of a self-administered treatment for adolescent migraine. Pain 1992:49:321-324.

18. Engel JM, Rapoff MA, Rogot Pressman A. Long-term follow-up of relaxation training for pediatric headache disorders. Headache 1992:32:152-156.

19. Dooley J. Bagnell A. The prognosis and treatment of headaches in children - a ten year follow-up. Can J Neurol Sci 1995:22:47-49. 
20. Attanasio V. Andrasik F, Blanchard EB. Cognitive therapy and relaxation training in muscle contraction headache: efficacy and cost-effectiveness. Headache 1987:27:254-260.

21. Marlowe N. Self-efficacy moderates the impact of stressful events on headache. Headache $1998 ; 38: 662-667$.

22. Larssan, B., Melin, L. and Döberl, A., Recurrent tension headache in adolescents treatted with self-help relaxation training and a muscle relaxant drug. Headache 1990;30:665-671.

23. McGrath PJ، Cunningham SJ, Lascelles MA, Humphreys P. Help Yourself: a treatment for migraine headaches, Patient manual and audiotape. Ottawa: University of Ottawa Press. 1990.

24. Waters WE. Community studies of the prevalence of headache. Headache 1970:9:178-186.

25. Waters WE. The epidemiology of migraine. Bracknell: Boehringer Ingelheim, 1974.

26. Huijer Abu-Saad $H$. Toward the development of an instrument to assess pain in children: Dutch study. Adr Pain Res Ther 1990:15:101-106.

27. Huijer Abu-Soad H, Kroonen E, Halfens R. On the development of a multidimensional Dutch pain assessment tool for children. Pain 1990:43:249-256.

28. Lascelles, M.A., Cunningham, S.J., MCGrath, P., and Sullivan, M.J.L., Teaching coping strategies to adolescents with migraine. J Pain Symptom Management 1989:4:135-145.

29. Richardson GM, McGrath PJ, Cunningham SJ, Humphreys P. Validity of the headache diary for children. Headache 1983;23:184-187.

30. Langeveld JH, Koot HM, Passchier J. Headache intensity and quallity of life in adolescents. How are changes in headache intensity in adolescents related to changes in experienced quality of life? Headache 1997:37:37-42.

31. IHS - Headache Classification Committee of the International Headache Saciety. Classification and diagnostic criteria for headache disorders, cranial neuralgias and facial pain. Cephalalgia 1988;8 (suppl. 7):10-73.

32. Bakker FC, van Wieringen PCW, van der Ploeg HM, Spielberger CD. Handleiding bij de ZelfBeoordelings-Vragenlijst voor kinderen. (Manual of the Dutch State-Trait-AnxietyInventory for Children). Lisse: Swetts \& Zeitlinger, 1989.

33. Spielberger CD, Gorsuch RL, Lushen RE. Manud for the State-Trait Anxiety Inventory for Children (How I Feel Questionnaire). Palo Alto: Consulting Psychologists Press, 1970.

34. De Wit CAM. Depressie uragenlijst voor kinderen. OVK en KOVK. [Depression Questionnaire for children]. Amersfoort/Leuven: Acco, 1987.

35. Richter IL, MoGrath PJ, Humphreys PJ, Goodman JT. Firestone P, Keene D. Cognitive and rellaxation treatment of paediatric migraine. Pain 1986;25:195-203.

36. McGrath PJ, Humphreys P, Goodman JT, Keene D, Firestone P, Jacob P, and Cunningham SJ. Relaxation prophylaxis for childhood migraine: a randomized placebo-controlled trial. Dev Med Child Newrol 1988,30:626-631.

37. Guibert MB. Firestone P. McGrath P. Goadman JT, Cunningham JS. Compliance factors in the behavioural treatment of headache in children and adolescents. Can J Behav $S_{C}$ $1990: 22: 37-44$.

38. Brink $M$ wan den. Bandell-Hoekstra ENG, Huijer Abu-Saad H. The accurrence of recall bias in pediatric headache: a comparison of questionnaire and diary data. Headache 2001:41:1120 . 


\section{Chapter 7}

Help Yourself cope with headache and stress: effects on quality of life and pain coping in children

Bandell-Hoekstra I, Huijer Abu-Saad H, Passchier J, Frederiks C, Feron F, Knipschild P. Help Yourself cope with headache and stress: effects on quality of life and pain coping in children. Submitted. 


\section{ABSTRACT}

"Help Yourself cope with stress and headache" is a home-based, selfadministered training program for pediatric headache, that involves cognitive coping and relaxation. We have studied the effects of "Help Yourself" on changes in Quality of Life (QOL) and use of pain coping strategies. The study sample $(n=158)$ consisted of 9 to 17 year old children from the general population who reported recurrent headaches. In a previous article, we reported no differences in headache outcome, when Help Yourself was compared to a placebo strategy, at post-test and at 3,6 and 12 months follow-up. Now, we did not find differences between groups on QoL improvement or the use of pain coping strategies as well. Within both groups, the QoL sub-domains quality of health and psychological and social functioning were comparably increased, and QoL in general remained the same, after treatment. In addition, QoL worsened with regard to the impact of headache on daily and leisure activities (functional status), and with regard to physical symptoms other than headache. Pain coping strategies were all used less often, except for distraction techniques in the experimental group. 


\section{INTRODUCTION}

There is a growing interest in Quality of Life (QoL) as a major outcome measure of the impact of headache and its treatment, ${ }^{1,2}$ especially in studies that focus on adult migraine patients undergoing pharmacological therapies. The prevalence of recurrent headaches in the general population of children and adolescents is high, ${ }^{3}$ and warrants a focus on headache-related QoL in youth.

QoL is a multidimensional concept that encompasses social, psychological, and physical health. It is personal and subjective, and incorporates positive and negative aspects of well-being. In addition, QoL is a dynamic concept because changes depend on health status. ${ }^{4,5}$ Changes in actual headache presence in adolescents with chronic headache and migraine have an impact on satisfaction with life in general and satisfaction with health. ${ }^{6}$

The relation between headache and QoL is complex and may be influenced by coping. ${ }^{7}$ Coping is an intentional cognitive and behavioral effort to manage stress. ${ }^{8}$ Stress is perceived as an important trigger of headache in children, 3,9 and stress can exacerbate or maintain pain. ${ }^{10}$ The headache pain itself can also be viewed as a stressor that needs to be dealt with. Scientifically evaluated headache treatments in children, including a no-treatment or placebo-treatment control group, are focused on stress and pain management, and include biofeedback, ${ }^{11-13}$ relaxation, ${ }^{14-19}$ relaxation and medication, ${ }^{20}$ self-hypnosis, ${ }^{21}$ cognitive coping, ${ }^{22}$ or a combination of cognitive coping and relaxation training, 23 in addition to biofeedback. ${ }^{24}$ Treatment effects are mainly reported for headache characteristics. So far, no studies have been published on the effect of pediatric headache treatment, that include QoL, pain coping, or both, as outcome measures or mediating variables.

The "Help Yourself cope with stress and headache" training is a 7-week homebased training program, that consists of cognitive and behavioral stress coping and relaxation strategies, with a focus on stress-management as well as pain management. 25 "Help Yourself" is self-administered and thus more costefficient, less threatening and more easily accessible. It was found to be successful in reducing headache in adolescents who were referred to a migraine clinic, when compared to a placebo-control group. ${ }^{23}$ In children and adolescents with frequent headaches from the general population, however, we were not able to replicate these findings. "Help Yourself" did not produce better results in headache change than a placebo-control group. ${ }^{26}$ The same study inventoried QoL and pain coping as well, the results of which will be presented here. 
Aim of the present study is to test the effect of "Help Yourself" on QoL and pain coping. Differences in results between the experimental and control group within sub-groups of the sample will also be described.

\section{METHODS}

\section{Subjects}

Subjects come from a general population of 2,871 school children in grades 6,7 and 8 of elementary schools (aged 9-12 years) and grades 1 to 4 of high schools (aged 12-17 years) in the city and region of Maastricht, The Netherlands. These children have participated in a cross-sectional study, ${ }^{3.27}$ in which they have filled out Waters' Headache Questionnaire (WHQ), 9,28,29 the Pediatric Pain Assessment Tool (PPAT), ${ }^{30,31}$ the Pain Coping Questionnaire (PCQ), ${ }^{32}$ and the QLH-Y." ${ }^{33}$

Independently, two raters scored each child's questionnaire in terms of possible inclusion, combining data on headache frequency, intensity, duration, impact, and perceived headache cause. 433 Children, on whom both raters agreed on inclusion, received an invitational information letter. After a subsequent phonecall, 250 children agreed to participate and were included in the randomization procedure.

\section{Procedure}

The study procedure has been described elsewhere. ${ }^{26}$ The Medical Ethics Committee of the Academic Hospital and University of Maastricht, The Netherlands, approved of the study. It was designed as a patient-blinded Randomized Clinical Trial, involving an experimental group and a control group, with a pre- and posttest and 3,6 and 12 months follow-up.

Both groups received a training program, which was identical with regard to form but different with regard to content. The experimental group received the "Help Yourself cope with stress and headache" training program. ${ }^{23,25,34}$ The control group received a "Learn More" training program, which had been specifically designed as a credible placebo for this study. It encompassed mainly the registration of potential headache triggers.

The programs consisted of one group session on the rationale of the training and baseline assessment of anxiety and depression, four weeks of keeping a headache diary (pre-test), seven weeks of training by means of written information and home assignments, and another four weeks of keeping a headache diary (post-test). 
At the start of each training week, children received by mail a chapter of the training and exercise forms and, in the experimental group, a cassette-tape with relaxation exercises. At pre- and post-test and follow-ups, participants were phoned once for motivation and further instruction on keeping the diary. During the 7 weeks of training, the participants in both groups were phoned once every two weeks, for feedback and motivation.

\section{Measures}

\section{Assessment method}

Quality of Life (QOL) and pain coping were assessed at baseline during the cross-sectional study, and at post-test, and 3,6 and 12 months follow-up.

QoL was assessed using the QLH-Y. ${ }^{33}$ The QLH-Y covers two $0-100 \mathrm{~mm}$ visual analogue scales on Quality of Life in general and Quality of Health, and consists of 69 items comprising 4 sub-domains. Psychological functioning encompasses stress, fatigue, depression, harmony/vitality, cheerful mood and optimism about the future. Physical functioning concerns somatic symptoms other than headache. Social functioning consists of functioning at home and at school, and social interaction with peers and siblings. Functional status covers headache impact on activities of daily life and on leisure activities. Cronbach's alphas of the QLH-Y subscales were between 0.77 and $0.97 .{ }^{27}$ Subscale scores were recoded so that a high score means a high Quality of Life. ${ }^{35}$

Pain coping was assessed with the Pain Coping Questionnaire (PCQ). ${ }^{32}$ The 39 items cover 8 subscales, namely information seeking, problem solving, seeking social support, positive self-statements, behavioral distraction, cognitive distraction, externalizing and internalizing/catastrophizing. Factor analysis on the items of the PCQ confirmed the subscales that were previously reported by Reid et al., ${ }^{32}$ with Cronbach's alphas between 0.78 to $0.87 .{ }^{27}$

A test-retest (after 4 weeks) showed moderate to high reproducibility of the QLH-Y and the PCQ.

Outcome parameters on headache were assessed with a diary, that was kept by the child for four weeks at pre-test and post-test and for two weeks at 3,6, and 12 months follow-up. Trait-anxiety and depression were only assessed at baseline. Trait-anxiety was measured using the ZBV-K, ${ }^{36}$ the Dutch version of the State-Trait Anxiety Inventory for Children. ${ }^{37}$ Depression was measured using the k-DVK, which is a Dutch screening list with 9 dichotomous items on depression. ${ }^{38}$ 


\section{Statistical analysis}

The differences between both groups were described with regard to the change in scores between pre-test and post-test, and pre-test and follow-ups on the sub-domains of the QLH-Y and on the subscales of the PCQ. 95\% Confidence intervals of the difference between groups were calculated. The mean scores at pre-test, post-test, and 3, 6 and 12 months follow-up in both groups were graphically described.

In addition, subgroup analyses were conducted on the results of QoL and pain coping variables at post-test. Several subgroups were defined: boys, girls, elementary school pupils, high school pupils, children who complied with the treatment and children who did not comply (treatment compliance was assessed by counting the number of weeks of returned headache diaries during the training, dichotomized into less than 5 or 5 or more weeks), children with a depression score of less than 7 or 7 and higher, and children with anxiety scores classified according to the norm-group as high (norm-group of $80 \%$ and up) or not. Within these subgroups, the $95 \%$ confidence intervals of the sub-domains of the QLH-y and the subscales of the PCQ, between the experimental and control group at post-test were compared.

Table 7.1 Randomization check: distribution of age and gender, baseline measures on depression, trait-anxiety and headache frequency in the experimental $(n=76)$ and control group $(n=8.2)$.

Gender

Age-group

Depression

Trait-anxiety

Headache frequency
Low
High

Low
High

Boys

Girls

Elementary school

High school

Low ( 47$)$

High ( 27 )

Weekly

Less than weekly
Experimental Group

Control group

$22(29 \%)$

$54(71 \%)$

$26(32 \%)$

$56(68 \%)$

$53(71 \%)$

$23(30 \%)$

$54(66 \%)$

$28(34 \%)$

$42(55 \%)$

$34(45 \%)$

$55(67 \%)$

$27(33 \%)$

$34(45 \%)$

$42(55 \%)$

$42(51 \%)$

40 (49\%)

$70(92 \%)$

$6(8 \%)$
77 (94\%)

$5(6 \%)$ 


\section{RESULTS}

\section{Sample}

Of the 250 participants, 127 children were assigned to the experimental group and 123 to the control group. However, study results will be presented of 158 children: 76 in the experimental and 82 in the control group. Non-inclusion was based on withdrawal before the pre-test $(n=33)$, physiological headache causes reported by the school physician $(n=49)$, and lack of pre-test scores of headache $(n=10)$. Groups were comparable with regard to gender and age, and baseline measures on depression, trait-anxiety and headache frequency (table 7.1), and pre-test scores on QoL and pain coping (see table 7.2 and table 7.4).

At post-test, an equal reduction in headache frequency in four weeks was found in both groups, within most of the children. Headache frequency had increased in only $6 \%$ and had remained the same in $3 \%$. The mean headache frequency in four weeks dropped from 10.7 to 3.3 at post-test in the experimental group, and from 10.9 to 2.9 in the control group. For details, see Bandell-Hoekstra et al. ${ }^{26}$

The mean sample sizes for QoL and pain coping subscales were 75 at pre-test, 60 at post-test, 50 at 3-months follow-up, 51 at 6 months follow-up and 44 at 12 months follow-up in the experimental group, and 76, 57, 45, 49 and 43 in the control group, respectively. Thus, drop-out percentages at post-test and followups range between $20 \%$ and $41 \%$ in the experimental group, and between $25 \%$ and $43 \%$ in the control group. Mean scores of QoL in general and pain coping subscales at baseline, did not differ between children who had and who had not dropped out at post-test. Treatment compliance was good in $79 \%$ and $68 \%$ of the children in the experimental and control group, respectively.

\section{Quality of Life}

Within group comparison shows that after treatment, quality of health, psychological functioning and social functioning improved in both groups, while the evaluation of QoL in general remained comparable. QoL decreased when we looked at the impact of headache on daily and leisure activities (functional status), in particular within the Help Yourself group. A decrease in QoL with regard to physical symptoms other than headache was found in both groups. which means that participants reported more physical symptoms at post-test than at pre-test. Results are presented in table 7.2. We found no differences between the experimental and control group in pre-test - post-test changes of the QoL sub-domains. 
The results at 3,6 and 12 months follow-up are comparable (table 7.3 and figure 7.1), except for social functioning. The improvement in social functioning was a little higher in the experimental group compared to the control group.

Table 7.2 Mean score, standard deviation and $95 \%$ confidence interval at pre-test and posttest, and sample size and mean difference ( $\delta$ ) (standard deviation) between the pre-test and post-test scores in the experimental and control group, and the $95 \%$ confidence interval of the difference between both groups (group $\delta$ ), of the subdomains of the QLH-Y: Quality of Life (QoL), Quality of Health (QaH), psychological functioning, functional status, physical symptoms other than headache, and social functioning.

Pre-test mean (sd) $[95 \% \mathrm{CI}]$
Post-test mean (sd) $[95 \% \mathrm{CI}]$
Post-test minus pre-test $n$ mean $\delta(\mathrm{sd})$ group $\delta[95 \% \mathrm{CI}]^{1}$

$\begin{array}{lcccccc}\text { QoL } & E & 67(30)[64-74] & 69(30)[61-77] & 60 & 2.5(38.1) & -1.1[-14.2-12.0] \\ & C & 69(24)[60-73] & 77(24)[71-83] & 56 & 3.6(33.1) & \\ \text { QoH } & E & 53(34)[45-61] & 77(25)[70-83] & 57 & 23.1(41.2) & 5.6[-8.6-19.7] \\ & C & 63(28)[57-69] & 80(22)[74-86] & 57 & 17.5(34.8) & \\ \text { Psyc E } & 53.7(7.2)[51.9-55.4] & 62.9(17.1)[58.5-67.3] & 55 & 8.8(18.3) & 0.6[-5.7-6.8] \\ \text { func. } C & 56.8(7.2)[55.1-58.4] & 64.4(14.1)[60.6-68.2] & 50 & 8.2(13.9) & \\ \text { Func. E } & 39.1(6.2)[38.4-39.7] & 32.2(6.2)[30.2-34.3] & 35 & -6.8(5.7) & -1.7[-7.4-3.9] \\ \text { stat. C } & 41.7(7.1)[35.9-47.6] & 33.9(7.1)[31.6-36.3] & 32 & -5.1(7.5) & \\ & & & & & & \\ \text { Phys. E } & 23.1(4.0)[22.1-24.0] & 20.5(4.8)[19.3-21.8] & 57 & -2.6(5.9) & 0.1[-1.8-2.1] \\ \text { symp C } & 23.5(3.6)[22.7-24.2] & 20.9(4.3)[19.8-22.1] & 54 & -2.7(4.3) & \\ & & & & & & \\ \text { Soc. E } & 8.8(5.2)[7.4-10.2] & 22.4(6.4)[20.6-24.3] & 37 & 15.3(8.4) & 3.5[-0.1-7.1] \\ \text { func. } C & 9.9(4.3)[8.8-11.0] & 21.4(5.9)[19.7-23.1] & 34 & 11.8(6.7) & \end{array}$

the $95 \%$ confidence intervals all include zero, which means that there is no statistically significant difference between groups 
Table 7.3 Mean difference ( $\delta$ ) (standard deviation) and sample size (n) between the pre-test and 3 months follow-up, pre-test and 6 months follow-up, and pre-test and 12 months follow-up, in the experimental (E) and control (C) group, and the $95 \%$ confidence interval of the difference between both groups (group 8), of the subdomains of the QLH-Y: Quality of Life (QOL), Quality of Health (QOH), psychological functioning, functional status, physical symptoms other than headache, and sacial functioning.

\begin{tabular}{|c|c|c|c|c|c|c|c|}
\hline & & $\begin{array}{l}3 \text { months } \\
\text { minus } p\end{array}$ & $\begin{array}{l}\text { collow-up } \\
\text { e-test }\end{array}$ & $\begin{array}{l}6 \text { months ff } \\
\text { minus pr }\end{array}$ & $\begin{array}{l}\text { ollow-up } \\
\text { e-test }\end{array}$ & $\begin{array}{l}12 \text { months } \\
\text { minus pr }\end{array}$ & $\begin{array}{l}\text { Collow-up } \\
\text { e-test }\end{array}$ \\
\hline & & mean $\delta(s d) n$ & $\begin{array}{c}\text { group } \delta \\
{[95 \% \text { CI }]}\end{array}$ & mean $\delta(s d) n$ & $\begin{array}{l}\text { group \& } \\
{[95 \% \text { CI }]}\end{array}$ & mean $\delta(s d) n$ & $\begin{array}{c}\text { group } 8 \\
{[95 \% \mathrm{CI}]}\end{array}$ \\
\hline QOL & $\begin{array}{l}E \\
C\end{array}$ & $\begin{array}{l}4.8(26.8) 50 \\
9.3(26.9) 46\end{array}$ & $\begin{array}{c}4.5 \\
{[-6.4-15.5]}\end{array}$ & $\begin{array}{l}4.7(32.1) 50 \\
8.1(27.9) 52\end{array}$ & $\begin{array}{c}-3.4 \\
{[-15.3-8.5]}\end{array}$ & $\begin{array}{l}5.6(34.6) 44 \\
-0.2(32.9) 43\end{array}$ & $\begin{array}{c}5.7 \\
{[-8.7-20.1]}\end{array}$ \\
\hline $\mathrm{QOH}$ & $\begin{array}{l}E \\
C\end{array}$ & $\begin{array}{l}15.1(34.0) 51 \\
11.5(35.3) 45\end{array}$ & $\begin{array}{c}3.7 \\
{[-10.5-17.8]}\end{array}$ & $\begin{array}{l}23.1(37.4) 50 \\
13.1(31.7) 48\end{array}$ & $\begin{array}{c}10.0 \\
{[-3.5-23.9]}\end{array}$ & $\begin{array}{c}15.3(47.1) 44 \\
7.1(39.7) 43\end{array}$ & $\begin{array}{c}8.6 \\
{[-10.1-27.2]}\end{array}$ \\
\hline $\begin{array}{l}\text { Psyc } \\
\text { func. }\end{array}$ & $\begin{array}{l}E \\
C\end{array}$ & $\begin{array}{l}14.4(17.8) 46 \\
10.7(13.0) 40\end{array}$ & $\begin{array}{c}3.7 \\
{[-3.0-10.3]}\end{array}$ & $\begin{array}{l}14.6(17.7) 46 \\
10.1(15.8) 42\end{array}$ & $\begin{array}{c}4.5 \\
{[-2.6-1.2]}\end{array}$ & $\begin{array}{c}16.6(15.9) 40 \\
9.8(19.3) 39\end{array}$ & $\begin{array}{c}6.8 \\
{[-1.2-14.8]}\end{array}$ \\
\hline $\begin{array}{l}\text { Func } \\
\text { stat. }\end{array}$ & $\begin{array}{l}\mathrm{E} \\
\mathrm{C}\end{array}$ & $\begin{array}{l}-6.0(4.3) 24 \\
-4.0(6.2) 22\end{array}$ & $\begin{array}{c}-2.0 \\
{[-1.2-5.2]}\end{array}$ & $\begin{array}{l}-5.4(4.9) 26 \\
-6.4(7.0) 23\end{array}$ & $\begin{array}{c}1.1 \\
{[-5.1-7.3]}\end{array}$ & $\begin{array}{l}-5.3(5.8) 16 \\
-4.2(6.4) 20\end{array}$ & $\begin{array}{c}-1.1 \\
{[-5,3-3.1]}\end{array}$ \\
\hline $\begin{array}{l}\text { Phys } \\
\text { symp }\end{array}$ & $\begin{array}{l}E \\
C\end{array}$ & $\begin{array}{l}-0.4(5.5) 48 \\
-2.1(5.3) 43\end{array}$ & $\begin{array}{c}1.7 \\
{[-0.6-3.9]}\end{array}$ & $\begin{array}{l}-1.9(5.2) 48 \\
-2.1(4.7) 46\end{array}$ & $\begin{array}{c}0.2 \\
{[-1.9-2.2]}\end{array}$ & $\begin{array}{l}-0.2(5.7) 42 \\
-1.8(4.5) 43\end{array}$ & $\begin{array}{c}1.6 \\
{[-0.6-3.8]}\end{array}$ \\
\hline $\begin{array}{l}\text { Soc. } \\
\text { func }\end{array}$ & $\begin{array}{l}E \\
C\end{array}$ & $\begin{array}{l}14.7(8.8) 33 \\
12.0(6.6) 29 \\
\end{array}$ & $\begin{array}{c}2.7 \\
{[1.2-6.6]^{*}}\end{array}$ & $\begin{array}{l}16.8(8.4) 29 \\
10.9(8.8) 31\end{array}$ & $\begin{array}{c}5.8 \\
{[1.4-10.3]^{*}}\end{array}$ & $\begin{array}{l}16.1(7.8) 30 \\
11.5(9.3) 28\end{array}$ & $\begin{array}{c}4.6 \\
{[0.1-9.1]^{\star}}\end{array}$ \\
\hline
\end{tabular}


Figure 7.1 Mean scores of the sub-damains of the QLH-Y in the experimental and control group, at pre-and post-test and at 3 (fu3), 6 (fu6) and 12 (fu12) months follow-up.

Qually of Life in general (0-100 VAs)

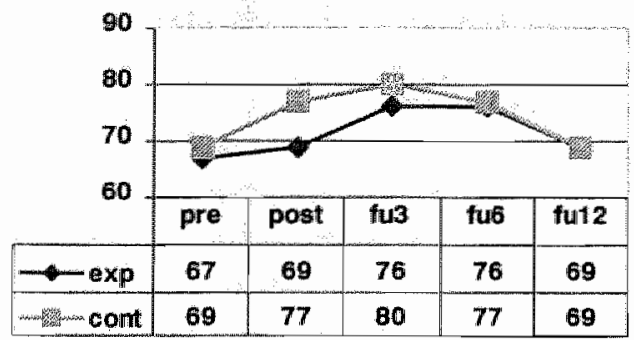

Psychological functioning

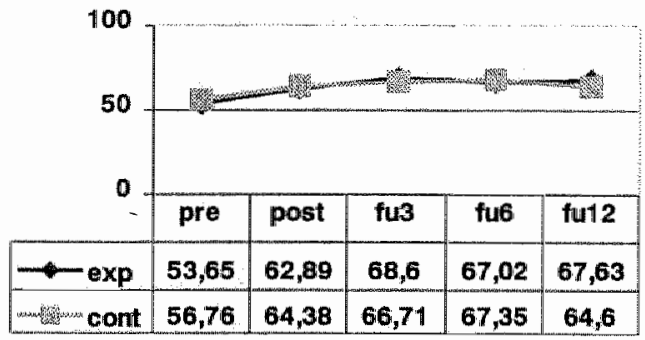

Physical symptoms

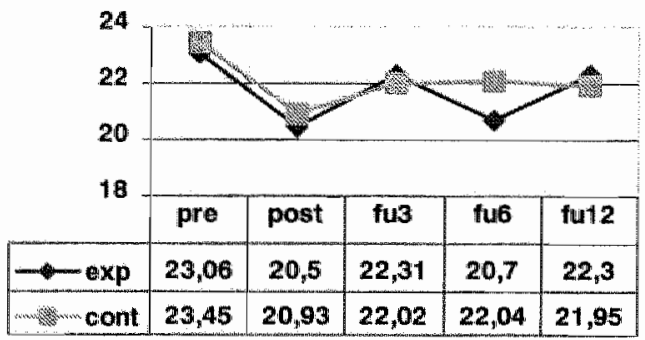

Quality of Health VAS

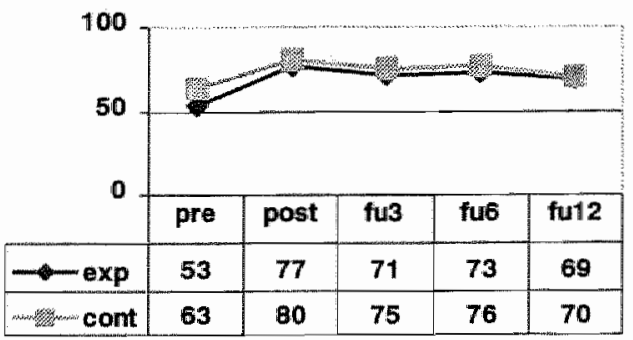

Functional status

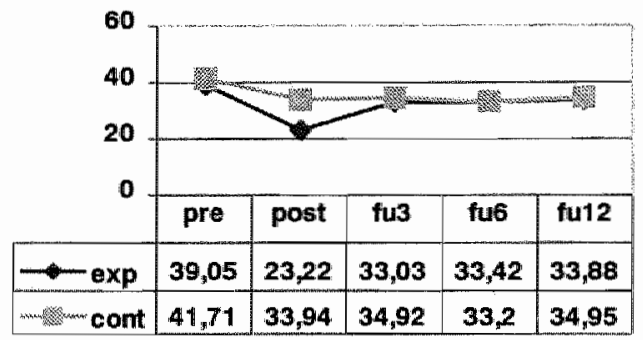

Social functioning

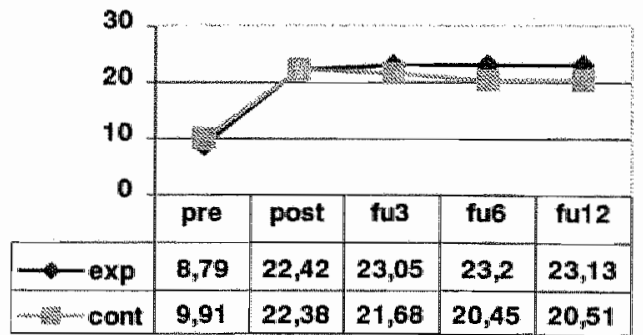




\section{Pain coping}

Table 7.4 shows that the PCQ-scores on problem solving, information seeking, seeking social support, positive self-statements, internalizing and externalizing are all lower at post-test, when compared to pre-test, within the experimental group and the control group. Lower scores indicate that these strategies are used less often. Cognitive and behavioral distraction scores are somewhat lower after the training in the control group, more than in the experimental group. The decrease in use of problem solving and distraction strategies is smaller in the experimental group than in the control group.

Table 7.4 Mean score (standard deviation) at pre-test, sample size ( $n$ ) and mean difference $(\delta)$ (standard deviation) between the pre-test and post-test scores in the experimental and control group, and the $95 \%$ confidence interval of the difference between both groups (group 8 ), of the PCQ subscales.

Pre-test mean (sd) $[95 \% \mathrm{BI}]$

Prob. E $2.3(0.9)[2.1-2.6]$ solv. $\quad C \quad 2.1(0.8)[2.0-2.3]$

Info. $E \quad 1.5(0.5)[1.4-1.7]$ seek. C $1.5(0.7)[1.4-1.7]$

Soc. E $2.3(0.9)[2.1-2.5]$ supp. $\quad C \quad 2.1(0.9)[1.9-2.3]$

Pos. E $2.2(0.8)[2.1-2.4]$ self-st. $C \quad 2.0(0.9)[1.8-2.2]$

Cogn. $E \quad 2.3(0.9)[2.1-2.5]$ distr. $C \quad 2.4(0.9)[2.2-2.6]$

Behav. $E \quad 2.1(0.9)[1.9-2.3]$ distr. C $2.1(0.8)[1.9-2.2]$

Intern. $E \quad 2.5(1.0)[2.2-2.7]$ C $2.4(0.9)[2.2-2.6]$
Post-test mean (sd) [95\% BI] $1.5(0.8)[1.3-1.7]$ $0.9(0.7)[0.7-1.1]$

$0.5(0.5)[0.4-0.6]$ $0.4(0.5)[0.2-0.5]$

$0.9(0.8)[0.7-1.1]$ $1.1(1.0)[0.9-1.4]$

$1.4(0.8)[1.2-1.6]$ $1.1(1.0)[0.8-1.4]$

$2.2(1.0)[1.9-2.4]$ $1.6(0.9)[1.3-1.8]$

$2.0(1.0)[1.7-2.2]$ $1.3(0.9)[1.1-1.5]$
Post-test minus pre-test n mean $\delta(\mathrm{sd})$ group $\delta$ [95\% CI] $6.2-0.9(1.2)$ $57-1.2(0.8)$

$0.3[0.1-0.6]^{*}$

$62-1.1(0.7)$

$57-1.2(0.6)$

$0.1[-0.1-0.4]$

$61-1.3(0.8)$

$57-1.0(1.2)$

$-0.3[-0.7-0.1]$

$61-0.8(1.0)$

$57-0.9(0.9)$

$0.1[-0.3-0.5]$

$61 \quad-0.2(1.0)$

$57-0.9(1.2)$

$0.7[0.3-1.1]^{*}$

$61-0.3(0.9)$

$56-0.8(1.0)$

$0.5[0.2-0.9]^{*}$

$1.0(0.9)[0.8-1.2] \quad 61 \quad 1.4(0.9)$ $0.9(0.8)[0.7-1.1] \quad 56 \quad 1.4(1.0)$
$-0.1[-0.4-0.3]$

$\begin{array}{llllll}\text { Extern. } & E & 1.5(0.6)[1.4-1.7] & 0.6(0.7)[0.4-0.7] & 61 & -0.9(0.7)\end{array}$ $\frac{C \quad 1.5(0.7)[1.3-1.6] \quad 0.5(0.7)[0.3-0.7] \quad 56 \quad-0.9(0.5)}{\text { * the } 95 \% \text { CI does not include zero, which means that there is a statistically significant }}$ $\frac{C \quad 1.5(0.7)[1.3-1.6]}{\text { * the } 95 \% \text { CI does not include zero, which means that there is a statistically significant }}$ $-0.1[-0.3-0.2]$ difference between both groups 
At follow-up (table 7.5 and figure 7.2), results are almost comparable. A difference between groups in mean decrease is only found for cognitive and behavioral distraction at 6 and 12 months follow-up.

Table 7.5 Sample size (n), mean difference ( 8 ) (standard deviation) between the pre-test and 3 months follow-up, pre-test and 6 months follow-up, and pre-test and 12 months follow-up, in the experimental and control group, and the $95 \%$ confidence interval of the difference between both groups (group 8 ), of the PCQ subscales.

\begin{tabular}{|c|c|c|c|c|c|c|c|}
\hline & & \multicolumn{2}{|c|}{$\begin{array}{l}3 \text { months follow-up } \\
\text { minus pre-test }\end{array}$} & \multicolumn{2}{|c|}{$\begin{array}{l}6 \text { months follow-up } \\
\text { minus pre-test }\end{array}$} & \multicolumn{2}{|c|}{$\begin{array}{l}12 \text { months follow-up } \\
\text { minus pre-test }\end{array}$} \\
\hline & & mean $8(s d) n$ & $\begin{array}{l}\text { group } \delta \\
{[95 \% \mathrm{CI}]}\end{array}$ & mean $\delta(s d) n$ & $\begin{array}{l}\text { group } 8 \\
{[95 \% \mathrm{CI}]}\end{array}$ & mean $\delta(s d)$ & $\begin{array}{l}\text { group } \delta \\
{[95 \% \mathrm{CI}]}\end{array}$ \\
\hline $\begin{array}{l}\text { Prob. } \\
\text { solv. }\end{array}$ & $\begin{array}{l}E \\
C\end{array}$ & $\begin{array}{l}-1.1(1.2) 51 \\
-1.2(0.9) 46\end{array}$ & $\begin{array}{c}0.1 \\
{[-0.3-0.5]}\end{array}$ & $\begin{array}{l}-0.9(1.2) 51 \\
-1.2(0.8) 49\end{array}$ & $\begin{array}{c}0.3 \\
{[-0.1-0.7]}\end{array}$ & $\begin{array}{l}-1.4(1.0) 44 \\
-1.2(0.7) 43\end{array}$ & $\begin{array}{c}-0.1 \\
{[-0.5-0.3]}\end{array}$ \\
\hline $\begin{array}{l}\text { Infa. } \\
\text { seek. }\end{array}$ & $\begin{array}{l}E \\
C\end{array}$ & $\begin{array}{l}-1.1(0.6) 51 \\
-1.2(0.7) 46\end{array}$ & $\begin{array}{c}0.1 \\
{[-0.1-0.4]}\end{array}$ & $\begin{array}{l}-1.0(0.6) 52 \\
-1.1(0.6) 49\end{array}$ & $\begin{array}{c}0.1 \\
{[-0.1-0.3]}\end{array}$ & $\begin{array}{l}-1.2(0.6) 44 \\
-1.2(0.7) 43\end{array}$ & $\begin{array}{c}0.1 \\
{[-0.2-0.4]}\end{array}$ \\
\hline $\begin{array}{l}\text { Soc. } \\
\text { supp. }\end{array}$ & $\begin{array}{l}E \\
C\end{array}$ & $\begin{array}{l}-1.0(0.9) 50 \\
-1.2(1.2) 46\end{array}$ & $\begin{array}{c}0.2 \\
{[-0.3-0.6]}\end{array}$ & $\begin{array}{l}-1.0(0.9) 50 \\
-1.0(1.1) 49\end{array}$ & $\begin{array}{c}-0.1 \\
{[-0.5-0.3]}\end{array}$ & $\begin{array}{l}-1.1(1.0) 44 \\
-1.0(1.2) 43\end{array}$ & $\begin{array}{c}-0.1 \\
{[-0.3-0.4]}\end{array}$ \\
\hline $\begin{array}{l}\text { Pos. } \\
\text { self. }\end{array}$ & $\begin{array}{l}E \\
C\end{array}$ & $\begin{array}{l}-1.0(1.2) 50 \\
-1.0(0.9) 46\end{array}$ & $\frac{0.0}{[-0.4-0.5]}$ & $\begin{array}{l}-0.7(1.2) 50 \\
-0.8(0.8) 49\end{array}$ & $\begin{array}{c}0.2 \\
{[-0.3-0.6]}\end{array}$ & $\begin{array}{l}-1.1(1.0) 44 \\
-1.2(0.9) 43\end{array}$ & $\begin{array}{c}0.1 \\
{[-0.4-0.5]}\end{array}$ \\
\hline $\begin{array}{l}\text { Cogn. } \\
\text { dist. }\end{array}$ & $\begin{array}{l}E \\
C\end{array}$ & $\begin{array}{l}-0.5(1.1) 50 \\
-0.8(1.0) 46\end{array}$ & $\begin{array}{c}0.3 \\
{[-0.1-0.8]}\end{array}$ & $\begin{array}{l}-0.3(1.2) 50 \\
-0.8(0.9) 49\end{array}$ & $\begin{array}{c}0.5 \\
{[0.1-1.0]^{*}}\end{array}$ & $\begin{array}{l}-0.4(1.1) 44 \\
-1.0(0.9) 43\end{array}$ & $\begin{array}{c}0.6 \\
{[0.2-1.0]^{*}}\end{array}$ \\
\hline $\begin{array}{l}\text { Beh. } \\
\text { dist. }\end{array}$ & $\begin{array}{l}\mathrm{E} \\
\mathrm{C}\end{array}$ & $\begin{array}{l}-0.5(1.0) 50 \\
-0.9(0.8) 46\end{array}$ & $\begin{array}{c}0.3 \\
{[-0.1-0.8]}\end{array}$ & $\begin{array}{l}-0.4(0.9) 50 \\
-0.8(1.0) 49\end{array}$ & $\begin{array}{c}0.4 \\
{[0.0-0.8]^{*}}\end{array}$ & $\begin{array}{c}-0.3(0.9) \\
-0.1(0.8) 43\end{array}$ & $\begin{array}{c}0.7 \\
{[0.3-1.0]^{*}}\end{array}$ \\
\hline Int. & $\begin{array}{l}E \\
C\end{array}$ & $\begin{array}{l}1.8(1.0) 50 \\
1.5(1.0) 46\end{array}$ & $\begin{array}{c}-0.3 \\
{[-0.7-0.1]}\end{array}$ & $\begin{array}{l}1.7(1.1) 50 \\
1.4(0.7) 49\end{array}$ & $\begin{array}{c}-0.2 \\
{[-0.6-0.2]}\end{array}$ & $\begin{array}{l}1.9(1.2) 44 \\
1.6(1.1) 43\end{array}$ & $\begin{array}{c}-0.4 \\
{[-0.8-0.1]}\end{array}$ \\
\hline Ext. & $\begin{array}{l}E \\
C\end{array}$ & $\begin{array}{l}-1.0(0.8) 50 \\
-0.9(0.6) 45\end{array}$ & {$\left[\begin{array}{c}-0.0 \\
-0.3-0.3]\end{array}\right.$} & $\begin{array}{l}-0.9(0.8) 50 \\
-0.9(0.6) 48\end{array}$ & {$\left[\begin{array}{c}-0.1 \\
{[-0.4-0.2]}\end{array}\right.$} & $\begin{array}{l}1.0(0.7) 44 \\
0.9(0.8) 43\end{array}$ & $\begin{array}{c}-0.1 \\
{[-0.4-0.2]}\end{array}$ \\
\hline
\end{tabular}


Figure 7.2 Mean scores of the subscales of the PCQ in the experimental and control group, at pre- and post-test and at 3 (fu3), 6 (fu6) and 12 (fu12) months follow-up.

Problem solving

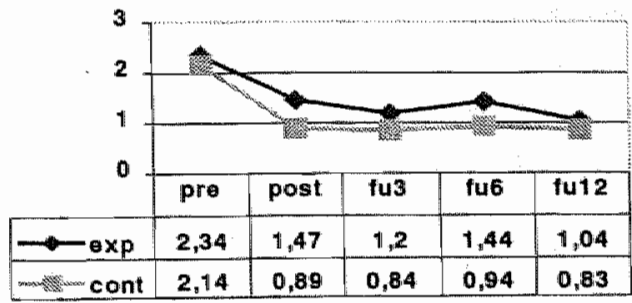

Seeking soclal support

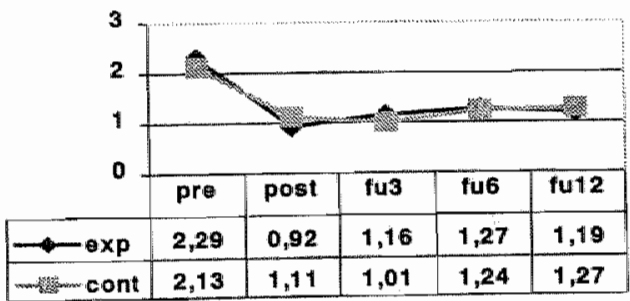

Cognittive distraction

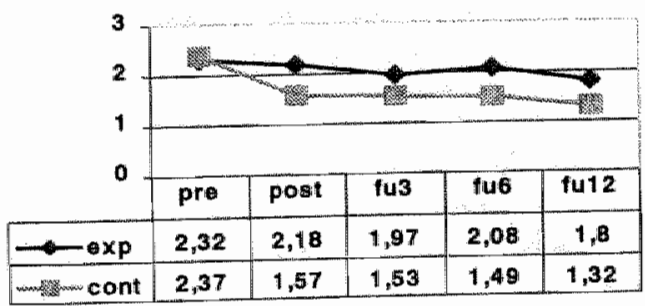

Internaliziling

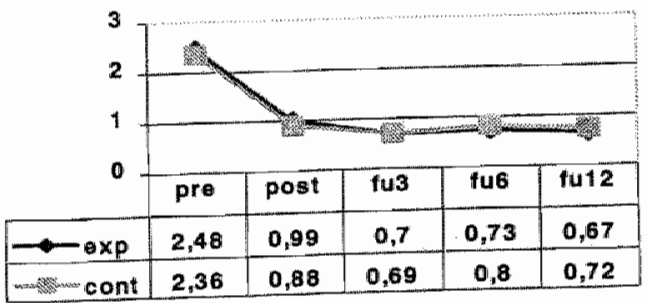

Information seaking

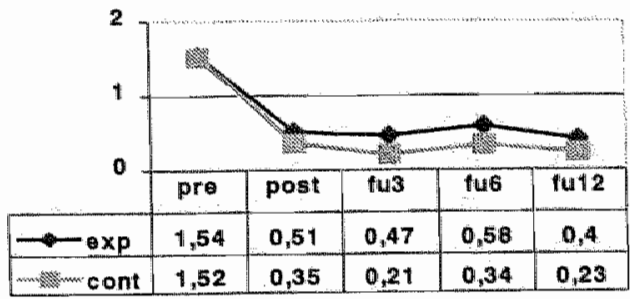

Positive solf-statiments

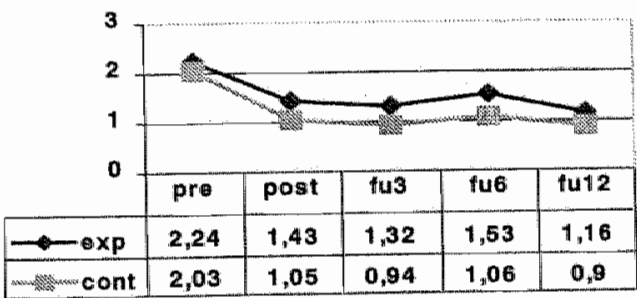

Behaviorall distraction

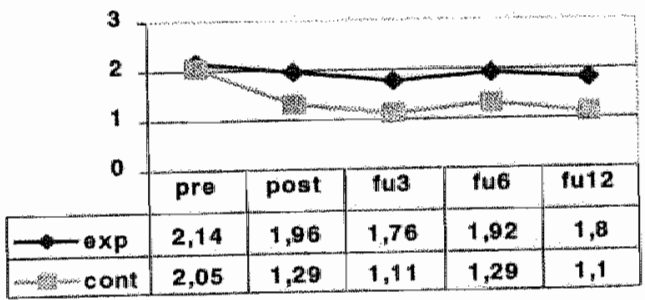

Externalizing

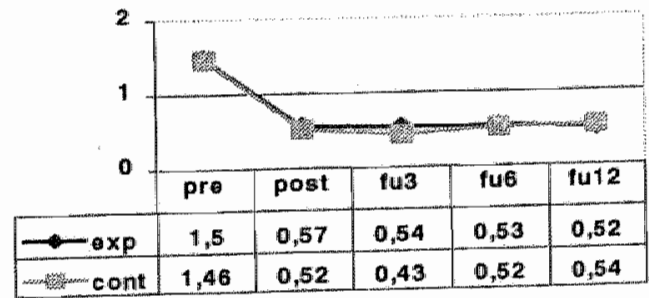




\section{Additional analysis}

Sub-group analysis on the QLH-Y

We found no differences between the experimental and control group with regard to any of the QoL sub-domains at post-test, within boys, within girls, within elementary school pupils, within high school pupils, within children with a high or with a lower score on anxiety, and within children who had and within children who had not completed the training.

Within the group of children scoring high on depression, QoL in terms of functional status was lower at post-test than at pre-test. The mean decrease was higher in the experimental group than in the control group, with a mean of $8.67[-12.15--5.18]$ versus $-1.38[-4.08-1.32]$. There were no differences between the experimental and contral group, within the group of children with a low score on depression.

\section{Sub-group analysis on the $P C Q$}

Boys in the experimental group showed a smaller mean decrease of $-0.19[-0.64-$ $0.26]$ in the use of behavioral distraction techniques at post-test than boys in the control group with a mean of -1.21 [-1.73 - 0.69]. Within girls, there was no difference between both groups.

Within elementary school pupils, we found a smaller mean decrease of -0.16 $[-0.49-0.18]$ in cognitive distraction in the experimental group than in the control group with a mean decrease of -0.92 [-1.32 - -0.52]. Within high school pupils, no differences between the experimental and contral group were found in the change of use of pain coping strategies after treatment.

Children who completed the treatment in the experimental group had a smaller mean decrease in behavioral distraction $(0.29[-1.18-0.63])$ than children who completed the treatment in the control group $(-0.90[-1.18--0.63])$. There was no difference between the experimental and control group on the PCQ subscales within children without treatment compliance.

There was no difference between the experimental and control group on change in PCQ subscale scores at post-test, within children with a high score or within children with a low score on depression, or within the sub-group of children with higher or lower trait-anxiety. 


\section{DISCUSSION}

Our study did not show that the "Help Yourself cope with stress and headache" program results in higher QoL improvement than the Learn More training, that was designed as the attention-control or placebo training. except for social functioning at follow-up. In both groups, quality of health and psychological and social functioning were comparably increased, and QoL in general remained the same, after treatment. In addition, a negative effect was found on QoL with regard to the impact of headache on daily and leisure activities (functional status), and with regard to physicall symptoms other than headache. Gender, age as defined by school type, treatment compliance and anxiety did not contribute to differences in QoL results, between the experimental and control group. Within the sub-group of children scoring high on depression, the negative effect of treatment on functional status was higher in children in the experimental group than in the control group.

In both groups, pain coping strategies assessed by means of the PCQ were used less often than at pre-test. Only the use of cognitive and behavioral distraction techniques remained equal in the experimental group. The two groups differed only with regard to the size of decrease in problem solving and distraction strategies, which was smaller in the experimental than in the control group. Differences in the mean decrease in the use of behavioral distraction between the experimental (small decrease) and control group (larger decrease), were found within boys, and within children who completed the treatment. Differences in the mean decrease in the use of cognitive distraction between the experimental (small decrease) and control group (larger decrease) were found within elementary school pupils.

Previously, withingroup analyses showed positive results with regard to decreasing headache frequency, intensity, duration and index in the Help Yourself training program as well as the Learn More program, within the same study sample. ${ }^{26}$ Current analyses found an increase in Quality of health and psychological and social functioning as well. We stress, however, that there was no difference in change in headache, QoL or pain coping between the experimental and control group.

Moreover, some elements of QoL did not improve after treatment. QoL in terms of headache impact and physical symptoms was lowered. An explanation for this negative result may lay in the difference between baseline and post-test assessment of QoL. Prior to baseline assessment, the child has not specifically focused on headache or QoL. In the weeks before post-test, diary registration and treatment require the child to increase the attention paid to bodily 
sensations and headache impact. In addition, the child may regard the time consumed by study participation as impact of headache on daily and leisure activities. A methodological point for attention is the lack of information on the responsiveness of the QLH-Y questionnaire. We do not know whether the QLH$Y$ is able to detect changes in QoL due to treatment, and do not have any information on the size of detectable changes.

Only one study published so far included $\mathrm{QOL}$ as an outcome measure of a monpharmacological treatment (guided imagery), and aimed at adult tension-type sufferers. No decrease in QoL was found. ${ }^{39}$

Problem solving, information seeking, seeking social support, and positive selfstatements are coping strategies that are all used less often after the Help Yourself training, compared to pre-test. At first sight, these results seem to be contrary to expectations, because Help Yourself is focused on teaching these coping strategies. However, several explanations must be regarded. The first concerns the training itself. The transfer of knowledge may not have been adequately succeeded and the change in behavior may not have been acquired by the child. This may be due to the self-help form of the training, with its relatively short duration, non-committal nature and unverifiable training intensity. Participants spent only 2,5 hours a week working on the training program. ${ }^{26}$ Yet, McGrath et al. have shown equal effectiveness of the selfadministered Help Yourself training, compared to the therapist-administered version. ${ }^{23}$ The second concerns the assessment of pain coping. Assessment at baseline was "out of the blue": there was no prior focus of the child on headache or pain coping, and this may have led to an overestimation of the use of those strategies. Assessment at post-test was after an intensive focus on headache (in the training and diary), which may have resulted in a more realistic estimation of strategy use. The third explanation may be, that the PCQ is not a responsive measurement instrument. There is no data on the ability of the PCQ to assess change in strategy use. The fourth concerns the match between the content of the Help Yourself training and the PCQ. Main focus of the training is on coping with stress. The PCQ, however, is specifically focused on the assessment of pain coping. Treatment effects on stress coping may thus not be assessed by the $P C Q$. The final explanation may be, that expectations of an increase in use of pain coping strategies is incorrect. Due to the reduction in headache frequency, pain coping strategies are used less often. Unfortunately, our results cannot be compared to findings of others, since no other studies have included pain coping as an outcome measure in (non-pharmacological) treatment studies, yet. Future studies should consider these speculations. 
A few other methodological aspects need to be considered. The number of statistical tests in our study may have resulted in an increased chance of finding one or more statistically significant differences between groups, which are actually false. With problem-solving at post-test as the one exception, however, the differences found are all within the same sub-scales and in the same direction. The clinical relevance of the rather small difference in change in social functioning between both groups may be a matter of debate. The difference in the use of distraction strategies appears to be more clinically relevant.

The dropout rates in QLH-Y and PCQ assessments ranging between $20 \%$ and $43 \%$ at post-test and follow-ups are high and a cause for concern with regard to the validity of the study. A shortened version of both questionnaires may be required to facilitate a higher response rate within clinical studies.

The population in our study was not a clinically referred population, but was selected after the administration of a headache questionnaire in a general population of school children. Focus is on primary headaches in general, achieved by excluding children with secondary headaches, but without specifically diagnosing their headache. Headache severity at pre-test, as measured using the Total Headache Index, was low when compared to the sample of migraine patients in the study of McGrath et al. ${ }^{23,26}$ Knowledge is lacking of the influence of a relatively low headache severity on the effectiveness of Help Yourself with regard to $Q 0 L$ and pain coping variables.

In conclusion, we failed to show that the Help Yourself training program is more effective than a placebo-control training in reducing headache, improving $Q \mathrm{OL}$ and increasing the use of pain coping strategies. However, we would like to stress the importance of QoL as an outcome measure in studies on headache. The inclusion of pain coping as a mediating, moderating or outcome variable in the evaluation of treatments focusing on changing pain coping strategies, such as cognitive-behavioral training programs, should become a rule, rather than an exception.

\section{REFERENCES}

1. Lipton RB. Health-related Quality of Life in headache research. Editorial. Headache 1995;35:447.

2. Solomon GD. Evolution of the measurement of Quality of Life in migraine. Neurology 1997:48:510-15. 
3. Bandell-Hoekstra I, Huijer Abu-Saad H, Passchier J, Frederiks C, Feron F, Knipschild P. Prevalence and characteristics of headache in Dutch schoolchildren. European Journal of Pain 2001:5: 145-153.

4. Bowling A. Measuring disease. Buckingham: Open University Press, 1995.

5. Schipper H. Quality of Life. Principles of the clinical paradigm. I Psychosoc Oncal 1990;8:171-185.

6. Langeveld JH, Koot $H M$, Passchier $J$. Headache intensity and quality of life in adolescents. How are changes in headache intensity in adolescents related to changes in experienced quality of life? Headache 1997:37:37-42.

7. Bandell-Hoekstra I, Huijer Abu-Saad H, Passchiler J, Knipschild P. Recurrent headache, coping and quality of life in children: a review. Headoche 2000;40:357-370.

8. Lazarus RS. Emotion and Adaptation. New York: Oxford University Press: 1991.

9. Passchier J, Orlebeke JF. Headaches and stress in schoolchildren: an epidemiological study. Cephalalgia 1985;5:167-176.

10. Andrasik F, Gerber WD. Relaxation, biofeedback, and stress-coping therapies. In: Olesen $J$, Tfelt-Hansen P, Welch KMA, editors. The headaches. New York: Raven Press, 1993, p. 833227.

11. Fentress DW, Masek BJ, Mehegan JE, Benson H. Biofeedback and relaxation-response training in the treatment of pediatric migraine. Dev Med Child Neuro/1986;28:139-146.

12. Labbé EE. Treatment of childhood migraine with autagenic training and skin temperature biofeedback: a component analysis. Headache 1995;35:10-13.

13. Olness K, Hall H, Rozniecki JJ, Schmidt W, Theoharides TC. Mast cell activation in children with migraine before and after training in self-regulation. Headache 1999:39:101-107.

14. Engel JM, Rapoff, MA. A component analysis of relaxation training for chillren with vascular, muscle contraction and mixed-headache disorders. In: Tyler DC, Krane EJ (Eds.). Adwaces in Pain Research Therapy. Vol. 15. New York: Raven Press, 1990, p. 273-290.

15. Engel JM, Rapoff MA, Rogot Pressman A. Lang-term follow-up of relaxation training for pediatric headache disorders. Headache 1992;32:152-156.

16. Lar'sson $B$, Melin L. Chronic headaches in adolescents: treatment in a school-setting with relaxation training as compared with infarmation-contact and self-registration. Pain $1986: 25: 325-336$.

17. Larsson B, Daleflod B, Häkansson L. Melin L. Therapist-assisted versus self-help rellaxation treatment of chronic headaches in adalescent: a school-based intervention. $J$ Child Psychol Psychiat 1987:28:127-136.

18. McGrath PJ. Humphreys P, Goodman JT, Keene D, Firestone P, Jacob P, Cunningham SJ. Relaxation prophylaxis for childhood migraine: a randomized placebo-contralled trial. Dev Med Child Neurol 1988;30:626-631.

19. Passchier $J_{n}$ van den Bree MBM, Emmen HH, Osterhaus SOL, Orlebeke JF, Verhage F. Relaxation training in school classes does nat reduce headache complaints. Headache $1990: 30: 660-664$.

20. Lar'sson B, Melin L. Döberl A. Recurrent tension headache in adolescents treated with selfhelp relaxation training and a muscle relaxant drug. Headache 1990:30:665-671.

21. Olness K. MacDonald JT, Uden DL. Comparison of self-hypnosis and propranollol in the treatment of juvenile classic migraine. Pediatrics 1987;79:593-597.

22. Richter IL, McGrath PJ, Humphreys PJ, Goodman JT, Firestone P, Keene D. Cognitive and relaxation treatment of paediatric migraine. Pain 1986:25:195-203. 
23. McGrath PJ, Humphreys P, Keene D, Goodman JT, Lascelles MA, Cunningham SJ. Firestane $P$. The efficacy and efficiency of a self-administered treatment far adolescent migraine. Pain 1992:49:321-324.

24. Osterhaus SOL. Passchier J, van der Helm-Hylkema $H$, de Jong KT, Orlebeke JF, de Grauw AJC, Dekker PH. Effects of behavioral psychophysiological treatment on schoolchildren with migraine in a nonclinical setting: predictors and process variables. I Ped Psychol $1993 ; 18: 697-715$.

25. McGrath PJ, Cunningham SJ, Lascelles MA, Humphreys P. Help Yourself: a treatment for migraine headaches, Patient manual and audiotape. Ottawa: University of Ottawa Press. 1990.

26. Bandell-Hoekstra I, Huijer Abu-Saad H, Passchier J, Frederiks C, Feron F, Knipschild P. Effects of a self-help program in a general population of children with frequent headaches. Submitted. (chapter 6 of the dissertation).

27. Bandell-Hoekstra I, Huijer Abu-Saad $H$, Passchier J, Frederiks C, Feran F, Knipschill P. Coping and Quality of life in relation to headache in Dutch schoolchildren. European Jaurnal of Pain 2002:6:315-321.

28. Waters WE. Community studies of the prevalence of headache. Headache 1970:9:178-186.

29. Waters WE. The epidemialogy of migraine. Bracknell: Boehringer Ingelheim, 1974.

30. Huijer Abu-Saad $H$. Toward the development of an instrument to assess pain in children: Dutch study. Adv Pain Res Ther 1990:15:101-106.

31. Huijer Abu-Saad $H$, Kroonen $E$, Halfens R. On the development of a multidimensional Dutch pain assessment tool for children. Pain 1990:43:249-256.

32. Reid GJ, Gilbert CA, MCGrath PJ. The Pain Coping Questionnaire: preliminary validation. Paing 1998: 76:83-96.

33. Langeveld JH, Koot HM, Loonen CB, Hazebroek-Kampschreur AAJM, Passchier J. A Quality of Life instrument for adolescents with chronic headache. Cephalalgia 1996:16:183-196.

34. Lascelles, M.A., Cunningham, S.J., McGrath, P., and Sullivan, M.J.L., Teaching coping strategies to adolescents with migraine. J. Pain Sympt Manag 1989:4:135-145.

35. Langeveld JH, Koot HM. Passchier J. Do experienced stress and trait megative affectivity maderate the relationship between headache and Quality of Life in adalescents? I Ped Psychol 1999:24:1-11.

36. Bakker FC, van Wieringen PCW, van der Plloeg HM, Spielberger CD. Handleiding bij de ZelfBeoordelings-Vragenlijst voor kinderen. (Manual of the Dutch State-Trait-AnxietyInventory for Children). Lisse: Swets \& Zeitlinger, 1989.

37. Spielberger CD, Gorsuch RL, Lushen RE. Manual for the State-Trait Anxiety Inventory for Children (How I Feel Questionnaire), Palo Alto: Consulting Psychologists Press, 1970.

38. De Wit CAM. Depressie vragenlijst voor kinderen. DVK en KDVK. [Depression Questionnaime for children]. Amersfoort/Leuven: Acco, 1987.

39. Mannix KL. Chandurkar RS, Rybicki LA, Tusek DL, Solomon GD. Effect of guided imagery on Quality of Life for patients with chronic tension-type headache. Headache 1999:39:326334. 
Chapter 8

DISCUSSION AND CONCLUSION 


\section{INTRODUCTION}

Headache is a public health problem because of its prevalence and impact on daily life. Most people deal with their headaches themselves. Education, with emphasis on developing self-management skills to prevent headache attacks and to alleviate the pain, must begin early, in order to prevent the development of a chronic or recurrent pain syndrome.

The aim of the research described in this thesis was to gain insight into headache prevalence, headache-related pain coping and Quality of Life (QoL) in the general population of school-aged children, and to test the short- and longterm effect of a cognitive-behavioral training program focused at teaching selfmanagement skills to children with frequent headaches.

Two studies were conducted: a cross-sectional study on headache, pain coping and QoL in children at elementary and high school, and a randomized trial, comparing the "Help Yourself cope with stress and headache" training program with a placebo program.

This chapter presents a short summary of the major findings of each study. Detailed discussion of theoretical and methodological issues can be found in earlier chapters. The focus here is on the major issues that need to be addressed. The chapter will finish with concluding remarks on the results presented in this thesis, together with implications for clinical practice, and further research.

\section{THE CROSS-SECTIONAL STUDY}

\section{What were the research questions?}

The cross-sectional study investigated the prevalence of headache in school children in the Netherlands, and compared the findings of the current study with a study conducted in the early 1980s. Of particular interest was whether or not the prevalence of headache had increased, and if children were able to accurately recall prior headache complaints. Furthermore, the difference in the use of pain coping strategies and QoL between children with low, medium and high headache severity was assessed.

\section{What were the major findings?}

The prevalence of headache in children between the ages of 9 and 17 years was high. Half of all children experience a headache at least ance a month, including $22 \%$ weekly. Severe weekly headaches were reported by $12 \%$ of the children. 
When compared to $1983^{1}$, the prevalence of weekly headaches in 10-17 year olds has increased by $6 \%$, from $17 \%$ to $23 \%$.

The results from our cross-sectional study were supported by the comparison of the questionnaire data of a sub-group of children with data from a headache diary. At group level, the median score of headache frequency was equal on the questionnaire and the diary. The correlation between both assessment methods, which is an indication of intra-individual agreement, was however, low. Headache intensity and duration were strongly overestimated on the questionnaire.

Headaches were considered to be severe if children experienced weekly headaches that lasted more than an hour and were painful with an intensity of 50 or more on a 0-100 visulal analogue scale. Migraine-related characteristics, such as unilateral headache pain, headache onset in the morning or upon waking, accompanying symptoms such as nausea, vomiting and photophobia and phonophobia, and precipitating warning signals were found most frequently within this group, when compared to children with medium or low headache severity. Children with the most severe headaches often considered stress to be a trigger of their headaches. School absence due to headache was reported by more than two-thirds of these children, compared to one-fifth of the children with infrequent, short-lasting and low intensity headaches. Selfreported consultation of a (family) physician because of headaches was $9 \%$ overall, and $30 \%$ for the children with severe headaches.

Children with the highest headache severity reported the lowest QoL in general and the lowest Quality of Health, as well as the most prablems with regard to physical functioning, impact of headache on daily and leisure activities, physical symptoms other than headache, and social functioning at home. With regard to using pain coping strategies, children with the most severe headaches more often saught social support, but they used distraction techniques and "seeking information" less often, and they catastrophized and externalized more often.

\section{Are the findings of headache prevalence internationally comparable?}

In comparison to similar self-report studies conducted in the 1990's, the current Dutch figures on weekly headaches are equal to those in Iceland (22\%) ${ }^{2}$, a little lower than those in Australia $(29 \%)^{3}$ and the USA $(30 \%)^{4}$ and much higher than those in Sweden $(6 \%){ }^{5}$

We included 2815 school children between the age of 9 and 17 years. Participant selection was based on sampling at school level, i.e., all pupils in the same elementary school or in the same grade at high school were asked to participate. The questionnaire was administered in the classroom by the researcher. The focus of the study was specifically on headache. 
Waters Headache Questionnaire was used in its original Dutch translation. First, children were asked whether they had ever experienced a headache, and then, whether they had experienced a headache in the past year. If so, children were asked to record how often they had experienced a headache in the past year: once, a few, once a month, several times a month, once a week, or several times a week.

In Iceland, 2140 children aged 11-12 and 15-16 were asked how often they had experienced a headache, with the response categories: never or rarely, at least once a month, once a week, more often than once a week, almost daily. The question was part of a nationwide survey on the health behavior of school children. The questionnaire was administered in the classroom, but only to randomly selected children.

In Australia, 900 school children between the age of 10 and 18 years filled out a questionnaire on headache activity, self-statements and coping responses that had been specifically developed by the researchers. Administration was for all pupils who agreed to participate, during class. Children needed to estimate how often on average they experienced headache: never at all, once a month, once every 2-3 weeks, once a week, once every few days, or almost all the time. The higher prevalence of weekly headaches, when compared to our results, cannot be explained by differences in study methods.

In the USA, a nationally representative sample of 6072 adolescents aged between 11 and 21 were interviewed at home. The answers on the question "how often have you had a headache during the past 12 months?" were graded: never, just a few times, about once a week, almost every day, every day. The rather large interval between "just a few times" and "about ance a week" could be an explanation of the higher prevalence of weekly headaches.

In Sweden, a stratified sample of 1144 pupils aged between 7 and 16 years filled out a questionnaire on headache at home, by themselves or with the help of parents. The first question was: "Do you often have a headache?". If the answer was affirmative, the next question was directed at the frequency: once a year. once a month, several times a month, once a week, several times a week or daily. Children with headaches associated with trauma, infectious disease and fever were excluded based on the questionnaire, and, apparently, without considering the possibility of experiencing both primary and secondary headaches. The much lower rate of $6 \%$ reported in the Swedish study is suspected to be an underestimation because of the first "selective" question, and because of the rigorous exclusion of children who reported secondary headaches. 
Our study design is comparable to studies that report similar results of weekly headaches in school children. This international comparability supports the external validity of the study.

\section{Do more and more children really suffer from headaches?}

In 1983, one in six Dutch children experienced weekly headaches, compared to one in five in 1995. No further replication studies on headache in the general population of school children in the Netherlands have been reported. The hypothesis of an increase in headache prevalence was based on the results of a physical examination of 7 year olds in 1974 and $1992^{6}$ and was supported by our study. From this, two questions arise. Firstly, if there is an actual increase in the prevalence of primary headaches, what has caused the increase? And, secondly, what factors contribute to a higher report of frequent headaches, in case the hypothesis of an actual increase is not true?

If there is an actual increase in primary headaches, the following explanation is commonplace. Nowadays, children experience more stress than 10 or 20 years ago. Life is more demanding, more focused on achievements at school, sports and hobbies, and the number of choices and opportunities is overwhelming. Scientific studies to support this line of thought are lacking. Another possible explanation may lie in the prevalence of unhealthy eating habits: $13 \%$ of $11-12$ year alds and $25 \%$ of $13-15$ year olds skip their breakfast or eat inadequately. ${ }^{7}$ A US study showed an increase in adults skipping breakfast between 1965 and $1991 .{ }^{8}$ Possibly, this trend can be found in children as well. The lack of food and fluid intake may trigger headache later on in the day.

If the increase in primary headaches is only an increase in report instead of an increase in actual prevalence, two possible explanations arise. Firstly, cultural differences in complaint behavior may exist over time. Perhaps it became more and more customary between the 1980's and the 1990's to express feelings of discomfort and to admit that you suffer from headaches. In fact, these cultural differences may even exist between the two cities, namely Amsterdam (1983) and Maastricht (1995). Secondly, both Dutch studies relied on children's selfreport. Rigorous exclusion of secondary headaches by means of physical examinations, a thorough history taking and prospectively assessed information by means of a headache diary, was not part of the previous and the current cross-sectional study. Perhaps secondary headaches were not always recognized as such by the respondents. If that's the case in the 1983 and the 1995 study, then an actual increase in hidden secondary headaches may have become manifest as an increase in primary headaches. For instance, headaches related to allergies may not be recognized as such and the child may consider them as 
primary headaches. Several studies in different countries indicate an increase in respiratory allergies over the past 10-25 years. ${ }^{9-13}$

Thus, a conclusion on the increase of more frequent headaches must be tentatively drawn.

What is the value of the cross-sectional study, if it is so difficult to think back accurately on headache characteristics?

Recall errors are a potential limitation of cross-sectional studies that rely on the self-report of a subject, where the subject, adult or child, is asked to think back to experiences in the past. Many factors, related to the complaint and to the psychological state and personal variables of the subject, contribute to recall errors. The combination of all these factors determines the recollection of the complaint and may lead to an under- or overestimation. The study reported in chapter five indicated that recall errors in our cross-sectional study were related to age and headache severity. Older children appeared to be less accurate in their pain reports on a questionnaire; they showed a tendency to underestimate their headache frequency and to overestimate their pain intensity. Children with severe headaches tended to overestimate the frequency of their headaches, but to give a more accurate report on headache intensity.

In general, the comparison of questionnaire and diary data showed that despite the intra-individual variability in responses, the questionnaire estimation of headache frequency at group level seemed to be accurate.

The strength of cross-sectional studies is that they are practical and economical, especially when a large sample size is required that stems from the general population. Problems with referral bias, within the health care system or due to self-referrals, are bypassed. In addition, headache and pain are subjective in nature and self-report presumably is the most valid and reliable method of assessment, not only in adults but in children as well.

\section{Can we conclude that severe headaches cause a low Quality of Life?}

An important aim of our study was to gain insight into headache as a public health problem in children. With regard to headache and QoL, the findings are informative because they come from a study with a large sample size, focused on the general population of school children. Findings with regard to differences in Qol between children with low, medium and high headache severity are intuitively appealing. However, the cross-sectional study design does not establish the direction or causality of the relationships between headache and QoL, and as such, does not contribute to conclusions on the conceptual madel that was presented in chapter two. 


\section{What is the relationship between coping and headache?}

As in headache and QoL, the same caution should be exercised when concluding on the relationship between headache and coping, based on a cross-sectional study design. It appeared however, that children with the most severe headaches used distraction techniques less often than other children. Cognitive and behavioral distraction strategies are usually thought of as healthy, and most non-pharmacological treatments are focused on teaching these strategies (table 1.3). Insight in the use of "healthy" and "unhealthy" coping strategies may direct future therapies.

In the field of headache, coping needs to be well defined. The conceptual model of headache, coping and QoL (figure 2.1) shows two distinctive features of coping: stress coping and pain coping. Coping with stress relates to coping with a major headache trigger. Healthy stress coping strategies can reduce the occurrence of headache. Pain coping can be viewed as coping with the pain of an already existing headache. In other words, the aim of the pain caping strategies is to reduce the pain intensity and duration.

In the cross-sectional (chapter four) and the intervention study (chapter seven) pain coping, not stress coping, was assessed and included as a research variable. Measurement of stress coping is difficult, because stress is subjective and situation-specific, and so is stress coping. Although the same goes for pain coping, at least the situation to which pain coping refers, namely headache, is clear. Undoubtedly, a deeper understanding of stress coping and headache is needed in order to fully appreciate the complexity of these relationships.

\section{What could have been done differently in the study?}

The sampling procedure could be criticized. The study sample was a convenience sample, instead of a (stratified) random sample of the total population. All eligible schools were contacted for participation and all schools willing were included in the sample. Therefore, the sample was constructed at school level, instead of at an individual level. Children in the same class-room or at the same school may share characteristics that influence headache occurrence (school building, school-related stress, social economic status, etc.). In order to consider these aggregated characteristics, multi-level analysis would have been required. ${ }^{14}$

The classification of headache severity into low, medium and high, and subsequent comparisons of estimated mean and $95 \%$ confidence intervals was an arbitrary choice. The definition of the three groups may be questioned as well as the inclusion of a medium group in the comparisons. 


\section{THE RANDOMIZED TRIAL}

\section{What was the research question?}

The randomized trial focused on the question: Is the "Help Yourself" training program more effective on headache, pain coping and QoL than a control training program, in children of the general population with frequent primary headaches?

\section{What were the major findings?}

We selected children who indicated the highest headache severity on the headache questionnaire in the cross-sectional study and offered them to participate in a randomized trial aimed at reducing their headache complaints.

We compared 76 children who participated in the "Help Yourself cope with stress and headache" training program with 82 children who participated in the placebo-control treatment, i.e. the "Learn More" training program on headache triggers. The randomized trial showed no differences in treatment effect on headache, pain coping or QoL. We found a similar decrease in headache activity within the experimental and control group. In other words, irrespective of the type of treatment, i.e. "Help Yourself" or placebo, many children appeared to benefit.

\section{Are the findings in line with other studies?}

The findings are not in line with the work of the author of the Help Yourself training. McGrath found that Help Yourself was more effective than a placebocontrol training, and equally effective but more cost-efficient than the same treatment administered by a therapist. ${ }^{15}$ The most important explanation of the difference in study results lies in the choice of the treatment population. Our research focused on a non-clinical population of children with frequent primary headaches. McGrath et al. focused on a clinical population of migraine patients who had been referred to a migraine clinic. As a result, the baseline headache severity in their sample was significantly higher. In addition, a clinical population suggests not only more severe headaches but also longer lasting complaints. The participants in our study were selected based on their reports in the crosssectional study, which was a 'one-off snapshot' of their health. They were selected at a moment in their lives where their headaches happened to be severe, and the "natural" direction in the course of their headaches would probably be improvement.

The Help Yourself training program was evaluated in Germany as well, ${ }^{16}$ although results have not been published in a scientific journal so far. Information on their study methods and results are based on personal communication (a paper). 
They included 35 children with headaches at least twice a month. Participants were children from secondary schools, but sampling methods, headache characteristics and randomization procedures are unclear. Findings show that $65 \%$ of the children in the experimental group reached clinical improvement (of more than $50 \%$ ) versus $27 \%$ of the children in the "own best effort" group. The control group was asked to look for possible headache triggers, but it is not clear whether this control "treatment" can be viewed as a placebo with methods similar to the experimental group. The limitations with regard to sample size and the lack of information on study procedures further complicate a comparison with our findings.

In conclusion, in contrast with two other studies, one aimed at a clinical study population with more severe migraine headaches and one small, unpublished study with limited information on study methods, our findings do not support a positive effect of the "Help Yourself cope with stress and headache" training program.

\section{Did we use the proper treatment?}

As in other psychological treatment programs for headache, the Help Yourself training program is based on the assumption that heightened levels of stress are associated with an increased headache activity. The rationale behind the Help Yourself training program is to provide children with a variety of stress coping strategies in order to maximize the probability that they will learn a strategy that is best suited to their individual coping styles. ${ }^{17}$ The Help Yourself training program was found to be effective in adolescents with migraine, as were other self-administered relaxation programs. ${ }^{15,18,19}$ The choice for the Help Yourself training program was therefore evidence based.

The question "did we use the proper treatment?" could be rephrased as "did we use the proper treatment within the proper population?" Compared to the population aimed at by MCGrath ${ }^{15}$, our population was characterized by a low headache severity of a less chronic nature. The results within the control group may direct a focus on patient self-education with regard to headache, by means of diary registration, first, within the general population, before adopting a therapeutic stress-reduction approach.

\section{Did we use the treatment properly?}

The self-help characteristic of the experimental treatment suggests an active involvement in and own responsibility for treatment participation of the child. Measures were taken to enhance the active approach and to motivate the child. An informative group session was held at the start of the study. Children were phoned at home, once every two weeks, by the researcher or the research 
assistant "to whom the children of both groups had been randomly assigned. Telephone protocols had been developed and were pre-discussed and evaluated by the researcher and the research assistant, in order to promote an equal approach of the children and their questions.

Nonetheless, subjects in trials are rather autonomous and tend to deviate from the experimental protocols. ${ }^{20}$ These deviations are difficult to assess. Deviations by means of co-interventions, such as taking medication or seeking medical help, were scarce and equally distributed between both groups. Treatment deviation was $21 \%$ in the experimental group, if non-compliance is viewed as such. Compliance classification was based on the number of weeks of not-returned headache diaries, which may be regarded as a rather imprecise estimation of compliance.

Deviation from the protocol may also refer to investing less time than required in reading and exercising. Children in the experimental group spent approximately 2,5 hours a week on the Help Yourself training program, which is a little more than 20 minutes a day. Twenty minutes is presumably too short for filling out the headache diary, reading the chapter, practicing the relaxation exercises and reflecting on cognitive strategies. Comparison with previous studies is impossible because process evaluations are lacking.

The process evaluation did not include a test of knowledge and skill improvement. Based on the amount of time spent on the training program, we hypothesize that the cognitive and behavioral skills of stress management may not have been internalized well enough.

Why did we compare the experimental with a placebo-control group?

The choice of a control group is either no-treatment or placebo-treatment. Positive effects in a treatment group compared to a no-treatment control group only show that "something" is better than "nothing". A no-treatment control group is valuable in exploring hypothesized beneficial treatment effects, without distinguishing between the specific and non-specific treatment elements.

Non-specific elements refer to the placebo effect, the Hawthorne effect, the Pygmalion effect, the novelty effect, and social desirability. Actual ineffective treatments may result in symptom- or disease improvement, due to the positive expectations of the patient or therapist (placebo), the recognition of the health problem and the attention provided by the therapist (Hawthorne), the selffulfilling prophecy of the unconscious influence of the research and the researcher on the therapist and the patient (Pygmalion), the (temporary) attractiveness of innovative treatments and new technologies (novelty), and 
social desirability resulting in pre-test over-reporting and post-test underreporting of complaints. ${ }^{21}$

Scientific studies aim to control the non-specific elements in order to study the specific treatment effects. A placebo-control group is then imperative. ${ }^{20}$ A notreatment control group could be added, in order to achieve control over the natural course of headache. However, the no-treatment control group should also be a no-assessment control group, which is highly unlikely in scientific studies.

The dispute about the placebo control group stems from the search for a balance between science and clinical practice. In contrast to the scientific need for controlling the placebo and other non-specific effects, the need in clinical practice is to maximize the placebo effect. In clinical practice, the positive effect of the treatment, irrespective of the specific or non-specific elements, should be as large as possible. ${ }^{21}$

\section{Did we use the proper placebo treatment?}

Dependent on the results of a study, this question will come up in two ways. In case the experimental treatment shows positive results, the main question will be: was the placebo treatment good enough to be a "true" placebo? In case positive results are lacking, the question will be: was the placebo treatment too good?

Was the placebo good enough? A true placebo mimics as many aspects of the treatment under study, except for the specific therapeutic elements. The specific therapeutic element of the Help Yourself treatment was the training in cognitive-behavioral coping strategies (including relaxation exercises). Except for the cassette tapes, we incorporated the form of the Help Yourself training program into the placebo treatment. Researcher-patient contacts were therefore comparable. The difficulty in the construction of a psychological placebo treatment lies in its content, which requires that the placebo is unspecific, yet plausible. The focus on headache triggers, "Learn More" in our study, appeared to meet these criteria. Treatment satisfaction was fairly high in both groups (74 and 70 on a 0-100 VAS).

Was the placebo too good? Based on the study results that showed an equal improvement in headache complaints, one is tempted to answer this question affirmatively: the Learn More training program consists of specific therapeutic elements. In other words, the mere writing down of what you eat and drink, the temperature in your classroom, your sleeping habits and activity pattern, sensory perceptions, and stressors, without any information on what to do with the registered headache triggers, is viewed as therapeutic. The need for 
registration requires a shift in the focus of the child towards its headache, and facilitates communication. It needs to be stressed, however, that registration of perceived headache triggers was conducted in both groups by means of the headache diary. In the control group, the focus on triggers was merely enlarged. The process evaluation confirmed this assumption, by showing that although more children in the control group had learned about their headache triggers. this difference wasn't all that large ( $59 \%$ versus $44 \%$ ). Moreover, even when a specific therapeutic effect of trigger registration exists, one would expect the therapeutic effect of the specific elements of Help Yourself (cognitive strategies and relaxation exercises) to be higher.

Since the study did not include a second control group that received notreatment at all, the assumption of a specific therapeutic effect of the control treatment cannot be tested. In fact, the quality of the placebo treatment should be established a priori. Development of process criteria of a priori quality approval of the form and content of placebo treatment is needed to guide future studies. Additionally, the positive results in the control group should not be considered merely as a placebo effect. The same considerations as for the non-specific elements of the Help Yourself training program apply, including the natural course of headache, the regression to the mean effect and measurement errors due to social desirability.

\section{Did we use correct statistical methods?}

Two important choices were made with regard to the statistical analysis of the randomized trial, namely "intention-to-treat" analysis and summarizing the abserved data using confidence intervals.

Intention-to-treat is the idea that subjects should be analyzed as a member of the group to which they were randomly assigned, even if they did not receive the intended treatment, or only a portion of it. Intention-to-treat can be opposed to "treatment-received" analysis and to "adherers only". ${ }^{20}$ Intention-totreat analyses are logically related to randomization. Randomization prevents the influence of unknown factors such as selection bias and confounding. Deviation from the "intention-to-treat" principle partly undoes the advantages of randomization. ${ }^{21}$ The results in chapter six showed no differences between analyses that were intention-to-treat or adherers only.

Currently, statistical inferences using point estimates and confidence intervals are preferred to hypothesis tests. Confidence intervals convey useful information regarding the precision of the estimate. P-values are inadequate for summarizing data, because they do not convey the magnitude of a clinical effect. The size of the $p$-value is a consequence of the magnitude of the observed 
treatment difference and the variability belonging to it. The observed variability is a consequence of sample size. Thus, the size of the experiment contributes to a portion of the $p$-value. The $p$-value also hides the size of the treatment difference. Instead of a focus on the $p$-value, focus should be on the size and clinical significance of an observed treatment effect. ${ }^{20}$

\section{What could have been done differently in the study?}

The choice of the study population, namely children from the general population, has influenced the baseline headache severity and consequently reduced the chances of finding a positive treatment effect. Following scientific rules, we should have thrived for a more homogeneous sample, following rigid selection criteria. Our approach was more practical, aimed at a primary health care setting, and therefore reaching out to the general population. A more homogeneous sample, including only migraine patients, for instance, would have resulted in a higher headache severity at pre-test, but would have disregarded the headache problem in the general population.

A methodological point of debate is the moment of randomization, which was before the pre-test. The disadvantage of (too) early randomization is the inclusion of early failures (withdrawals), which increases the required sample size. In addition, a meaningful interpretation of results is hampered, because a substantial number of the randomized subjects did not receive the assigned treatment. From a statistical point of view, late randomization is thus preferred. The generalizability of results in studies with late randomization, however, may be put up for debate. ${ }^{22}$

\section{CONCLUDING REMARKS}

\section{Conclusion}

The studies that have been presented in this thesis shed light on the scope of the headache problem in the population of school children in general. One in four to five children experience weekly headaches and severe weekly headaches are reported by $12 \%$. In these children, QoL is diminished. In addition, they use "healthy" coping strategies, such as cognitive and behavioral distraction, less often than children with less severe headaches, and "unhealthy" coping strategies, such as catastrophizing, more often. The Help Yourself training program, which aims at the education of cognitive-behavioral coping strategies, resulted in headache improvement and partly changed QoL in children with the most severe headaches from the general population. However, the same results 
were found in children who participated in the control group. Therefore, we cannot conclude that the Help Yourself training program is effective in reducing headache, in children of the general population.

\section{Clinical implications}

More positive results of the Help Yourself program would have simplified the matter of clinical relevance of our study and would have easilly generated implications for clinical practice. For now, clinical relevance lies in spreading the conclusion of this thesis into clinical practice.

\section{Remaining research questions}

Several questions remain open for future study. The associations between headache, pain coping and QoL are still hypothetical and need more prospectively designed studies. More information is needed on the questionnaires: the content validity of the Pain Coping Questionnaire (PCQ) and the responsiveness of both the PCQ and the Quality of Life Headache in Youth (QLH-Y) questionnaire. In addition to pain coping strategies, stress coping needs to be addressed. New studies on the effectiveness of a more basic, comprehensive health education program, combining ingredients of the Help Yourself and of the control treatment program, such as patient information, trigger registration, headache monitoring and management strategies, may be worth-while in the general population. A replication of our randomized trial could be conducted in a population of migraine patients. A more thorough process evaluation, which encompasses a test of change in knowledge and behavior, would be advocated. The development of procedural guidelines to establish the quality of a placebo treatment 'a priori' would be useful.

\section{REFERENCES}

1. Passchier J, Orlebeke JF. Headaches and stress in schoolchildren: an epidemiologal study. Cephalalgia 1985:5:167-176.

2. Kristjánsdóttir $G$ "Wahlberg $V$. Sociodemographic differences in the prevallence of selfreparted headache in Icelandic school-children. Headache 1993:33:276 -280.

3. King NJ, Sharpley CF. Headache activity in children. Paediatr Child Health 1990:26:50-54.

4. Rhee H. Prevalence and predictors of headaches in US adollescents. Headache 2000:40:528. 538.

5. Carlsson J. Prevalence of headache in schoolchildren: relation to family and schoolfactors. Acto Paediatr 1996;85:692-696.

6. Sillanpää $M$. Anttila P. Increasing prevalence of headache in 7-year-old schoollchildmen. Headache 1996:36:466-470. 
7. Poort EC, van der Wal MF. Uitenbroek DG, Pauw-Plomp H. Verschillenin ontbijtgewoonten bij schoolkinderen van Nederlandse, Surinaamse, Marokkaanse en Turkse herkomst. TSG 2001;79:150-154.

8. Haines PS, Guilkey DK, Popkin BM. Trends in breakfast consumption of US adults between 1965 and 1991. J Am Diet Assoc 1996:96:464-470.

9. Anthracopoulos M, Karatza A, Lialios E, Triga M, Triantou K. Priftis K. Prevalence of asthma among schoolchildren in Patras, Greece: three surveys over 20 years. Thorax $2001.56: 569$ 571.

10. Downs SH, Marks GB, Sporik R, Belosouva EG, Car NG, Peat JK. Continued increase in the prevalence of asthma and atopy. Arch Dis Child 2001;84:20-23.

11. Kuehni CE, Davis A, Brooke AM, Silverman $M$. Are all wheezing disarders in very young (preschool) children increasing in prevalence? Lancet 2001:358:846.

12. Ronchetti R, Villa MP, Barreto M, Rota R, Pagani J, Martella S, Falasca C, Paggi B, Guglielmi $F$, Ciofetta $G$. Is the increase in childhood asthma coming to an end? Findings from three surveys of schoolchildren in Rome. Eur" Respir J 2001:17:881-8:86.

13. Wal MF van der, Uitenbroek DG, Verhaeff AP. [Increased proportion of elementory school children with asthmatic symptoms in the Netherlands, 1984/85-1994/95; a literature review.] Ned Tijdschr Geneeskd 2000,144:1780-1785.

14. Courgeau D, Baccaini B. Multilevel analysis in the social sciences. [Analyse multi-niveaux en sciences sociales.] Population 1997:52:831-863.

15. McGrath PJ, Humphreys P, Keene D, Goodman JT, Lascelles MA, Cunningham SJ, Firestone $P$. The efficacy and efficiency of a self-administered treatment for adolesecent migraine. Pain 1992;49:321-324.

16. Luka-Krausgrill U, Kötting K, Kerbeck K. Haerkötter C. Headache in children: one-year follow-up of the "Help Yourself" program. Poster presentation (\#34) at the Fourth International Symposium on Pediatric Pain. Helsinki, Finland, June 29 - July 4, 1997.

17. Lascelles MA, McGrath PJ, Sullivan MJL, Werk A. Self-administered treatments for adolescents with headache: description, applications and limitations. Headache Quarterly, Current treatment and research 1991;2:196-200.

18. Larsson B, Daleflod B, Hakansson L, Melin L. Therapist assisted versus self-help relaxation treatment of chronic headaches in adolescents: a school-based intervention. Journal of Child Psychology, Psychiatry and Allied Disciplines 1987:28:127-136.

19. Larsson $B$, Melin L. Lamminen M. Ullsted F. A school-based treatment of chronic headaches in adolescents. Journal of Pediatric Psychology 1987:12:553-566.

20. Pilantadosi 5. Climical trials. A methodalagical perspective. New York: John Willey \& Sons, 1997.

21. Bouter LM, van Dongen M.CJM. Epidemiologisch onderzoek. Opzet en interpretatie. Utrecht: Bohn, Scheltema \& Holkema, 1988.

22. Durrleman S, Simon R. When to randomize? J Clin Oncol 1991;9:116-122. 
SUMMARY 


\section{SUMMARY}

Headache in children is commonly reported and, as in adults, under-diagnosed and under-treated in the general population. The focus is on primary headaches, without distinguishing between migraine and tension-type headache. The aim of the thesis was to gain insight into headache prevalence, headache-related pain coping and Quality of Life in the general population of school-aged children, and to test the short- and long-term effect of a cognitive-behavioral training program teaching self-management skills, for children with frequent headaches. To this end, we conducted a cross-sectional study on headache, pain coping and Quality of Life in 2815 schoolchildren, followed by a randomized trial.

Chapter 1 gives a definition of headache and describes the complexity of the problem of headache within the general population. The major research variables have been restricted to headache, pain coping and Quality of Life.

Chapter 2 aims at the clarification of the concepts of pain coping and Quality of Life (QoL) and presents a literature review on the strategies that children with recurrent headaches use to cope with their pain, the impact of recurrent headaches on children's QoL, and the influence of personal and situational variables on headache, coping and QoL in children.

Chapter 3 and chapter 4 report on the results of the cross-sectional study. Results showed weekly headaches in one in five boys and one in four girls at elementary school (10-12 years of age), and one in seven boys and one in three to four girls at high school (12-17 years of age). When compared to figures from a previous study in the Netherlands published in 1985, the prevalence of weekly headaches in 10-17 year olds was increased by $6 \%$. Children with low, medium and high headache severity differed with respect to all headache characteristics, such as pain quality, accompanying symptoms, warning signals, location, onset, impact of headache, family occurrence, perceived cause, medical consultation, and school absence. Children with the highest headache severity reported the lowest Quality of Life in general and the lowest Quality of Health, as well as the most problems with regard to physical functioning, impact of headache on daily and leisure activities, physical symptoms other than headache, and social functioning at home. With regard to using pain coping strategies, children with the most severe headaches looked for more social support, internalized and externalized more, used less behavioral and cognitive distraction techniques, and sought information less. 
Chapter 5 compares headache reports in the cross-sectional study with headache reports in the randomized trial, within a sample of 181 children who suffered from headaches frequently. Compared to the report in the headache diary, headache intensity and headache duration were overestimated on the questionnaire. At group level, headache frequency was comparable between both assessment methods.

Chapter 6 and chapter 7 evaluate the "Help Yourself cope with stress and migraine" training program, within a preventive youth health care setting focused on the general population of schoolchildren in the Netherlands. Subjects $(n=158)$ have been randomly assigned to the experimental Help Yourself group or the placebo-control group. Headache frequency, intensity, duration and Total Headache Index (THI) at pre-test were compared with posttest and 3,6 and 12 months follow-up assessments. Although headache frequency reduced from 11 to 3 headaches in four week, no differences were found between both groups. In addition, no differences between groups were found on QoL or the use of pain coping strategies. Within both groups, the QoL sub-domains quality of health and psychological and social functioning were comparably increased, and QoL in general remained the same, after treatment. QoL worsened with regard to the impact of headache on daily and leisure activities, and with regard to physical symptoms other than headache. Pain coping strategies were all used less often, except for distraction techniques in the experimental group.

Chapter 8 concludes on both studies and discusses the methods and results of the cross-sectional study and the randomized trial. 
SAMENVATTING 


\section{SAMENVATTING}

Ook al wordt hoofdpijn bij kinderen niet altijd onderkend en vaak onvoldoende behandeld, toch komt het meer voor dan veelal wordt gedacht. Dit proefschrift richt zich in principe niet op kinderen met dusdanig ernstige hoofdpijnklachten, dat zij uitgebreid onderworpen zijn aan medische diagnostiek en behandeling. Het richt zich op "gewone" kinderen die last van hoofdpijn hebben, of anders gezegd: op kinderen die "gewoon" last van hoofdpijn hebben. Er wordt dan ook geen poging gedaan om deze hoofdpijn in te delen in migraine of spanningshoofdpijn.

Doel van het proefschrift is om aan de hand van een dwarsdoorsnede-onderzoek inzicht te krijgen in de volgende vragen. Hoe vaak komt hoofdpijn bij kinderen voor? Hoe gaan kinderen om met hoofdpijn? En wat is de kwaliteit van leven van kinderen met veel hoofdpijn in vergelijking tot kinderen met weinig hoofdpijn? Minstens zo belangrijk is om uit te zoeken wat je eraan kunt doen. Door middel van een gerandomiseerde interventiestudie is het effect onderzocht van een "Help Jezelf" (omgaan met stress en hoofdpijn) training gericht op cognitieve vaardigheden en ontspanningstechnieken.

Hoofdstuk 1 definieert hoofdpijn en beschrijft hoofdpijn als probleem in de algemene populatie. Omdat hoofdpijn een complexe aandoening is, zijn de belangrijkste variabelen van dit proefschrift beperkt tot hoofdpijn, pijn coping en kwaliteit van leven.

Hoofdstuk 2 is een literatuuroverzicht waarin de begrippen pijn coping en kwaliteit van leven worden verhelderd en worden uitgewerkt in relatie tot hoofdpijn bij kinderen. Ook worden persoons- en situatiegebonden variabelen die van invloed zijn op hoofdpijn, pijn coping en kwaliteit van leven nader beschouwd. Hoofdstuk 3 en hoofdstuk 4 beschrijuen de resultaten van het dwarsdoorsnedeonderzoek. In groep 7 en 8 van de basisschool rapporteert één op de vijf jongens en één op de vier meisjes wekelijkse hoofdpijn. In het voortgezet onderwijs, tot en met klas 4, komt wekelijkse hoofdpijn voor bij één op de zeven jongens en bij één op de drie à vier meisjes. In vergelijking tot Nederiandse cijfers uit 1983 is het percentage schoolkinderen met wekelijkse hoofdpijnklachten gestegen met $6 \%$, van $17 \%$ naar $23 \%$. De kenmerken van pijn, zoals de kwaliteit van pijn (snijdend/bonkend/zeurend), de locatie in het hoofd, het tijdstip van aanvang, aankondigingsignalen of begeleidende verschijnselen verschillen voor kinderen met veel en kinderen met weinig hoofdpijn. Kinderen met de meeste hoofdpijnklachten scoorden het laagst op kwaliteit van leven en kwaliteit van 
gezondheid. Meer hoofdpijn betekent dat kinderen meer sociale steun zoeken en meer catastroferen en externalizeren en dat ze minder informatie zoeken en minder afleidingstechnieken gebruiken.

Hoofdstuk 5 vergelijkt binnen een kleine groep kinderen $(n=181)$ of de rapportage van hoofdpijnklachten in de vragenlijst van het dwarsdoorsnedeonderzoek overéénkomt met wat deze kinderen daarna invullen als ze, in het kader van de interventiestudie, vier weken lang een hoofdpijndagboek bijhouden. De intensiteit en de duur van hoofdpijn blijkt volgens het dagboek lager te zijn dan aangegeven op de vragenlijst. Op groepsniveau komt de inschatting van de frequentie van hoofdpijn redelijk overéén, maar de overéénstemming op individueel niveau (de correlatie) is laag.

Hoofdstuk 6 en hoof dstuk 7 beschrijven de resultaten van de gerandomiseerde studie naar het effect van de training "Help Jezelf". Deelnemers ( $n=158)$ zijn geselecteerd op basis van de vragenlijst uit het dwarsdoorsnede-onderzoek. Het toeval bepaalde de toewijzing aan de training "Help Jezelf" of aan de placebocontrole groep. De voormeting van de frequentie, de gemiddelde intensiteit en de duur van hoofdpijn werd vergeleken met een nameting direct na de training en na 3, 6 en 12 maanden. Gemiddeld daalde het aantal keren hoofdpijn in 4 weken van 11 keer naar 3 keer. Er bestond geen verschil in effect tussen de "Help Jezelf" groep en de controlegroep, ook niet wat betreft kwaliteit van leven en pijn coping.

Hoofdstuk 8 beschouwt de methodiek en de resultaten van beide studies, aan de hand van een aantal kritische vragen. 


\section{DANKWOORD}




\section{DANKWOORD}

It sil heve!

En iedereen die daaraan heeft bijgedragen wil ik op deze plaats bedanken.

Allereerst natuurlijk de projectleider, mijn promotor, Huda Huijer Abu-Saad. Huda, tijdens mijn studie Verplegingswetenschap vroeg je of ik studentassistent wilde worden. Mijn opdracht was een literatuurstudie naar hoofdpijn bij kinderen, ten behoeve van het schrijven van een subsidie-voorstel dat je wilde indienen bij het NWO. Dat mijn werkzaamheden zouden leiden tot dit proefschrift kon ik toen nog niet bevroeden. Wie weet, jij wel? Je hebt me veel ruimte gegeven in de aanpak van het project en me alle kansen geboden om verder te groeien in de wetenschap. Dat waardeer ik zeer.

Paul Knipschild, tweede promotor, tegenhanger. Bedankt voor je kritische begeleiding. Ik heb veel van je geleerd, vooral toen de artikelen over de interventiestudie geschreven moesten worden.

De leden van de begeleidingscommissie ben ik dankbaar voor hun inzet en betrokkenheid. Jan Passchier, expert op het terrein, tactvol, open in je feedback. Carla Frederiks, methodologisch en persoonlijk een kei. En Frans Feron, onmisbaar. Niet alleen vanwege de medewerking van de afdeling Jeugdgezondheidszorg van de GGD Zuidelijk Zuid-Limburg, maar ook vanwege je enthousiasme voor onderzoek en je vermogen om energie te geven.

Dank ook aan alle mensen die direct of indirect geholpen hebben bij het uitvoeren van het onderzoek. De onderzoeksassistenten die bij het project betrokken waren, eerst Jolanda van Haastregt, daarna Annemarie Rotteveel. Meiden bedankt voor jullie hulp én de gezelligheid. Ook Mandy van den Brink wil ik bedanken. Jij hebt in het kader van je afstudeeronderzoek heel veel werk verzet voor het project en, het tekent je talent en ambitie, er een publicatie uitgesleept. Ook wil ik graag de mensen bedanken die hebben meegedacht bij de vertaling en aanpassing van de interventie of de meetinstrumenten: Tera Boelenvan der Loo, Jeroen Peters en Hans Langeveld. Martijn Berger, hoogleraar Methodologie en statistiek, wil ik danken voor de hulp bij statistische vragen.

Natuurlijk wil ik ook de deelnemers aan het onderzoek niet vergeten: de scholen die zo gastvrij waren om ons te ontvangen, de kinderen die de vragenlijst in de klas hebben ingevuld en vooral de deelnemers aan de interventiestudie. Bedankt allemaal. 
Dank ook aan mijn collega's bij Verplegingswetenschap en later het Centre for Nursing Research en dan vooral de "jonge garde": buurvrouw Tiny, Afke, professor Hoochiekoochie Jan Hamers, Katinka. Het meest speciaal in "Maastricht" was voor mij Inge Buss en ik vind het fijn dat je mijn paranimf wilt zijn. Samen als AIO/OIO begonnen, samen in de trein naar Brabant, samen naar de sauna en vooral samen zwanger.

Andere collega's die ik wil danken zijn Anja van den Hout, Ellen Sebregts, Dianne Alewijnse en Helga Nauta. Ons intervisie-clubje bestond uit lekker eten, latgenotencontact en daarna een methodologisch onderwerp bespreken of een zelf geschreven artikel van één van ons becommentariëren. Nuttig en gewoon erg leuk.

I would also like to thank Bernie Whitaker, friend from Down Under. We first met at the Pediatric Pain Conference in Philadelphia and always stayed in touch. Amazing that we both finished our thesis at the same time. We would love to come over again or to show you, Shirley. Heather and Lochlainn some more of Holland. Thank you for reading my thesis thoroughly and for correcting my English.

Familie en vrienden wil ik bedanken voor hun medeleven en stimulans. Speciaal papa en mama, met jullie vanzelfsprekendheid van studie en werk (ook als er kinderen zijn) en afmaken waar je aan begint; absolute voorwaarden om tot dit proefschrift te komen. En hulp in de vorm van een weekendje oppassen was, zeker in de laatste fase, onontbeerlijk.

Ook onze vrienden en buren in de straat wil ik niet vergeten. Het is heerlijk om een sociaal vangnet te hebben en (nog veel belangrijker) heel veel gezelligheid. Het was voor mij dan ook niet meer dan logisch om jou, Inge, als paranimf te vragen.

Mijn grootste dank gaat uit naar Piet. Alle fasen van het onderzoek en het proefschrift heb je met me doorstaan. Nu deze bevalling achter de rug is (misschien wel de zwaarste van allemaal) verheug ik me op de komst van onze zoon. We gaan er heerlijk van genieten.

It sil heve! 
CURRICULUM VITAE 
158

Curriculum vitae 


\section{CURRICULUM VITAE}

Inez Bandell-Hoekstra werd geboren op 14 april 1969 in het Friese Joure. Na het behalen van het VWO-diploma aan de Rijksscholengemeenschap (RSG) te Heerenveen (1987), volgde zij de Hogere Beroepsopleiding tot Verpleegkundige (HBO-V) aan de Rijkshogeschool Groningen. Hierna startte zij in 1991 de studie Gezondheidswetenschappen aan de Universiteit Maastricht, met als afstudeerrichting Verplegingswetenschap.

Tijdens haar studie werkte ze als student-assistent bij de vakgroep Medische Sociologie, alwaar ze de dataverzameling verzorgde voor het AIO-project Voedingsgewoonten van moeders met jonge kinderen. Daarna was ze studentassistent bij de vakgroep Verplegingswetenschap, waar ze zich bezighield met een voorbereidende literatuurstudie naar hoofdpijn bij kinderen. Tijdens haar studie specialiseerde zij zich in de epidemiologie. Haar afstudeerstage betrof een gerandomiseerd onderzoek naar het effect van post-operatieve pijnbestrijding door middel van "Patient Controlled Analgesia" (PCA) bij kinderen in het Sophia Kinderziekenhuis te Rotterdam. In 1994 behaalde zij cum laude het doctoraaldiploma Gezondheidswetenschappen.

Vanaf september 1994 werkte de auteur als Onderzoeker in Opleiding (OIO) bij de vakgroep Verplegingswetenschap aan de Universiteit Maastricht. Binnen deze aanstelling werkte zij aan het onderzoek naar hoofdpijn bij kinderen, waarvan dit proefschrift het resultaat is. Vanaf december 1997 werkte ze gedurende anderhalf jaar voor 1 dag per week als onderzoeker bij de sectie Verplegingswetenschap, dat behoort tot het cluster Huisarts-, Sociale- en Verpleeghuisgeneeskunde, aan de Katholieke Universiteit Nijmegen. Vanaf oktober 1999 tot september 2001 volgde een aanstelling bij het Centre for Nursing Research van de Universiteit Maastricht, als onderzoeker op het project Stresspreventie bij kinderen op de basisschool. Sinds september 2001 werkt ze als geregistreerd epidemioloog A bij de GGD Hart voor Brabant in 'sHertogenbosch.

Inez is getrouwd met Piet Bandell en heeft samen met hem 2 dochters: Gemma (1998) en Julia (2001). 